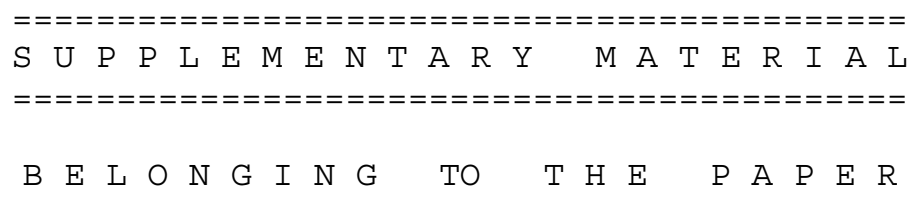

Inorganic Chemistry

b y

\begin{abstract}
Synthesis, Structure, and Catalytic Properties of $\mathrm{V}^{\mathrm{IV}}$, $\mathrm{Mn}^{\mathrm{III}}$, Mo ${ }^{\mathrm{VI}}$ and $\mathrm{U}^{\mathrm{VI}}$ Complexes Containing Bidentate $(\mathrm{N}, \mathrm{O})$ Oxazine and Oxazoline Ligands

Karuppasamy Kandasamy, ${ }^{a}$ Harkesh B. Singh, ${ }^{* a}$ Ray. J. Butcher, ${ }^{b}$ and Jerry P. Jasinskic

a Department of Chemistry, Indian Institute of Technology, Bombay, Powai, Mumbai 400076 India

b Department of Chemistry, Howard University, Washington D.C. 20059, USA

c Department of Chemistry, Keene State College, Keene, NH 03435-2001, USA
\end{abstract}

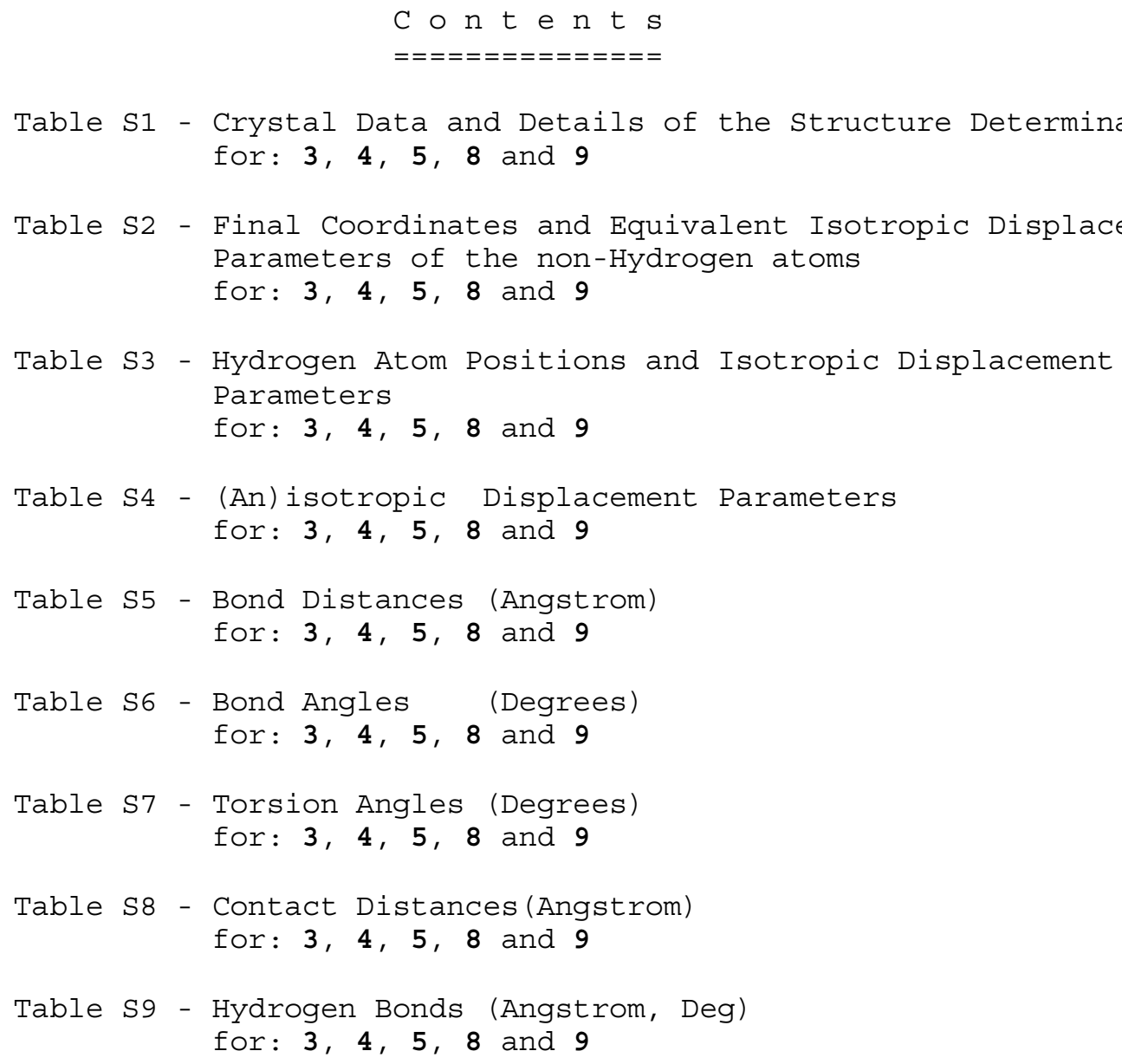


Table S1 - Crystal Data and Details of the Structure Determination for: 3

\section{Crystal Data}

Formula

Formula Weight

Crystal System

Space group

$\mathrm{a}, \mathrm{b}, \mathrm{c}$ [Angstrom]

alpha, beta, gamma [deg]

$\mathrm{V}$ [Ang**3]

Z

$\mathrm{D}(\mathrm{calc}) \quad[\mathrm{g} / \mathrm{cm} * * 3]$

$\mathrm{Mu}(\mathrm{MoKa}) \quad[/ \mathrm{mm}$ ]

$F(000)$

Crystal size [mm]
C10 H11 N O2

$$
177.20
$$

Monoclinic

$\mathrm{P} 21 / \mathrm{n}$

(No. 14)

$9.0577(8) \quad 10.6520(10)$

$9.6107(9)$

$90 \quad 101.457(2)$

$908.79(14)$

4

1.295

0.091

376

$0.00 \times 0.00 \times 0.00$

Data Collection

Temperature (K)

293

Radiation [Angstrom]

MoKa

0.71073

Theta Min-Max [Deg]

$2.9, \quad 28.3$

Dataset

$-12: 12 ;-13: 13 ;-12: 12$

Tot., Uniq. Data, R(int)

$6841, \quad 2203, \quad 0.065$

Observed data [I $>2.0$ sigma(I)]

1253

Refinement

Nref, Npar

$2203, \quad 153$

R， WR2， S

$0.0651,0.1755,1.02$

$\mathrm{w}=1 /\left[\backslash \mathrm{S}^{\wedge} 2^{\wedge}\left(\mathrm{FO}^{\wedge} 2^{\wedge}\right)+(0.0872 \mathrm{P})^{\wedge} 2^{\wedge}+0.0666 \mathrm{P}\right]$ where $\mathrm{P}=\left(\mathrm{FO} \mathrm{O}^{\wedge}{ }^{\wedge}+2 \mathrm{FC}^{\wedge} 2^{\wedge}\right) / 3$

Max. and Av. Shift/Error

$0.01,0.00$

Min. and Max. Resd. Dens. [e/Ang^3]

$-0.16,0.15$ 
Table S2 - Final Coordinates and Equivalent Isotropic Displacement Parameters of the non-Hydrogen atoms

for: 3

\begin{tabular}{|c|c|c|c|c|}
\hline Atom & $\mathrm{X}$ & Y & z & $\mathrm{J}(\mathrm{eq}) \quad\left[\mathrm{Ang}^{\wedge} 2\right]$ \\
\hline---- & --- & --- & --- & ----------- \\
\hline 01 & 0.03508 (19) & $0.74258(16)$ & $1.03185(17)$ & $0.0660(6)$ \\
\hline $\mathrm{O} 2$ & $-0.16904(16)$ & $0.50060(14)$ & $0.70960(16)$ & $0.0654(6)$ \\
\hline N1 & $-0.17878(18)$ & $0.59790(15)$ & $0.92542(18)$ & $0.0507(6)$ \\
\hline $\mathrm{C} 1$ & $0.0951(2)$ & 0.71954 ( 18 ) & $0.9167(2)$ & $0.0470(7)$ \\
\hline $\mathrm{C} 2$ & $0.2276(3)$ & $0.7803(2)$ & $0.9044(3)$ & $0.0615(8)$ \\
\hline $\mathrm{C} 3$ & $0.2941(3)$ & $0.7572(2)$ & $0.7901(3)$ & $0.0713(10)$ \\
\hline $\mathrm{C} 4$ & $0.2297(3)$ & $0.6744(2)$ & $0.6872(3)$ & $0.0707(10)$ \\
\hline C5 & $0.0984(2)$ & $0.6150(2)$ & $0.6965(2)$ & $0.0574(8)$ \\
\hline $\mathrm{C} 6$ & $0.0276(2)$ & $0.63618(17)$ & $0.8112(2)$ & $0.0431(6)$ \\
\hline $\mathrm{C} 7$ & $-0.1162(2)$ & $0.57532(17)$ & $0.8196(2)$ & $0.0437(7)$ \\
\hline * C8B & $-0.2922(7)$ & $0.4171(7)$ & $0.7288(10)$ & $0.060(2)$ \\
\hline * C9B & $-0.3949(9)$ & $0.4779(10)$ & $0.7956(10)$ & $0.142(5)$ \\
\hline * $\mathrm{C} 10 \mathrm{~B}$ & $-0.3253(6)$ & $0.5374(10)$ & $0.9246(9)$ & $0.077(4)$ \\
\hline *C10A & $-0.3289(8)$ & $0.5504(10)$ & $0.9347(10)$ & $0.051(3)$ \\
\hline *C8A & $-0.3270(8)$ & $0.4607(10)$ & $0.6981(13)$ & $0.099(5)$ \\
\hline *C9A & $-0.4050(10)$ & $0.5091(8)$ & $0.7983(9)$ & $0.091(4)$ \\
\hline $\mathrm{U}(\mathrm{eq})$ & $=1 / 3$ of the & trace of the & orthogonalized & U Tensor \\
\hline
\end{tabular}


Table S3 - Hydrogen Atom Positions and Isotropic Displacement Parameters

for: 3

\begin{tabular}{|c|c|c|c|c|c|}
\hline Atom & $\mathrm{x}$ & $\mathrm{Y}$ & $z$ & $\mathrm{U}(\mathrm{iso})$ & {$\left[\mathrm{Ang}^{\wedge} 2\right]$} \\
\hline---- & --- & --- & --- & ----- & ------ \\
\hline $\mathrm{H} 1$ & $-0.052(3)$ & $0.691(3)$ & $1.011(3)$ & & $.097(9)$ \\
\hline $\mathrm{H} 2 \mathrm{~A}$ & 0.27170 & 0.83700 & 0.97390 & & 0.0740 \\
\hline $\mathrm{H} 3 \mathrm{~A}$ & 0.38310 & 0.79810 & 0.78290 & & 0.0850 \\
\hline $\mathrm{H} 4 \mathrm{~A}$ & 0.27550 & 0.65850 & 0.61050 & & 0.0850 \\
\hline *H9BA & -0.46790 & 0.41730 & 0.81550 & & 0.1710 \\
\hline *H9BB & -0.44870 & 0.54050 & 0.73170 & & 0.1710 \\
\hline * $\mathrm{H} 10 \mathrm{C}$ & -0.31160 & 0.47510 & 0.99970 & & 0.0920 \\
\hline$\star_{\mathrm{H} 1 \mathrm{OD}}$ & -0.39400 & 0.60050 & 0.94740 & & 0.0920 \\
\hline *H8BA & -0.34390 & 0.38660 & 0.63690 & & 0.0730 \\
\hline H5A & 0.05530 & 0.55940 & 0.62530 & & 0.0690 \\
\hline *H8BB & -0.25070 & 0.34510 & 0.78520 & & 0.0730 \\
\hline *H8AA & -0.38200 & 0.48370 & 0.60440 & & 0.1200 \\
\hline${ }^{*} \mathrm{H} 8 \mathrm{AB}$ & -0.32860 & 0.36990 & 0.70420 & & 0.1200 \\
\hline *H9AA & -0.47620 & 0.44510 & 0.81390 & & 0.1090 \\
\hline * $\mathrm{H} 9 \mathrm{AB}$ & -0.46400 & 0.57960 & 0.75390 & & 0.1090 \\
\hline *H1 OA & -0.31920 & 0.48110 & 1.00140 & & 0.0610 \\
\hline * $\mathrm{H} 1 \mathrm{OB}$ & -0.38670 & 0.61630 & 0.96850 & & 0.0610 \\
\hline
\end{tabular}

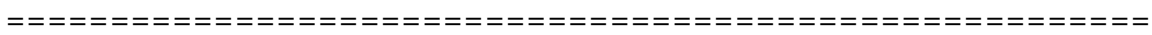

The Temperature Factor has the Form of $\operatorname{Exp}(-\mathrm{T})$ Where $\mathrm{T}=8 *(\mathrm{Pi} * 2) * \mathrm{U} *(\operatorname{Sin}($ Theta $) /$ Lambda $) * * 2$ for Isotropic Atoms 


\begin{tabular}{|c|c|c|c|c|c|c|}
\hline Atom & $\mathrm{U}(1,1)$ or $\mathrm{L}$ & $\mathrm{J} \quad \mathrm{U}(2,2)$ & $\mathrm{U}(3,3)$ & $\mathrm{U}(2,3)$ & $\mathrm{U}(1,3)$ & $\mathrm{U}(1,2)$ \\
\hline-- & ----- & ----- & ----- & ----- & ---- & ----- \\
\hline $\mathrm{O} 1$ & $0.0646(10)$ & $0.0773(12)$ & $0.0593(10)$ & $-0.0195(8)$ & $0.0203(8)$ & $-0.0083(9)$ \\
\hline 2 & $0.0689(10)$ & $0.0745(11)$ & $0.0572(10)$ & $-0.0178(8)$ & $0.0228(8)$ & $-0.0238(8)$ \\
\hline N1 & $0.0472(10)$ & $0.0524(11)$ & $0.0564(11)$ & $-0.0011(8)$ & $0.0199(8)$ & $-0.0003(8)$ \\
\hline 1 & $0.0460(11)$ & $0.0447(12)$ & $0.0513(12)$ & $0.0027(10)$ & $0.0119(9)$ & $0.0056(9)$ \\
\hline 2 & $0.0553(13)$ & $0.0564(14)$ & $0.0722(16)$ & $-0.0008(12)$ & $0.0112(11)$ & $-0.0060(11)$ \\
\hline C3 & $0.0547(13)$ & $0.0696(17)$ & $0.096(2)$ & $0.0117(15)$ & $0.0307(14)$ & $-0.0088(12)$ \\
\hline 4 & $0.0691(15)$ & $0.0786(18)$ & $0.0753(17)$ & $0.0030(14)$ & $0.0408(13)$ & $-0.0050(13)$ \\
\hline C5 & $0.0595(13)$ & $0.0625(14)$ & $0.0549(13)$ & $-0.0015(11)$ & $0.0227(11)$ & $0.0002(11)$ \\
\hline C6 & $0.0436(10)$ & $0.0408(11)$ & $0.0471(11)$ & $0.0059(9)$ & $0.0142(9)$ & $0.0069(8)$ \\
\hline 27 & $0.0473(11)$ & $0.0395(11)$ & $0.0447(12)$ & $0.0036(9)$ & $0.0101(9)$ & $0.0038(8)$ \\
\hline $\mathrm{C} 8 \mathrm{~B}$ & $0.058(3)$ & $0.056(4)$ & $0.065(4)$ & $-0.012(3)$ & $0.007(3)$ & $-0.019(2)$ \\
\hline C9B & $0.072(6)$ & $0.245(11)$ & $0.125(8)$ & $-0.144(8)$ & $0.058(5)$ & $-0.103(6)$ \\
\hline $\mathrm{C} 10 \mathrm{~B}$ & $0.048(6)$ & $0.104(8)$ & $0.083(9)$ & $0.046(6)$ & $0.026(5)$ & $0.033(4)$ \\
\hline $\mathrm{C} 10 \mathrm{~A}$ & $0.050(6)$ & $0.049(4)$ & $0.059(6)$ & $-0.025(4)$ & $0.025(5)$ & $-0.027(4)$ \\
\hline $8 \mathrm{~A}$ & $0.093(6)$ & $0.111(10)$ & $0.097(7)$ & $-0.017(6)$ & $0.025(6)$ & $-0.065(7)$ \\
\hline $9 \mathrm{~A}$ & $0.051(5)$ & $0.099(6)$ & $0.124(9)$ & $0.059(6)$ & $0.023(5)$ & $0.022(4)$ \\
\hline
\end{tabular}

The Temperature Factor has the Form of $\operatorname{Exp}(-T)$ Where $\mathrm{T}=8 *(\mathrm{Pi} * 2) * \mathrm{U} *(\operatorname{Sin}($ Theta $) /$ Lambda $) * * 2$ for Isotropic Atoms $\mathrm{T}=2 *(\mathrm{P} i * 2) * \operatorname{Sumij}(\mathrm{h}(i) * \mathrm{~h}(j) * U(i, j) * A \operatorname{star}(i) * A s t a r(j))$, for Anisotropic Atoms. Astar(i) are Reciprocal Axial Lengths and h(i) are the Reflection Indices. 


\begin{tabular}{|c|c|c|c|c|c|}
\hline $\mathrm{O} 1$ & $-\mathrm{C} 1$ & $1.349(3)$ & $\mathrm{C} 9 \mathrm{~B}$ & $-\mathrm{C} 10 \mathrm{~B}$ & $1.423(13)$ \\
\hline $\mathrm{O} 2$ & $-C 7$ & $1.334(2)$ & $\mathrm{C} 2$ & $-\mathrm{H} 2 \mathrm{~A}$ & 0.9298 \\
\hline $\mathrm{O} 2$ & $-\mathrm{C} 8 \mathrm{~B}$ & $1.467(7)$ & C3 & $-\mathrm{H} 3 \mathrm{~A}$ & 0.9309 \\
\hline $\mathrm{O} 2$ & $-\mathrm{C} 8 \mathrm{~A}$ & $1.475(8)$ & C4 & $-\mathrm{H} 4 \mathrm{~A}$ & 0.9304 \\
\hline $\mathrm{O} 1$ & $-\mathrm{H1}$ & $0.95(3)$ & C5 & $-\mathrm{H} 5 \mathrm{~A}$ & 0.9302 \\
\hline N1 & $-\mathrm{C} 10 \mathrm{~B}$ & $1.474(7)$ & C8A & $-\mathrm{H} 8 \mathrm{AB}$ & 0.9693 \\
\hline N1 & $-\mathrm{C} 10 \mathrm{~A}$ & $1.470(8)$ & C8A & $-\mathrm{H} 8 \mathrm{AA}$ & 0.9700 \\
\hline $\mathrm{N} 1$ & $-\mathrm{C} 7$ & $1.282(3)$ & C8B & $-\mathrm{H} 8 \mathrm{BB}$ & 0.9708 \\
\hline $\mathrm{C} 1$ & $-\mathrm{C} 2$ & $1.389(3)$ & C8B & $-\mathrm{H} 8 \mathrm{BA}$ & 0.9699 \\
\hline $\mathrm{C} 1$ & $-\mathrm{C} 6$ & $1.394(3)$ & C9A & $-\mathrm{H} 9 \mathrm{AB}$ & 0.9701 \\
\hline $\mathrm{C} 2$ & $-\mathrm{C} 3$ & $1.376(4)$ & C9A & $-\mathrm{H} 9 \mathrm{AA}$ & 0.9703 \\
\hline C3 & $-\mathrm{C} 4$ & $1.366(4)$ & C9B & $-\mathrm{H} 9 \mathrm{BB}$ & 0.9697 \\
\hline C4 & $-\mathrm{C5}$ & $1.366(3)$ & C9B & $-\mathrm{H} 9 \mathrm{BA}$ & 0.9699 \\
\hline C5 & $-\mathrm{C} 6$ & $1.399(3)$ & $\mathrm{C} 10 \mathrm{~A}$ & $-\mathrm{H} 10 \mathrm{~B}$ & 0.9695 \\
\hline C6 & $-C 7$ & $1.471(3)$ & C10A & $-\mathrm{H} 10 \mathrm{~A}$ & 0.9701 \\
\hline $\mathrm{C} 8 \mathrm{~A}$ & $-\mathrm{C} 9 \mathrm{~A}$ & $1.401(14)$ & $\mathrm{C} 10 \mathrm{~B}$ & $-\mathrm{H} 10 \mathrm{D}$ & 0.9701 \\
\hline C8B & $-\mathrm{C} 9 \mathrm{~B}$ & $1.391(12)$ & $\mathrm{C} 10 \mathrm{~B}$ & $-\mathrm{H} 10 \mathrm{C}$ & 0.9701 \\
\hline $\mathrm{C} 9 \mathrm{~A}$ & $-\mathrm{C} 10 \mathrm{~A}$ & $1.424(13)$ & & & \\
\hline
\end{tabular}




\begin{tabular}{|c|c|c|c|c|c|c|c|}
\hline Table & $\begin{array}{rl}S 6-B C & f C\end{array}$ & $\begin{array}{l}\text { Angles } \\
3\end{array}$ & (Degrees) & & & & \\
\hline $\mathrm{C} 7$ & -02 & $-C 8 B$ & $115.1(4)$ & $\mathrm{C} 3$ & $-\mathrm{C} 4$ & $-\mathrm{H} 4 \mathrm{~A}$ & 119.83 \\
\hline $\mathrm{C} 7$ & -02 & $-C 8 A$ & $114.9(5)$ & $\mathrm{C} 5$ & $-\mathrm{C} 4$ & $-\mathrm{H} 4 \mathrm{~A}$ & 119.91 \\
\hline $\mathrm{C} 1$ & -01 & $-\mathrm{H} 1$ & $100.1(17)$ & $\mathrm{C} 4$ & $-\mathrm{C} 5$ & $-\mathrm{H} 5 \mathrm{~A}$ & 119.38 \\
\hline C7 & $-\mathrm{N} 1$ & $-\mathrm{C} 10 \mathrm{~A}$ & $122.9(4)$ & $\mathrm{C} 6$ & $-\mathrm{C} 5$ & $-\mathrm{H} 5 \mathrm{~A}$ & 119.41 \\
\hline $\mathrm{C} 7$ & $-\mathrm{N} 1$ & $-\mathrm{C} 10 \mathrm{~B}$ & $117.0(4)$ & $\mathrm{O} 2$ & $-\mathrm{C} 8 \mathrm{~A}$ & $-\mathrm{H} 8 \mathrm{AA}$ & 107.96 \\
\hline $\mathrm{O} 1$ & $-\mathrm{C} 1$ & $-\mathrm{C} 2$ & $118.74(19)$ & $\mathrm{O} 2$ & $-\mathrm{C} 8 \mathrm{~A}$ & $-\mathrm{H} 8 \mathrm{AB}$ & 107.99 \\
\hline $\mathrm{C} 2$ & $-\mathrm{C} 1$ & $-\mathrm{C} 6$ & $119.76(19)$ & C9A & $-\mathrm{C} 8 \mathrm{~A}$ & $-\mathrm{H} 8 \mathrm{AA}$ & 108.00 \\
\hline $\mathrm{O} 1$ & $-\mathrm{Cl}$ & $-\mathrm{C} 6$ & $121.50(17)$ & C9A & $-\mathrm{C} 8 \mathrm{~A}$ & $-\mathrm{H} 8 \mathrm{AB}$ & 108.06 \\
\hline $\mathrm{C} 1$ & $-\mathrm{C} 2$ & $-\mathrm{C} 3$ & $120.5(2)$ & H8AA & $-C 8 A$ & $-\mathrm{H} 8 \mathrm{AB}$ & 107.28 \\
\hline $\mathrm{C} 2$ & $-\mathrm{C} 3$ & $-\mathrm{C} 4$ & $120.1(2)$ & $\mathrm{H} 8 \mathrm{BA}$ & $-C 8 B$ & $-\mathrm{H} 8 \mathrm{BB}$ & 107.89 \\
\hline $\mathrm{C3}$ & $-\mathrm{C} 4$ & $-\mathrm{C} 5$ & $120.3(2)$ & $\mathrm{O} 2$ & $-C 8 B$ & $-\mathrm{H} 8 \mathrm{BB}$ & 109.24 \\
\hline $\mathrm{C} 4$ & $-\mathrm{C} 5$ & $-\mathrm{C} 6$ & $121.2(2)$ & C9B & $-C 8 B$ & $-\mathrm{H} 8 \mathrm{BA}$ & 109.28 \\
\hline $\mathrm{C} 1$ & $-\mathrm{C} 6$ & $-\mathrm{C} 5$ & $118.17(17)$ & $\mathrm{O} 2$ & $-C 8 B$ & $-\mathrm{H} 8 \mathrm{BA}$ & 109.29 \\
\hline $\mathrm{C} 1$ & $-\mathrm{C} 6$ & $-\mathrm{C} 7$ & $120.38(17)$ & C9B & $-C 8 B$ & $-\mathrm{H} 8 \mathrm{BB}$ & 109.30 \\
\hline $\mathrm{C} 5$ & $-\mathrm{C} 6$ & $-\mathrm{C} 7$ & $121.42(17)$ & C8A & $-\mathrm{C} 9 \mathrm{~A}$ & $-\mathrm{H} 9 \mathrm{AB}$ & 106.95 \\
\hline $\mathrm{O} 2$ & $-\mathrm{C} 7$ & $-\mathrm{N} 1$ & $126.53(17)$ & $\mathrm{C} 10 \mathrm{~A}$ & $-\mathrm{C} 9 \mathrm{~A}$ & -H9AA & 106.85 \\
\hline $\mathrm{O} 2$ & $-\mathrm{C} 7$ & $-\mathrm{C} 6$ & $113.68(16)$ & $\mathrm{C} 8 \mathrm{~A}$ & $-\mathrm{C} 9 \mathrm{~A}$ & -H9AA & 106.80 \\
\hline N1 & $-\mathrm{C} 7$ & $-\mathrm{C} 6$ & $119.78(17)$ & $\mathrm{H} 9 \mathrm{AA}$ & $-\mathrm{C} 9 \mathrm{~A}$ & -H9AB & 106.67 \\
\hline $\mathrm{O} 2$ & $-C 8 A$ & $-\mathrm{C} 9 \mathrm{~A}$ & $117.2(8)$ & $\mathrm{C} 10 \mathrm{~A}$ & $-\mathrm{C} 9 \mathrm{~A}$ & $-\mathrm{H} 9 \mathrm{AB}$ & 106.82 \\
\hline $\mathrm{O} 2$ & $-\mathrm{C} 8 \mathrm{~B}$ & $-C 9 B$ & $111.8(7)$ & $\mathrm{C} 8 \mathrm{~B}$ & $-C 9 B$ & -H9BB & 109.02 \\
\hline C8A & $-C 9 A$ & $-\mathrm{C} 10 \mathrm{~A}$ & $121.9(8)$ & C8B & $-C 9 B$ & -H9BA & 109.01 \\
\hline $\mathrm{C} 8 \mathrm{~B}$ & $-C 9 B$ & $-\mathrm{C} 10 \mathrm{~B}$ & $112.9(7)$ & H9BA & $-C 9 B$ & -H9BB & 107.82 \\
\hline $\mathrm{N} 1$ & $-\mathrm{C} 10 \mathrm{~A}$ & $-C 9 A$ & $109.6(7)$ & $\mathrm{C} 10 \mathrm{~B}$ & $-C 9 B$ & -H9BA & 109.02 \\
\hline $\mathrm{N} 1$ & $-\mathrm{C} 10 \mathrm{~B}$ & $-C 9 B$ & $116.2(6)$ & $\mathrm{C} 10 \mathrm{~B}$ & $-C 9 B$ & - H9BB & 108.99 \\
\hline $\mathrm{C} 1$ & $-\mathrm{C} 2$ & $-\mathrm{H} 2 \mathrm{~A}$ & 119.78 & $\mathrm{~N} 1$ & - C10A & $-\mathrm{H} 10 \mathrm{~B}$ & 109.80 \\
\hline $\mathrm{C} 3$ & $-\mathrm{C} 2$ & $-\mathrm{H} 2 \mathrm{~A}$ & 119.74 & $\mathrm{H} 10 \mathrm{~A}$ & $-\mathrm{C} 10 \mathrm{~A}$ & $-\mathrm{H} 10 \mathrm{~B}$ & 108.28 \\
\hline $\mathrm{C} 2$ & $-\mathrm{C3}$ & $-\mathrm{H} 3 \mathrm{~A}$ & 119.90 & C9A & $-\mathrm{C} 10 \mathrm{~A}$ & -H10A & 109.69 \\
\hline
\end{tabular}




$\begin{array}{lll}\text { C4 } & -\mathrm{C} 3 & -\mathrm{H} 3 \mathrm{~A} \\ \text { N1 } & -\mathrm{C} 10 \mathrm{~A} & -\mathrm{H} 10 \mathrm{~A} \\ \mathrm{~N} 1 & -\mathrm{C} 10 \mathrm{~B} & -\mathrm{H} 10 \mathrm{C} \\ \mathrm{N} 1 & -\mathrm{C} 10 \mathrm{~B} & -\mathrm{H} 10 \mathrm{D}\end{array}$

$119.99 \quad \mathrm{C} 9 \mathrm{~A}-\mathrm{C} 10 \mathrm{~A} \quad-\mathrm{H} 10 \mathrm{~B}$

109.71

109.79 $\mathrm{C} 9 \mathrm{~B} \quad-\mathrm{C} 10 \mathrm{~B} \quad-\mathrm{H} 10 \mathrm{C}$

108.21

$108.25 \quad \mathrm{C} 9 \mathrm{~B} \quad-\mathrm{C} 10 \mathrm{~B} \quad-\mathrm{H} 10 \mathrm{D}$

108.20

108.25

H10C

$-\mathrm{C} 10 \mathrm{~B} \quad-\mathrm{H} 10 \mathrm{D}$

107.39 


\begin{tabular}{|c|c|c|c|c|}
\hline $\mathrm{C} 8 \mathrm{~B}$ & -02 & $-\mathrm{C} 7$ & $-\mathrm{N} 1$ & $-14.0(4)$ \\
\hline $\mathrm{C} 8 \mathrm{~B}$ & -02 & $-C 7$ & $-\mathrm{C} 6$ & $166.8(3)$ \\
\hline C7 & -02 & $-C 8 B$ & $-C 9 B$ & $41.1(8)$ \\
\hline $\mathrm{C} 10 \mathrm{~B}$ & $-\mathrm{N} 1$ & $-\mathrm{C} 7$ & -02 & $-1.3(5)$ \\
\hline C7 & $-\mathrm{N} 1$ & $-\mathrm{C} 10 \mathrm{~B}$ & $-C 9 B$ & $-10.6(9)$ \\
\hline $\mathrm{C} 10 \mathrm{~B}$ & $-\mathrm{N} 1$ & $-\mathrm{C7}$ & $-\mathrm{C} 6$ & $177.9(4)$ \\
\hline $\mathrm{C} 6$ & $-\mathrm{C} 1$ & $-\mathrm{C} 2$ & $-\mathrm{C} 3$ & $-1.1(3)$ \\
\hline $\mathrm{O} 1$ & $-\mathrm{Cl}$ & $-\mathrm{C} 2$ & $-\mathrm{C} 3$ & $178.6(2)$ \\
\hline 01 & $-\mathrm{C} 1$ & $-\mathrm{C} 6$ & $-C 7$ & $3.0(3)$ \\
\hline $\mathrm{C} 2$ & $-\mathrm{Cl}$ & $-\mathrm{C} 6$ & $-\mathrm{C} 5$ & $1.1(3)$ \\
\hline O1 & $-\mathrm{C} 1$ & $-\mathrm{C} 6$ & $-\mathrm{C} 5$ & $-178.58(18)$ \\
\hline $\mathrm{C} 2$ & $-\mathrm{C} 1$ & $-\mathrm{C} 6$ & $-C 7$ & $-177.32(19)$ \\
\hline $\mathrm{C} 1$ & $-\mathrm{C} 2$ & $-\mathrm{C} 3$ & $-\mathrm{C} 4$ & $0.3(4)$ \\
\hline $\mathrm{C} 2$ & $-\mathrm{C} 3$ & $-\mathrm{C} 4$ & $-\mathrm{C} 5$ & $0.6(4)$ \\
\hline C3 & $-\mathrm{C} 4$ & $-\mathrm{C} 5$ & $-\mathrm{C} 6$ & $-0.6(3)$ \\
\hline $\mathrm{C} 4$ & $-\mathrm{C} 5$ & $-\mathrm{C} 6$ & $-\mathrm{C} 1$ & $-0.3(3)$ \\
\hline $\mathrm{C} 4$ & $-\mathrm{C} 5$ & $-\mathrm{C} 6$ & $-\mathrm{C} 7$ & $178.2(2)$ \\
\hline $\mathrm{C} 1$ & $-\mathrm{C} 6$ & $-\mathrm{C7}$ & $-\mathrm{N} 1$ & $-0.9(3)$ \\
\hline $\mathrm{C} 1$ & $-\mathrm{C} 6$ & $-\mathrm{C7}$ & -02 & $178.39(17)$ \\
\hline C5 & $-\mathrm{C} 6$ & $-\mathrm{C} 7$ & -02 & $0.0(3)$ \\
\hline C5 & $-\mathrm{C} 6$ & $-\mathrm{C} 7$ & $-\mathrm{N} 1$ & $-179.23(18)$ \\
\hline $\mathrm{O} 2$ & $-C 8 B$ & $-C 9 B$ & $-\mathrm{C} 10 \mathrm{~B}$ & $-52.5(11)$ \\
\hline $\mathrm{C} 8 \mathrm{~B}$ & $-C 9 B$ & $-\mathrm{C} 10 \mathrm{~B}$ & $-\mathrm{N} 1$ & $38.2(12)$ \\
\hline
\end{tabular}


Table s8 - Contact Distances (Angstrom)
for: 3

\begin{tabular}{|c|c|c|c|c|c|}
\hline $\mathrm{O} 1$ & . N1 & $2.527(2)$ & $\mathrm{C} 7$ & . H9BB & 2.9851 \\
\hline $\mathrm{O} 1$ & . C8B_b & $3.388(8)$ & $\mathrm{C} 7$ & . $\mathrm{H} 9 \mathrm{AB}$ & 3.0878 \\
\hline $\mathrm{O} 1$ & .H8BA_a & 2.6204 & $\mathrm{C} 7$ & . $\mathrm{H} 1$ & $2.19(3)$ \\
\hline $\mathrm{O} 1$ & . H8BB_b & 2.5326 & C10A &. $\mathrm{H} 1$ & $2.89(3)$ \\
\hline $\mathrm{O} 1$ & . H4A_C & 2.8135 & $\mathrm{C} 10 \mathrm{~B}$ &. $\mathrm{H} 1$ & $2.95(3)$ \\
\hline $\mathrm{O} 1$ & .H9AB_d & 2.8522 & $\mathrm{H} 1$ &. $\mathrm{~N} 1$ & $1.61(3)$ \\
\hline $\mathrm{O} 2$ & . H5A & 2.4149 & $\mathrm{H} 1$ & . C10A & $2.89(3)$ \\
\hline $\mathrm{O} 2$ & . H2A_e & 2.8158 & $\mathrm{H} 1$ &. $\mathrm{C} 7$ & $2.19(3)$ \\
\hline $\mathrm{N} 1$ & .01 & $2.527(2)$ & $\mathrm{H} 1$ &. $\mathrm{C} 10 \mathrm{~B}$ & $2.95(3)$ \\
\hline $\mathrm{N} 1$ & . $\mathrm{H1}$ & $1.61(3)$ & $\mathrm{H} 1$ & .H4A_C & 2.5533 \\
\hline $\mathrm{C} 1$ & . $C 8 B_{-} a$ & $3.520(8)$ & $\mathrm{H} 1$ & .H8BA_a & 2.5919 \\
\hline $\mathrm{C} 1$ & . C10B_b & $3.592(9)$ & H8AB & . C6_g & 3.0599 \\
\hline $\mathrm{C} 1$ & . C8A_a & $3.557(10)$ & $\mathrm{H} 8 \mathrm{AB}$ & . C1_g & 2.9373 \\
\hline C8A & . C1_g & $3.557(10)$ & $\mathrm{H} 2 \mathrm{~A}$ & $.02 \_d$ & 2.8158 \\
\hline C8B & . C1_g & $3.520(8)$ & H9AB & . C3_i & 2.9671 \\
\hline $\mathrm{C} 8 \mathrm{~B}$ & $.01 \_b$ & $3.388(8)$ & H9AB & . C4_i & 2.9019 \\
\hline $\mathrm{C} 10 \mathrm{~B}$ & . C1_b & $3.592(9)$ & H9AB &. $\mathrm{C} 7$ & 3.0878 \\
\hline $\mathrm{C} 1$ & .H8BA_a & 2.8537 & H9AB & .01 e & 2.8522 \\
\hline $\mathrm{C} 1$ & .H8AB_a & 2.9373 & $\mathrm{H} 4 \mathrm{~A}$ & .01 h & 2.8135 \\
\hline $\mathrm{C} 1$ & . H10C_b & 2.8572 & $\mathrm{H} 4 \mathrm{~A}$ & . $\mathrm{H}_{1} \mathrm{~h}$ & 2.5533 \\
\hline $\mathrm{C} 1$ & .H10A_b & 2.9450 & H8BA & $.01 \_9$ & 2.6204 \\
\hline $\mathrm{C} 1$ & . H8BB_b & 3.0107 & H8BA & . C1_g & 2.8537 \\
\hline $\mathrm{C} 2$ & . H10C_b & 2.9242 & H8BA & .H1_g & 2.5919 \\
\hline $\mathrm{C} 2$ & .H10A_b & 2.9938 & H5A & .02 & 2.4149 \\
\hline $\mathrm{C} 3$ & .H9AB_f & 2.9671 & H8BB & . $\mathrm{Cl}_{1} \mathrm{~b}$ & 3.0107 \\
\hline $\mathrm{C} 4$ & .H9AB_f & 2.9019 & H8BB & .01 b & 2.5326 \\
\hline C6 & .H8AB_a & 3.0599 & H9BB &. $\mathrm{C} 7$ & 2.9851 \\
\hline
\end{tabular}


11

\begin{tabular}{|c|c|c|c|c|c|}
\hline $\mathrm{C} 6$ &. $\mathrm{H} 10 \mathrm{C} \_\mathrm{b}$ & 3.0770 & $\mathrm{H} 10 \mathrm{~A}$ & . $\mathrm{C} 1 \_\mathrm{b}$ & 2.9450 \\
\hline $\mathrm{H} 10 \mathrm{~A}$ & . $\mathrm{C} 2$ _ b & 2.9938 & $\mathrm{H} 10 \mathrm{C}$ & . $C 2 \_b$ & 2.9242 \\
\hline $\mathrm{H} 10 \mathrm{C}$ & . $C 1 \_b$ & 2.8572 & $\mathrm{H} 10 \mathrm{C}$ &. $\mathrm{C} 6 \_\mathrm{b}$ & 3.0770 \\
\hline
\end{tabular}




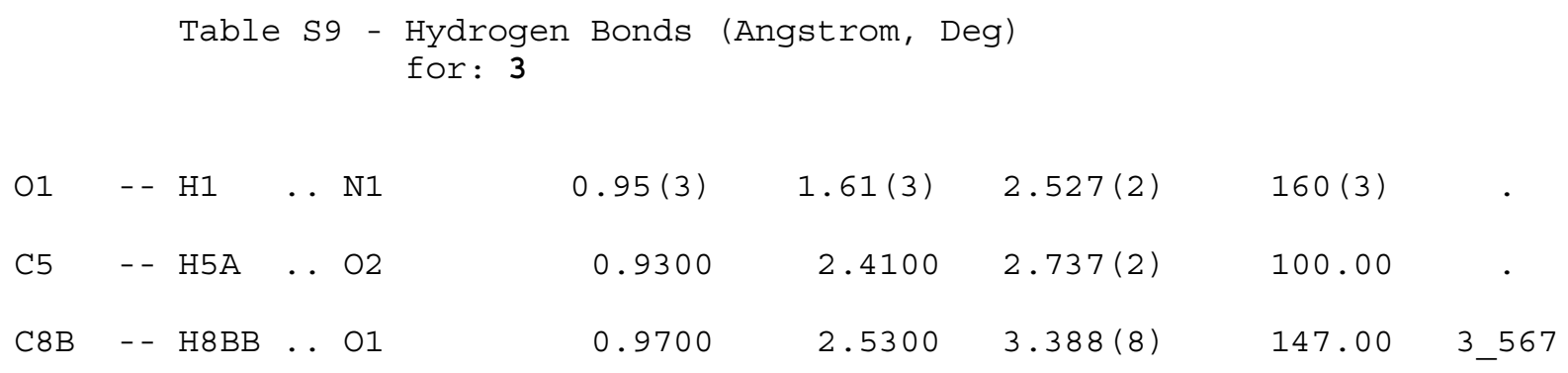


Translation of Symmetry Code to Equiv.Pos

$\mathrm{a}=\left[\begin{array}{ll}2456.00\end{array}\right]=-1 / 2-\mathrm{x}, 1 / 2+\mathrm{y}, 3 / 2-\mathrm{z}$
$\mathrm{b}=\left[\begin{array}{ll}3567.00\end{array}\right]=-\mathrm{x}, 1-\mathrm{y}, 2-\mathrm{z}$
$\mathrm{c}=\left[\begin{array}{ll}4465.00\end{array}\right]=-1 / 2+\mathrm{x}, 3 / 2-\mathrm{y}, 1 / 2+\mathrm{z}$
$\mathrm{d}=\left[\begin{array}{ll}4565.00\end{array}\right]=1 / 2+\mathrm{x}, 3 / 2-\mathrm{y}, 1 / 2+\mathrm{z}$
$\mathrm{e}=\left[\begin{array}{ll}4464.00\end{array}\right]=-1 / 2+\mathrm{x}, 3 / 2-\mathrm{y},-1 / 2+\mathrm{z}$
$\mathrm{f}=\left[\begin{array}{ll}1655.00\end{array}\right]=1+\mathrm{x}, \mathrm{y}, \mathrm{z}$
$\mathrm{g}=\left[\begin{array}{ll}2446.00\end{array}\right]=-1 / 2-\mathrm{x},-1 / 2+\mathrm{y}, 3 / 2-\mathrm{z}$
$\mathrm{h}=\left[\begin{array}{ll}4564.00\end{array}\right]=1 / 2+\mathrm{x}, 3 / 2-\mathrm{y},-1 / 2+\mathrm{z}$
$\mathrm{i}=\left[\begin{array}{ll}1455.00\end{array}\right]=-1+\mathrm{x}, \mathrm{y}, \mathrm{z}$


Table S1 - Crystal Data and Details of the Structure Determination
for: 4 Crystal Data

Formula

Formula Weight

Crystal system

Space group

$\mathrm{a}, \mathrm{b}, \mathrm{c}$ [Angstrom]

alpha, beta, gamma [deg]

$\mathrm{V}$ [Ang**3]

Z

$\mathrm{D}(\mathrm{calc}) \quad[\mathrm{g} / \mathrm{cm} * \star 3]$

$\mathrm{Mu}(\mathrm{MoKa}) \quad$ [ / mm ]

$F(000)$

Crystal size [mm]
C2 2 H24 N2 O5 V

447.37

Monoclinic

P21/C

(No. 14)

$11.8441(6)$

$9.2827(5) \quad 19.8234(10)$

$90 \quad 93.0430(10)$

90

$2176.42(19)$

4

1.365

0.491

932

$0.68 \times 0.54 \times 0.25$

Data Collection

Temperature ( $\mathrm{K})$

Radiation [Angstrom]

MoKa

0.71073

Theta Min-Max [Deg]

$1.7,28.3$

Dataset

$-15: 14 ;-12: 12 ;-26: 25$

Tot., Uniq. Data, R(int)

15971,

5306,

0.038

Observed data [I $>2.0$ sigma(I)]

4107

Refinement

Nref, Npar

$5306, \quad 299$

R， WR2， S

$0.0359,0.0941,1.01$

$\mathrm{W}=1 /\left[\backslash \mathrm{S}^{\wedge} 2^{\wedge}\left(\mathrm{FO} \mathrm{O}^{\wedge} 2^{\wedge}\right)+(0.0525 \mathrm{P})^{\wedge} 2^{\wedge}\right]$

where $\mathrm{P}=\left(\mathrm{FO} \mathrm{O}^{\wedge} 2^{\wedge}+2 \mathrm{FC}^{\wedge} 2^{\wedge}\right) / 3$

Max. and Av. Shift/Error

$0.00,0.00$

Min. and Max. Resd. Dens. [e/Ang^3]

$-0.38,0.41$ 
Table S2 - Final Coordinates and Equivalent Isotropic Displacement Parameters of the non-Hydrogen atoms

for: 4

\begin{tabular}{|c|c|c|c|c|}
\hline Atom & $\mathrm{x}$ & Y & $\mathrm{z}$ & $\mathrm{U}(\mathrm{eq}) \quad\left[\mathrm{Ang}^{\wedge} 2\right]$ \\
\hline--- & --- & --- & -- & - - - - - - - - \\
\hline V & $0.78835(2)$ & $0.37121(3)$ & $0.15667(1)$ & $0.0126(1)$ \\
\hline 0 & $0.70120(10)$ & $0.46621(13)$ & $0.11175(6)$ & $0.0210(4)$ \\
\hline O1A & $0.72170(10)$ & $0.20915(12)$ & $0.19944(6)$ & $0.0179(4)$ \\
\hline O1B & $0.94159(10)$ & $0.43595(12)$ & $0.16138(6)$ & $0.0170(3)$ \\
\hline $\mathrm{O} 2 \mathrm{~A}$ & $0.68628(10)$ & $0.53756(12)$ & $0.34412(5)$ & $0.0165(3)$ \\
\hline $\mathrm{O} 2 \mathrm{~B}$ & $0.93702(10)$ & $0.16665(13)$ & $-0.00969(6)$ & $0.0193(4)$ \\
\hline N1A & $0.77050(11)$ & $0.47861(14)$ & $0.24885(7)$ & $0.0135(4)$ \\
\hline N1B & $0.83814(11)$ & $0.23437(14)$ & $0.07887(7)$ & $0.0142(4)$ \\
\hline $\mathrm{C} 1 \mathrm{~A}$ & $0.65303(14)$ & $0.19792(18)$ & $0.25012(8)$ & $0.0161(5)$ \\
\hline $\mathrm{C} 1 \mathrm{~B}$ & $1.02422(14)$ & $0.43520(17)$ & $0.11890(8)$ & $0.0146(5)$ \\
\hline $\mathrm{C} 2 \mathrm{~A}$ & $0.59433(15)$ & $0.06724(19)$ & $0.25844(9)$ & $0.0194(5)$ \\
\hline $\mathrm{C} 2 \mathrm{~B}$ & $1.11970(14)$ & $0.52228(18)$ & $0.13342(9)$ & $0.0187(5)$ \\
\hline C3A & $0.52078(15)$ & $0.05135(19)$ & $0.30937(9)$ & $0.0200(5)$ \\
\hline C3B & $1.20799(15)$ & $0.52453(18)$ & $0.09050(9)$ & $0.0203(5)$ \\
\hline $\mathrm{C} 4 \mathrm{~A}$ & $0.50192(14)$ & $0.16327(19)$ & $0.35431(9)$ & $0.0199(5)$ \\
\hline C4B & $1.20437(15)$ & $0.44209(19)$ & $0.03194(9)$ & $0.0210(5)$ \\
\hline $\mathrm{C} 5 \mathrm{~A}$ & $0.55913(14)$ & $0.29099(18)$ & $0.34798(8)$ & $0.0169(5)$ \\
\hline C5B & $1.11248(15)$ & $0.35592(18)$ & $0.01672(8)$ & $0.0183(5)$ \\
\hline C6A & $0.63628(13)$ & $0.30918(17)$ & $0.29683(8)$ & $0.0141(5)$ \\
\hline $\mathrm{C} 6 \mathrm{~B}$ & $1.02188(14)$ & $0.34940(17)$ & $0.06015(8)$ & $0.0149(5)$ \\
\hline C7A & $0.70002(14)$ & $0.44237(17)$ & $0.29362(8)$ & $0.0135(5)$ \\
\hline C7B & $0.92791(14)$ & $0.25163(17)$ & $0.04501(8)$ & $0.0148(5)$ \\
\hline $\mathrm{C} 8 \mathrm{~A}$ & $0.74268(15)$ & $0.66902(18)$ & $0.32397(9)$ & $0.0188(5)$ \\
\hline C8B & $0.82712(16)$ & $0.09790(19)$ & $-0.02111(9)$ & $0.0213(5)$ \\
\hline & 447 & 47 & 215 & $0.0211(5)$ \\
\hline
\end{tabular}




$\begin{array}{llccc}\text { C9B } & 0.79114(16) & -0.02219(18) & 0.09003(9) & 0.0222(6) \\ \text { C10A } & 0.82577(14) & 0.61698(17) & 0.27218(8) & 0.0159(5) \\ \text { C10B } & 0.77151(15) & 0.11201(17) & 0.04696(8) & 0.0171(5) \\ \text { C11A } & 0.94124(15) & 0.57997(19) & 0.30580(9) & 0.0199(5) \\ \text { C11B } & 0.64615(15) & 0.1491(2) & 0.03772(9) & 0.0241(6) \\ & & 1 / 3 \text { of the trace of the orthogonalized U Tensor }\end{array}$


Table S3 - Hydrogen Atom Positions and Isotropic Displacement Parameters

for: 4

\begin{tabular}{|c|c|c|c|c|}
\hline Atom & $\mathrm{x}$ & Y & $z$ & $\mathrm{U}($ iso $) \quad\left[\mathrm{Ang}^{\wedge} 2\right]$ \\
\hline--- & --- & --- & -- & --------- \\
\hline $\mathrm{H} 2 \mathrm{AA}$ & 0.60580 & -0.01070 & 0.22850 & $0.032(6)$ \\
\hline $\mathrm{H} 3 \mathrm{AA}$ & 0.48210 & -0.03750 & 0.31400 & $0.018(5)$ \\
\hline $\mathrm{H} 4 \mathrm{AA}$ & 0.45020 & 0.15140 & 0.38890 & $0.030(6)$ \\
\hline H5AA & 0.54660 & 0.36780 & 0.37840 & $0.015(5)$ \\
\hline $\mathrm{H} 8 \mathrm{AA}$ & 0.78340 & 0.71520 & 0.36320 & $0.023(5)$ \\
\hline $\mathrm{H} 8 \mathrm{AB}$ & 0.68770 & 0.73850 & 0.30330 & $0.018(5)$ \\
\hline $\mathrm{H} 2 \mathrm{BA}$ & 1.12350 & 0.58000 & 0.17300 & $0.017(5)$ \\
\hline $\mathrm{H} 3 \mathrm{BA}$ & 1.27210 & 0.58330 & 0.10120 & $0.024(5)$ \\
\hline $\mathrm{H} 4 \mathrm{BA}$ & 1.26520 & 0.44540 & 0.00260 & $0.034(6)$ \\
\hline $\mathrm{H} 9 \mathrm{~A}$ & 0.88920 & 0.69000 & 0.18340 & $0.029(5)$ \\
\hline H9B & 0.85950 & 0.81800 & 0.23360 & $0.032(6)$ \\
\hline H9C & 0.76030 & 0.73580 & 0.19130 & $0.031(6)$ \\
\hline H9D & 0.76830 & -0.00330 & 0.13600 & $0.019(5)$ \\
\hline $\mathrm{H} 9 \mathrm{E}$ & 0.87150 & -0.04780 & 0.09140 & $0.028(5)$ \\
\hline $\mathrm{H} 9 \mathrm{~F}$ & 0.74630 & -0.10200 & 0.07040 & $0.025(5)$ \\
\hline H5BA & 1.10970 & 0.30000 & -0.02350 & $0.027(5)$ \\
\hline H8BA & 0.83580 & -0.00460 & -0.03360 & $0.024(5)$ \\
\hline $\mathrm{H} 11 \mathrm{~A}$ & 0.98630 & 0.52790 & 0.27370 & $0.022(5)$ \\
\hline $\mathrm{H} 11 \mathrm{~B}$ & 0.93100 & 0.51940 & 0.34550 & $0.028(5)$ \\
\hline $\mathrm{H} 11 \mathrm{C}$ & 0.98040 & 0.66890 & 0.31980 & $0.019(5)$ \\
\hline $\mathrm{H} 11 \mathrm{D}$ & 0.61400 & 0.15790 & 0.08210 & $0.024(5)$ \\
\hline $\mathrm{H} 11 \mathrm{E}$ & 0.60670 & 0.07260 & 0.01180 & $0.034(6)$ \\
\hline $\mathrm{H} 11 \mathrm{~F}$ & 0.63720 & 0.24060 & 0.01330 & $0.029(5)$ \\
\hline $\mathrm{H} 8 \mathrm{BB}$ & 0.78160 & 0.14750 & -0.05740 & $0.018(5)$ \\
\hline
\end{tabular}

The Temperature Factor has the Form of $\operatorname{Exp}(-\mathrm{T})$ Where

$T=8 *(\mathrm{Pi} * 2) * \mathrm{U} *(\mathrm{Sin}($ Theta $) /$ Lambda $) * * 2$ for Isotropic Atoms 


\begin{tabular}{|c|c|c|c|c|c|c|}
\hline Atom & $\mathrm{U}(1,1)$ or $\mathrm{U}$ & $\mathrm{U} \quad \mathrm{U}(2,2)$ & $\mathrm{U}(3,3)$ & $\mathrm{U}(2,3)$ & $\mathrm{U}(1,3)$ & $\mathrm{U}(1,2)$ \\
\hline--- & ----- & ----- & ----- & ----- & ----- & ----- \\
\hline V & $0.0148(2)$ & $0.0141(1)$ & $0.0091(1)$ & $0.0002(1)$ & $0.0013(1)$ & 0.0007 (1) \\
\hline O & $0.0244(7)$ & $0.0270(7)$ & $0.0116(6)$ & $0.0001(5)$ & $0.0023(5)$ & $0.0106(5)$ \\
\hline $\mathrm{O} 1 \mathrm{~A}$ & $0.0255(7)$ & $0.0167(6)$ & $0.0120(6)$ & $-0.0024(5)$ & $0.0066(5)$ & $-0.0051(5)$ \\
\hline O1B & $0.0165(6)$ & $0.0193(6)$ & $0.0154(6)$ & $-0.0050(5)$ & $0.0040(5)$ & $-0.0021(5)$ \\
\hline $\mathrm{O} 2 \mathrm{~A}$ & $0.0226(6)$ & $0.0164(6)$ & $0.0109(6)$ & $-0.0022(5)$ & $0.0037(5)$ & $-0.0018(5)$ \\
\hline $\mathrm{O} 2 \mathrm{~B}$ & $0.0250(7)$ & $0.0219(6)$ & $0.0114(6)$ & $-0.0054(5)$ & $0.0041(5)$ & $-0.0002(5)$ \\
\hline $\mathrm{N} 1 \mathrm{~A}$ & $0.0163(7)$ & $0.0128(6)$ & $0.0113(7)$ & $-0.0002(5)$ & $0.0000(6)$ & $-0.0014(5)$ \\
\hline $\mathrm{N} 1 \mathrm{~B}$ & $0.0180(7)$ & $0.0143(7)$ & $0.0101(7)$ & $0.0005(5)$ & $-0.0004(6)$ & $0.0003(5)$ \\
\hline $\mathrm{C} 1 \mathrm{~A}$ & $0.0170(8)$ & $0.0191(8)$ & $0.0120(8)$ & $0.0012(7)$ & $-0.0022(7)$ & $-0.0019(6)$ \\
\hline $\mathrm{C} 1 \mathrm{~B}$ & $0.0179(8)$ & $0.0141(8)$ & $0.0120(8)$ & $0.0027(6)$ & $0.0016(7)$ & $0.0023(6)$ \\
\hline $\mathrm{C} 2 \mathrm{~A}$ & $0.0226(9)$ & $0.0198(9)$ & $0.0155(9)$ & $-0.0012(7)$ & $-0.0005(7)$ & $-0.0056(7)$ \\
\hline $\mathrm{C} 2 \mathrm{~B}$ & $0.0214(9)$ & $0.0175(8)$ & $0.0172(9)$ & $-0.0008(7)$ & $0.0008(7)$ & $-0.0004(7)$ \\
\hline C3A & $0.0199(9)$ & $0.0217(9)$ & $0.0182(9)$ & $0.0028(7)$ & $-0.0016(7)$ & $-0.0076(7)$ \\
\hline C3B & $0.0181(9)$ & $0.0203(9)$ & $0.0224(9)$ & $0.0060(7)$ & $0.0001(7)$ & $-0.0027(7)$ \\
\hline $\mathrm{C} 4 \mathrm{~A}$ & $0.0155(9)$ & $0.0275(9)$ & $0.0169(9)$ & $0.0031(7)$ & $0.0024(7)$ & $-0.0036(7)$ \\
\hline $\mathrm{C} 4 \mathrm{~B}$ & $0.0192(9)$ & $0.0261(10)$ & $0.0184(9)$ & $0.0092(7)$ & $0.0071(7)$ & $0.0037(7)$ \\
\hline $\mathrm{C} 5 \mathrm{~A}$ & $0.0179(9)$ & $0.0211(9)$ & $0.0117(8)$ & $-0.0003(7)$ & $0.0006(7)$ & $0.0006(6)$ \\
\hline C5B & $0.0241(9)$ & $0.0199(9)$ & $0.0110(8)$ & $0.0035(7)$ & $0.0030(7)$ & $0.0047(7)$ \\
\hline $\mathrm{C} 6 \mathrm{~A}$ & $0.0143(8)$ & $0.0170(8)$ & $0.0107(8)$ & $0.0020(6)$ & $-0.0014(6)$ & $-0.0005(6)$ \\
\hline $\mathrm{C} 6 \mathrm{~B}$ & $0.0180(8)$ & $0.0160(8)$ & $0.0108(8)$ & $0.0032(6)$ & $0.0013(7)$ & $0.0037(6)$ \\
\hline C7A & $0.0170(8)$ & $0.0145(8)$ & $0.0088(8)$ & $-0.0006(6)$ & $-0.0024(6)$ & $0.0019(6)$ \\
\hline C7B & $0.0202(9)$ & $0.0155(8)$ & $0.0085(8)$ & $0.0013(6)$ & $-0.0015(7)$ & $0.0054(6)$ \\
\hline $\mathrm{C} 8 \mathrm{~A}$ & $0.0256(9)$ & $0.0142(8)$ & $0.0170(9)$ & $-0.0009(7)$ & $0.0054(7)$ & $-0.0026(7)$ \\
\hline $\mathrm{C} 8 \mathrm{~B}$ & $0.0256(10)$ & $0.0234(9)$ & $0.0148(9)$ & $-0.0043(7)$ & $0.0001(8)$ & $-0.0022(7)$ \\
\hline $\mathrm{C} 9 \mathrm{~A}$ & $0.0300(10)$ & $0.0152(8)$ & $0.0185(9)$ & $0.0012(7)$ & $0.0048(8)$ & $-0.0029(7)$ \\
\hline C9 & $0.0331(11)$ & $0.0164(9)$ & $0.0171(9)$ & $-0.0027(7)$ & $0.0013(8)$ & $-0.0025(7)$ \\
\hline
\end{tabular}




\begin{tabular}{|c|c|c|c|c|c|c|}
\hline C10A & $0.0216(9)$ & $0.0132(8)$ & $0.0131(8)$ & $-0.0030(6)$ & $0.0018(7)$ & $-0.0020(6)$ \\
\hline $\mathrm{C} 10 \mathrm{~B}$ & $0.0233(9)$ & $0.0163(8)$ & $0.0116(8)$ & $-0.0046(6)$ & $-0.0007(7)$ & $-0.0023(6)$ \\
\hline $\mathrm{C} 11 \mathrm{~A}$ & $0.0218(9)$ & $0.0220(9)$ & $0.0157(9)$ & $-0.0037(7)$ & $0.0003(7)$ & $-0.0033(7)$ \\
\hline $\mathrm{C} 11 \mathrm{~B}$ & $0.0230(10)$ & $0.0303(10)$ & $0.0185(9)$ & $-0.0051(8)$ & $-0.0026(8)$ & $-0.0033(8)$ \\
\hline
\end{tabular}

The Temperature Factor has the Form of $\operatorname{Exp}(-\mathrm{T})$ Where $\mathrm{T}=8 *(\mathrm{P} i * * 2) * \mathrm{U} *(\mathrm{Sin}($ Theta $) /$ Lambda $) * * 2$ for Isotropic Atoms $\mathrm{T}=2 *(\mathrm{Pi} * 2) * \operatorname{Sumij}(\mathrm{h}(i) * \mathrm{~h}(j) * \mathrm{U}(i, j) * A s t a r(i) * A s t a r(j))$, for Anisotropic Atoms. Astar(i) are Reciprocal Axial Lengths and h(i) are the Reflection Indices. 

Table S5 - Bond Distances (Angstrom)
for: $\mathbf{4}$

\begin{tabular}{|c|c|c|c|c|c|}
\hline V & -0 & $1.5933(12)$ & $\mathrm{C} 6 \mathrm{~B}$ & $-\mathrm{C} 7 \mathrm{~B}$ & $1.455(2)$ \\
\hline V & $-01 \mathrm{~A}$ & $1.9173(12)$ & C8A & $-C 10 A$ & $1.537(2)$ \\
\hline V & $-01 B$ & $1.9095(12)$ & C8B & $-\mathrm{C} 10 \mathrm{~B}$ & $1.538(2)$ \\
\hline V & $-\mathrm{N} 1 \mathrm{~A}$ & $2.1020(14)$ & C9A & $-\mathrm{C} 10 \mathrm{~A}$ & $1.519(2)$ \\
\hline V & $-N 1 B$ & $2.1063(14)$ & C9B & $-C 10 B$ & $1.521(2)$ \\
\hline $\mathrm{O} 1 \mathrm{~A}$ & $-C 1 A$ & $1.330(2)$ & $\mathrm{C} 10 \mathrm{~A}$ & $-C 11 A$ & $1.528(2)$ \\
\hline O1B & $-C 1 B$ & $1.325(2)$ & $\mathrm{C} 10 \mathrm{~B}$ & $-\mathrm{C} 11 \mathrm{~B}$ & $1.526(3)$ \\
\hline O2A & $-C 7 A$ & $1.3516(19)$ & $\mathrm{C} 2 \mathrm{~A}$ & $-\mathrm{H} 2 \mathrm{AA}$ & 0.9501 \\
\hline O2A & $-C 8 A$ & $1.457(2)$ & $\mathrm{C} 2 \mathrm{~B}$ & $-\mathrm{H} 2 \mathrm{BA}$ & 0.9492 \\
\hline $\mathrm{O} 2 \mathrm{~B}$ & $-C 7 B$ & $1.350(2)$ & C3A & $-\mathrm{H} 3 \mathrm{AA}$ & 0.9503 \\
\hline O2B & $-C 8 B$ & $1.457(2)$ & C3B & $-\mathrm{H} 3 \mathrm{BA}$ & 0.9498 \\
\hline $\mathrm{N} 1 \mathrm{~A}$ & $-C 7 A$ & $1.295(2)$ & $\mathrm{C} 4 \mathrm{~A}$ & $-\mathrm{H} 4 \mathrm{AA}$ & 0.9498 \\
\hline $\mathrm{N} 1 \mathrm{~A}$ & $-\mathrm{C} 10 \mathrm{~A}$ & $1.503(2)$ & $\mathrm{C} 4 \mathrm{~B}$ & $-\mathrm{H} 4 \mathrm{BA}$ & 0.9502 \\
\hline N1B & $-\mathrm{C} 7 \mathrm{~B}$ & $1.297(2)$ & C5A & $-\mathrm{H} 5 \mathrm{AA}$ & 0.9505 \\
\hline N1B & $-\mathrm{C} 10 \mathrm{~B}$ & $1.503(2)$ & $\mathrm{C} 5 \mathrm{~B}$ & $-\mathrm{H} 5 \mathrm{BA}$ & 0.9505 \\
\hline $\mathrm{C} 1 \mathrm{~A}$ & $-C 2 A$ & $1.412(2)$ & $\mathrm{C} 8 \mathrm{~A}$ & $-\mathrm{H} 8 \mathrm{AA}$ & 0.9906 \\
\hline $\mathrm{C} 1 \mathrm{~A}$ & $-\mathrm{C} 6 \mathrm{~A}$ & $1.408(2)$ & $\mathrm{C} 8 \mathrm{~A}$ & $-\mathrm{H} 8 \mathrm{AB}$ & 0.9897 \\
\hline $\mathrm{C} 1 \mathrm{~B}$ & $-C 2 B$ & $1.407(2)$ & $\mathrm{C} 8 \mathrm{~B}$ & $-\mathrm{H} 8 \mathrm{BA}$ & 0.9899 \\
\hline $\mathrm{C} 1 \mathrm{~B}$ & $-C 6 B$ & $1.410(2)$ & $\mathrm{C} 8 \mathrm{~B}$ & $-\mathrm{H} 8 \mathrm{BB}$ & 0.9894 \\
\hline $\mathrm{C} 2 \mathrm{~A}$ & $-C 3 A$ & $1.376(3)$ & C9A & $-\mathrm{H} 9 \mathrm{~A}$ & 0.9804 \\
\hline $\mathrm{C} 2 \mathrm{~B}$ & $-C 3 B$ & $1.383(2)$ & C9A & $-\mathrm{H} 9 \mathrm{~B}$ & 0.9801 \\
\hline C3A & $-C 4 A$ & $1.394(3)$ & C9A & $-\mathrm{H} 9 \mathrm{C}$ & 0.9798 \\
\hline C3B & $-C 4 B$ & $1.389(2)$ & $\mathrm{C} 9 \mathrm{~B}$ & $-\mathrm{H} 9 \mathrm{D}$ & 0.9801 \\
\hline $\mathrm{C} 4 \mathrm{~A}$ & $-C 5 A$ & $1.375(2)$ & C9B & $-\mathrm{H} 9 \mathrm{E}$ & 0.9800 \\
\hline C4B & $-C 5 B$ & $1.371(2)$ & $\mathrm{C} 9 \mathrm{~B}$ & $-\mathrm{H} 9 \mathrm{~F}$ & 0.9800 \\
\hline C5A & $-\mathrm{C} 6 \mathrm{~A}$ & $1.411(2)$ & $\mathrm{C} 11 \mathrm{~A}$ & $-\mathrm{H} 11 \mathrm{~A}$ & 0.9795 \\
\hline C5B & $-C 6 B$ & $1.412(2)$ & $\mathrm{C} 11 \mathrm{~A}$ & $-\mathrm{H} 11 \mathrm{~B}$ & 0.9800 \\
\hline
\end{tabular}




$\begin{array}{llcccc}\text { C6A } & -\mathrm{C} 7 \mathrm{~A} & 1.452(2) & \mathrm{C} 11 \mathrm{~A} & -\mathrm{H} 11 \mathrm{C} & 0.9798 \\ \mathrm{C} 11 \mathrm{~B} & -\mathrm{H} 11 \mathrm{D} & 0.9804 & \mathrm{C} 11 \mathrm{~B} & -\mathrm{H} 11 \mathrm{~F} & 0.9806 \\ \mathrm{C} 11 \mathrm{~B} & -\mathrm{H} 11 \mathrm{E} & 0.9804 & & & \end{array}$



Table s6 - Bond Angles (Degrees)
for: 4

\begin{tabular}{|c|c|c|c|c|c|c|c|}
\hline O & $-\mathrm{V}$ & $-01 \mathrm{~A}$ & $114.31(6)$ & $\mathrm{C} 2 \mathrm{~A}$ & $-C 3 A$ & $-\mathrm{C} 4 \mathrm{~A}$ & $121.20(16)$ \\
\hline O & $-\mathrm{V}$ & $-01 B$ & $116.04(6)$ & $\mathrm{C} 2 \mathrm{~B}$ & $-C 3 B$ & $-C 4 B$ & $121.00(16)$ \\
\hline O & $-\mathrm{V}$ & $-\mathrm{N} 1 \mathrm{~A}$ & $97.54(6)$ & C3A & $-\mathrm{C} 4 \mathrm{~A}$ & $-\mathrm{C} 5 \mathrm{~A}$ & $119.20(16)$ \\
\hline O & $-\mathrm{V}$ & $-\mathrm{N} 1 \mathrm{~B}$ & $97.05(6)$ & $\mathrm{C} 3 \mathrm{~B}$ & $-\mathrm{C} 4 \mathrm{~B}$ & $-\mathrm{C} 5 \mathrm{~B}$ & $119.61(16)$ \\
\hline $\mathrm{O} A \mathrm{~A}$ & $-\mathrm{V}$ & $-01 B$ & $129.64(5)$ & $\mathrm{C} 4 \mathrm{~A}$ & $-\mathrm{C} 5 \mathrm{~A}$ & $-\mathrm{C} 6 \mathrm{~A}$ & $120.67(15)$ \\
\hline $\mathrm{O} 1 \mathrm{~A}$ & $-\mathrm{V}$ & $-\mathrm{N} 1 \mathrm{~A}$ & $85.70(5)$ & $\mathrm{C} 4 \mathrm{~B}$ & $-\mathrm{C} 5 \mathrm{~B}$ & $-\mathrm{C} 6 \mathrm{~B}$ & $120.71(15)$ \\
\hline O1A & $-\mathrm{V}$ & $-N 1 B$ & $89.34(5)$ & $\mathrm{C} 1 \mathrm{~A}$ & $-\mathrm{C} 6 \mathrm{~A}$ & $-\mathrm{C} 5 \mathrm{~A}$ & $120.27(15)$ \\
\hline O1B & $-\mathrm{V}$ & $-\mathrm{N} 1 \mathrm{~A}$ & $86.99(5)$ & $\mathrm{C} 1 \mathrm{~A}$ & $-\mathrm{C} 6 \mathrm{~A}$ & $-\mathrm{C} 7 \mathrm{~A}$ & $120.27(14)$ \\
\hline $\mathrm{O} 1 \mathrm{~B}$ & $-\mathrm{V}$ & $-\mathrm{N} 1 \mathrm{~B}$ & $85.60(5)$ & C5A & $-\mathrm{C} 6 \mathrm{~A}$ & $-\mathrm{C} 7 \mathrm{~A}$ & $119.45(14)$ \\
\hline $\mathrm{N} 1 \mathrm{~A}$ & $-\mathrm{V}$ & $-\mathrm{N} 1 \mathrm{~B}$ & $165.36(5)$ & $\mathrm{C} 1 \mathrm{~B}$ & $-\mathrm{C} 6 \mathrm{~B}$ & $-\mathrm{C} 5 \mathrm{~B}$ & $119.81(15)$ \\
\hline $\mathrm{V}$ & $-01 \mathrm{~A}$ & $-\mathrm{C} 1 \mathrm{~A}$ & $132.71(10)$ & $\mathrm{C} 1 \mathrm{~B}$ & $-\mathrm{C} 6 \mathrm{~B}$ & $-\mathrm{C} 7 \mathrm{~B}$ & $120.27(15)$ \\
\hline $\mathrm{V}$ & $-01 B$ & $-\mathrm{C} 1 \mathrm{~B}$ & $134.41(11)$ & $\mathrm{C} 5 \mathrm{~B}$ & $-\mathrm{C} 6 \mathrm{~B}$ & $-C 7 B$ & $119.91(14)$ \\
\hline C7A & $-02 \mathrm{~A}$ & $-\mathrm{C} 8 \mathrm{~A}$ & $105.63(12)$ & $\mathrm{O} 2 \mathrm{~A}$ & $-C 7 A$ & $-\mathrm{N} 1 \mathrm{~A}$ & $116.44(14)$ \\
\hline C7B & $-02 B$ & $-\mathrm{C} 8 \mathrm{~B}$ & $105.78(13)$ & $\mathrm{O} 2 \mathrm{~A}$ & $-C 7 A$ & $-\mathrm{C} 6 \mathrm{~A}$ & $116.17(14)$ \\
\hline $\mathrm{V}$ & $-N 1 A$ & $-C 7 A$ & $124.81(11)$ & N1A & $-C 7 A$ & $-\mathrm{C} 6 \mathrm{~A}$ & $127.37(15)$ \\
\hline V & $-\mathrm{N} 1 \mathrm{~A}$ & $-\mathrm{C} 10 \mathrm{~A}$ & $127.62(10)$ & $\mathrm{O} 2 \mathrm{~B}$ & $-C 7 B$ & $-N 1 B$ & $116.50(14)$ \\
\hline C7A & $-\mathrm{N} 1 \mathrm{~A}$ & $-\mathrm{C} 10 \mathrm{~A}$ & $107.29(13)$ & $\mathrm{O} 2 \mathrm{~B}$ & $-C 7 B$ & $-\mathrm{C} 6 \mathrm{~B}$ & $115.93(14)$ \\
\hline V & $-\mathrm{N} 1 \mathrm{~B}$ & $-C 7 B$ & $124.99(11)$ & $\mathrm{N} 1 \mathrm{~B}$ & $-C 7 B$ & $-\mathrm{C} 6 \mathrm{~B}$ & $127.54(15)$ \\
\hline $\mathrm{V}$ & $-N 1 B$ & $-\mathrm{C} 10 \mathrm{~B}$ & $127.06(10)$ & $\mathrm{O} 2 \mathrm{~A}$ & $-C 8 A$ & $-\mathrm{C10A}$ & $103.83(13)$ \\
\hline $\mathrm{C} 7 \mathrm{~B}$ & $-N 1 B$ & $-\mathrm{C} 10 \mathrm{~B}$ & $107.58(13)$ & $\mathrm{O} 2 \mathrm{~B}$ & $-C 8 B$ & $-\mathrm{C} 10 \mathrm{~B}$ & $104.31(13)$ \\
\hline $\mathrm{O} 1 \mathrm{~A}$ & $-C 1 A$ & $-\mathrm{C} 2 \mathrm{~A}$ & $118.70(15)$ & $\mathrm{N} 1 \mathrm{~A}$ & $-\mathrm{C} 10 \mathrm{~A}$ & $-\mathrm{C} 8 \mathrm{~A}$ & $100.96(13)$ \\
\hline $\mathrm{O} 1 \mathrm{~A}$ & $-C 1 A$ & $-\mathrm{C} 6 \mathrm{~A}$ & $123.53(15)$ & $\mathrm{N} 1 \mathrm{~A}$ & $-\mathrm{C} 10 \mathrm{~A}$ & $-\mathrm{C} 9 \mathrm{~A}$ & $112.28(13)$ \\
\hline $\mathrm{C} 2 \mathrm{~A}$ & $-C 1 A$ & $-\mathrm{C} 6 \mathrm{~A}$ & $117.76(15)$ & N1A & $-\mathrm{CIOA}$ & $-\mathrm{C} 11 \mathrm{~A}$ & $107.88(13)$ \\
\hline O1B & $-\mathrm{C} 1 \mathrm{~B}$ & $-\mathrm{C} 2 \mathrm{~B}$ & $118.67(14)$ & C8A & $-\mathrm{C} 10 \mathrm{~A}$ & $-\mathrm{C} 9 \mathrm{~A}$ & $111.23(14)$ \\
\hline O1B & $-C 1 B$ & $-\mathrm{C} 6 \mathrm{~B}$ & $123.05(15)$ & $\mathrm{C} 8 \mathrm{~A}$ & $-\mathrm{CIOA}$ & $-\mathrm{C} 11 \mathrm{~A}$ & $111.67(14)$ \\
\hline $\mathrm{C} 2 \mathrm{~B}$ & $-\mathrm{C} 1 \mathrm{~B}$ & $-\mathrm{C} 6 \mathrm{~B}$ & $118.28(15)$ & C9A & $-\mathrm{C} 10 \mathrm{~A}$ & $-\mathrm{C} 11 \mathrm{~A}$ & $112.25(14)$ \\
\hline $\mathrm{C} 1 \mathrm{~A}$ & $-\mathrm{C} 2 \mathrm{~A}$ & $-C 3 A$ & $120.83(16)$ & N1B & $-\mathrm{C} 10 \mathrm{~B}$ & $-C 8 B$ & $101.17(13)$ \\
\hline
\end{tabular}




\begin{tabular}{|c|c|c|c|c|c|c|c|}
\hline $\mathrm{C} 1 \mathrm{~B}$ & $-\mathrm{C} 2 \mathrm{~B}$ & $-C 3 B$ & $120.57(16)$ & $\mathrm{N} 1 \mathrm{~B}$ & $-\mathrm{C} 10 \mathrm{~B}$ & $-\mathrm{C} 9 \mathrm{~B}$ & $108.75(13)$ \\
\hline $\mathrm{N} 1 \mathrm{~B}$ & $-\mathrm{C} 10 \mathrm{~B}$ & $-C 11 B$ & $111.46(13)$ & $\mathrm{C} 10 \mathrm{~B}$ & $-\mathrm{C} 8 \mathrm{~B}$ & $-\mathrm{H} 8 \mathrm{BA}$ & 110.87 \\
\hline $\mathrm{C} 8 \mathrm{~B}$ & $-\mathrm{C} 10 \mathrm{~B}$ & $-C 9 B$ & $111.27(14)$ & $\mathrm{C} 10 \mathrm{~B}$ & $-C 8 B$ & $-\mathrm{H} 8 \mathrm{BB}$ & 110.86 \\
\hline $\mathrm{C} 8 \mathrm{~B}$ & $-\mathrm{C} 10 \mathrm{~B}$ & $-\mathrm{C} 11 \mathrm{~B}$ & $111.91(14)$ & $\mathrm{H} 8 \mathrm{BA}$ & $-C 8 B$ & $-\mathrm{H} 8 \mathrm{BB}$ & 108.98 \\
\hline C9B & $-\mathrm{C} 10 \mathrm{~B}$ & $-C 11 B$ & $111.78(14)$ & C10A & $-C 9 A$ & $-\mathrm{H} 9 \mathrm{~A}$ & 109.50 \\
\hline $\mathrm{C} 1 \mathrm{~A}$ & $-\mathrm{C} 2 \mathrm{~A}$ & $-\mathrm{H} 2 \mathrm{AA}$ & 119.57 & $\mathrm{C} 10 \mathrm{~A}$ & $-C 9 A$ & $-\mathrm{H} 9 \mathrm{~B}$ & 109.46 \\
\hline C3A & $-\mathrm{C} 2 \mathrm{~A}$ & $-\mathrm{H} 2 \mathrm{AA}$ & 119.59 & C10A & $-\mathrm{C} 9 \mathrm{~A}$ & $-\mathrm{H} 9 \mathrm{C}$ & 109.43 \\
\hline $\mathrm{C} 1 \mathrm{~B}$ & $-\mathrm{C} 2 \mathrm{~B}$ & $-\mathrm{H} 2 \mathrm{BA}$ & 119.71 & $\mathrm{H} 9 \mathrm{~A}$ & $-\mathrm{C} 9 \mathrm{~A}$ & $-\mathrm{H} 9 \mathrm{~B}$ & 109.49 \\
\hline $\mathrm{C} 3 \mathrm{~B}$ & $-\mathrm{C} 2 \mathrm{~B}$ & -H2BA & 119.72 & $\mathrm{H} 9 \mathrm{~A}$ & $-\mathrm{C} 9 \mathrm{~A}$ & $-\mathrm{H} 9 \mathrm{C}$ & 109.47 \\
\hline $\mathrm{C} 2 \mathrm{~A}$ & $-C 3 A$ & $-\mathrm{H} 3 \mathrm{AA}$ & 119.42 & H9B & $-\mathrm{C} 9 \mathrm{~A}$ & $-\mathrm{H} 9 \mathrm{C}$ & 109.48 \\
\hline $\mathrm{C} 4 \mathrm{~A}$ & $-C 3 A$ & -H3AA & 119.38 & $\mathrm{C} 10 \mathrm{~B}$ & $-C 9 B$ & -H9D & 109.50 \\
\hline $\mathrm{C} 2 \mathrm{~B}$ & $-C 3 B$ & $-\mathrm{H} 3 \mathrm{BA}$ & 119.54 & $\mathrm{C} 10 \mathrm{~B}$ & $-C 9 B$ & $-\mathrm{H} 9 \mathrm{E}$ & 109.48 \\
\hline $\mathrm{C} 4 \mathrm{~B}$ & $-C 3 B$ & -Н3BA & 119.46 & $\mathrm{C} 10 \mathrm{~B}$ & $-C 9 B$ & $-\mathrm{H} 9 \mathrm{~F}$ & 109.44 \\
\hline C3A & $-C 4 A$ & -H4AA & 120.41 & H9D & $-C 9 B$ & $-\mathrm{H} 9 \mathrm{E}$ & 109.47 \\
\hline C5A & $-C 4 A$ & $-\mathrm{H} 4 \mathrm{AA}$ & 120.39 & H9D & $-C 9 B$ & $-\mathrm{H} 9 \mathrm{~F}$ & 109.51 \\
\hline C3B & $-C 4 B$ & -H4 BA & 120.19 & H9E & $-C 9 B$ & $-\mathrm{H} 9 \mathrm{~F}$ & 109.43 \\
\hline C5B & $-C 4 B$ & -H4BA & 120.19 & $\mathrm{C} 10 \mathrm{~A}$ & $-\mathrm{C} 11 \mathrm{~A}$ & $-\mathrm{H} 11 \mathrm{~A}$ & 109.48 \\
\hline $\mathrm{C} 4 \mathrm{~A}$ & $-C 5 A$ & - H5AA & 119.65 & $\mathrm{C} 10 \mathrm{~A}$ & $-\mathrm{C} 11 \mathrm{~A}$ & $-\mathrm{H} 11 \mathrm{~B}$ & 109.46 \\
\hline C6A & $-\mathrm{C} 5 \mathrm{~A}$ & -H5AA & 119.68 & $\mathrm{C} 10 \mathrm{~A}$ & $-\mathrm{C} 11 \mathrm{~A}$ & $-\mathrm{H} 11 \mathrm{C}$ & 109.47 \\
\hline $\mathrm{C} 4 \mathrm{~B}$ & $-C 5 B$ & -H5BA & 119.66 & $\mathrm{H} 11 \mathrm{~A}$ & $-\mathrm{C} 11 \mathrm{~A}$ & $-\mathrm{H} 11 \mathrm{~B}$ & 109.48 \\
\hline C6B & $-C 5 B$ & -H5BA & 119.63 & $\mathrm{H} 11 \mathrm{~A}$ & $-\mathrm{C} 11 \mathrm{~A}$ & $-\mathrm{H} 11 \mathrm{C}$ & 109.49 \\
\hline $\mathrm{O} 2 \mathrm{~A}$ & $-C 8 A$ & $-\mathrm{H} 8 \mathrm{AA}$ & 111.00 & $\mathrm{H} 11 \mathrm{~B}$ & $-\mathrm{C} 11 \mathrm{~A}$ & $-\mathrm{H} 11 \mathrm{C}$ & 109.44 \\
\hline $\mathrm{O} 2 \mathrm{~A}$ & $-C 8 A$ & $-\mathrm{H} 8 \mathrm{AB}$ & 110.98 & $\mathrm{C} 10 \mathrm{~B}$ & $-\mathrm{C} 11 \mathrm{~B}$ & $-\mathrm{H} 11 \mathrm{D}$ & 109.45 \\
\hline C10A & $-C 8 A$ & -H8AA & 110.98 & $\mathrm{C} 10 \mathrm{~B}$ & $-C 11 B$ & $-\mathrm{H} 11 \mathrm{E}$ & 109.46 \\
\hline $\mathrm{C} 10 \mathrm{~A}$ & $-C 8 A$ & $-\mathrm{H} 8 \mathrm{AB}$ & 111.02 & $\mathrm{C} 10 \mathrm{~B}$ & $-C 11 B$ & $-\mathrm{H} 11 \mathrm{~F}$ & 109.50 \\
\hline H8AA & $-C 8 A$ & $-\mathrm{H} 8 \mathrm{AB}$ & 108.98 & $\mathrm{H} 11 \mathrm{D}$ & $-C 11 B$ & $-\mathrm{H} 11 \mathrm{E}$ & 109.49 \\
\hline $\mathrm{O} 2 \mathrm{~B}$ & $-C 8 B$ & -H8BA & 110.87 & $\mathrm{H} 11 \mathrm{D}$ & $-\mathrm{C} 11 \mathrm{~B}$ & $-\mathrm{H} 11 \mathrm{~F}$ & 109.50 \\
\hline $\mathrm{O} 2 \mathrm{~B}$ & $-C 8 B$ & $-\mathrm{H} 8 \mathrm{BB}$ & 110.92 & $\mathrm{H} 11 \mathrm{E}$ & $-C 11 B$ & $-\mathrm{H} 11 \mathrm{~F}$ & 109.43 \\
\hline
\end{tabular}




\begin{tabular}{|c|c|c|c|c|}
\hline $\mathrm{O}$ & $-\mathrm{V}$ & $-01 A$ & $-C 1 A$ & $-71.03(15)$ \\
\hline $\mathrm{O} 1 \mathrm{~B}$ & $-\mathrm{V}$ & $-01 \mathrm{~A}$ & $-\mathrm{C} 1 \mathrm{~A}$ & $107.80(14)$ \\
\hline $\mathrm{N} 1 \mathrm{~A}$ & $-\mathrm{V}$ & $-01 \mathrm{~A}$ & $-\mathrm{C} 1 \mathrm{~A}$ & $25.31(14)$ \\
\hline $\mathrm{N} 1 \mathrm{~B}$ & $-\mathrm{V}$ & $-01 \mathrm{~A}$ & $-\mathrm{C} 1 \mathrm{~A}$ & $-168.48(14)$ \\
\hline $\mathrm{O}$ & $-\mathrm{V}$ & $-01 B$ & $-\mathrm{C} 1 \mathrm{~B}$ & $-73.62(15)$ \\
\hline $\mathrm{O} 1 \mathrm{~A}$ & $-\mathrm{V}$ & $-01 B$ & $-\mathrm{C} 1 \mathrm{~B}$ & $107.57(15)$ \\
\hline $\mathrm{N} 1 \mathrm{~A}$ & $-\mathrm{V}$ & $-01 B$ & $-\mathrm{C} 1 \mathrm{~B}$ & $-170.55(15)$ \\
\hline $\mathrm{N} 1 \mathrm{~B}$ & $-\mathrm{V}$ & $-01 B$ & $-\mathrm{C} 1 \mathrm{~B}$ & $22.09(14)$ \\
\hline 0 & $-\mathrm{V}$ & $-N 1 A$ & $-C 7 A$ & $92.10(14)$ \\
\hline 0 & $-\mathrm{V}$ & $-\mathrm{N} 1 \mathrm{~A}$ & - C10A & $-81.03(13)$ \\
\hline $\mathrm{O} 1 \mathrm{~A}$ & $-\mathrm{V}$ & $-\mathrm{N} 1 \mathrm{~A}$ & $-C 7 A$ & $-21.88(13)$ \\
\hline $\mathrm{O} 1 \mathrm{~A}$ & $-\mathrm{V}$ & $-\mathrm{N} 1 \mathrm{~A}$ & $-\mathrm{C} 10 \mathrm{~A}$ & $164.99(13)$ \\
\hline O1B & $-\mathrm{V}$ & $-\mathrm{N} 1 \mathrm{~A}$ & $-C 7 A$ & $-152.02(14)$ \\
\hline O1B & $-\mathrm{V}$ & $-\mathrm{N} 1 \mathrm{~A}$ & $-\mathrm{C} 10 \mathrm{~A}$ & $34.85(12)$ \\
\hline 0 & $-\mathrm{V}$ & $-\mathrm{N} 1 \mathrm{~B}$ & $-C 7 B$ & $96.82(14)$ \\
\hline 0 & $-\mathrm{V}$ & $-\mathrm{N} 1 \mathrm{~B}$ & $-\mathrm{C} 10 \mathrm{~B}$ & $-75.30(13)$ \\
\hline $\mathrm{O} 1 \mathrm{~A}$ & $-\mathrm{V}$ & $-\mathrm{N} 1 \mathrm{~B}$ & $-C 7 B$ & $-148.76(14)$ \\
\hline $\mathrm{O} 1 \mathrm{~A}$ & $-\mathrm{V}$ & $-N 1 B$ & $-\mathrm{C} 10 \mathrm{~B}$ & $39.12(13)$ \\
\hline O1B & $-\mathrm{V}$ & $-\mathrm{N} 1 \mathrm{~B}$ & $-\mathrm{C} 7 \mathrm{~B}$ & $-18.91(13)$ \\
\hline O1B & $-\mathrm{V}$ & $-\mathrm{N} 1 \mathrm{~B}$ & $-\mathrm{C} 10 \mathrm{~B}$ & $168.97(13)$ \\
\hline V & $-01 \mathrm{~A}$ & $-\mathrm{C} 1 \mathrm{~A}$ & $-\mathrm{C} 2 \mathrm{~A}$ & $163.10(12)$ \\
\hline V & $-01 \mathrm{~A}$ & $-C 1 A$ & $-\mathrm{C} 6 \mathrm{~A}$ & $-17.6(2)$ \\
\hline V & $-01 B$ & $-\mathrm{C} 1 \mathrm{~B}$ & $-\mathrm{C} 2 \mathrm{~B}$ & $165.36(12)$ \\
\hline V & $-01 B$ & $-\mathrm{C} 1 \mathrm{~B}$ & $-\mathrm{C} 6 \mathrm{~B}$ & $-15.6(2)$ \\
\hline C8A & $-02 \mathrm{~A}$ & $-C 7 A$ & $-N 1 A$ & $10.76(19)$ \\
\hline C8A & $-02 \mathrm{~A}$ & $-C 7 A$ & $-\mathrm{C} 6 \mathrm{~A}$ & $-170.88(14)$ \\
\hline C7A & $-02 \mathrm{~A}$ & $-C 8 A$ & $-\mathrm{C} 10 \mathrm{~A}$ & $-21.33(16)$ \\
\hline $\mathrm{C} 8 \mathrm{~B}$ & $-\mathrm{O} 2 \mathrm{~B}$ & $-\mathrm{C} 7 \mathrm{~B}$ & $-N 1 B$ & $11.00(19)$ \\
\hline
\end{tabular}




\begin{tabular}{|c|c|c|c|c|}
\hline C8B & $-\mathrm{O} 2 \mathrm{~B}$ & $-C 7 B$ & $-\mathrm{C} 6 \mathrm{~B}$ & $-170.65(14)$ \\
\hline C7B & $-02 \mathrm{~B}$ & $-\mathrm{C} 8 \mathrm{~B}$ & $-\mathrm{C} 10 \mathrm{~B}$ & $-19.71(16)$ \\
\hline V & $-\mathrm{N} 1 \mathrm{~A}$ & $-C 7 A$ & $-\mathrm{C} 6 \mathrm{~A}$ & $13.0(2)$ \\
\hline $\mathrm{C} 10 \mathrm{~A}$ & $-\mathrm{N} 1 \mathrm{~A}$ & $-C 7 A$ & $-02 \mathrm{~A}$ & $5.41(19)$ \\
\hline $\mathrm{V}$ & $-\mathrm{N} 1 \mathrm{~A}$ & $-C 7 A$ & $-02 \mathrm{~A}$ & $-168.89(10)$ \\
\hline C7A & $-\mathrm{N} 1 \mathrm{~A}$ & $-\mathrm{C} 10 \mathrm{~A}$ & $-C 8 A$ & $-17.99(16)$ \\
\hline C7A & $-\mathrm{N} 1 \mathrm{~A}$ & $-\mathrm{C} 10 \mathrm{~A}$ & $-\mathrm{C} 9 \mathrm{~A}$ & $-136.55(15)$ \\
\hline C7A & $-\mathrm{N} 1 \mathrm{~A}$ & $-\mathrm{C} 10 \mathrm{~A}$ & $-\mathrm{C} 11 \mathrm{~A}$ & $99.25(15)$ \\
\hline $\mathrm{C} 10 \mathrm{~A}$ & $-\mathrm{N} 1 \mathrm{~A}$ & $-C 7 A$ & $-\mathrm{C} 6 \mathrm{~A}$ & $-172.73(15)$ \\
\hline V & $-\mathrm{N} 1 \mathrm{~A}$ & $-\mathrm{C} 10 \mathrm{~A}$ & $-C 8 A$ & $156.11(11)$ \\
\hline V & $-\mathrm{N} 1 \mathrm{~A}$ & $-\mathrm{C} 10 \mathrm{~A}$ & $-C 9 A$ & $37.55(18)$ \\
\hline V & $-\mathrm{N} 1 \mathrm{~A}$ & $-\mathrm{C} 10 \mathrm{~A}$ & $-\mathrm{C} 11 \mathrm{~A}$ & $-86.66(15)$ \\
\hline $\mathrm{V}$ & $-\mathrm{N} 1 \mathrm{~B}$ & $-\mathrm{C} 10 \mathrm{~B}$ & $-C 8 B$ & $158.11(11)$ \\
\hline V & $-N 1 B$ & $-\mathrm{C} 10 \mathrm{~B}$ & $-C 9 B$ & $-84.65(16)$ \\
\hline $\mathrm{V}$ & $-\mathrm{N} 1 \mathrm{~B}$ & $-\mathrm{C} 10 \mathrm{~B}$ & $-C 11 B$ & $39.01(18)$ \\
\hline C7B & $-N 1 B$ & $-\mathrm{C} 10 \mathrm{~B}$ & $-C 8 B$ & $-15.12(16)$ \\
\hline C7B & $-N 1 B$ & $-\mathrm{C} 10 \mathrm{~B}$ & $-C 9 B$ & $102.12(16)$ \\
\hline C7B & $-N 1 B$ & $-\mathrm{C} 10 \mathrm{~B}$ & $-C 11 B$ & $-134.22(14)$ \\
\hline $\mathrm{C} 10 \mathrm{~B}$ & $-N 1 B$ & $-C 7 B$ & $-02 B$ & $3.33(19)$ \\
\hline $\mathrm{C} 10 \mathrm{~B}$ & $-N 1 B$ & $-\mathrm{C} 7 \mathrm{~B}$ & $-\mathrm{C} 6 \mathrm{~B}$ & $-174.80(15)$ \\
\hline V & $-N 1 B$ & $-C 7 B$ & $-\mathrm{O} 2 \mathrm{~B}$ & $-170.07(10)$ \\
\hline V & $-N 1 B$ & $-C 7 B$ & $-\mathrm{C} 6 \mathrm{~B}$ & $11.8(2)$ \\
\hline $\mathrm{C} 2 \mathrm{~A}$ & $-\mathrm{C} 1 \mathrm{~A}$ & $-\mathrm{C} 6 \mathrm{~A}$ & $-\mathrm{C} 5 \mathrm{~A}$ & $-2.9(2)$ \\
\hline O1A & $-\mathrm{C} 1 \mathrm{~A}$ & $-\mathrm{C} 6 \mathrm{~A}$ & $-\mathrm{C} 5 \mathrm{~A}$ & $177.89(15)$ \\
\hline O1A & $-\mathrm{C} 1 \mathrm{~A}$ & $-\mathrm{C} 6 \mathrm{~A}$ & $-C 7 A$ & $-3.4(2)$ \\
\hline O1A & $-\mathrm{C} 1 \mathrm{~A}$ & $-\mathrm{C} 2 \mathrm{~A}$ & $-C 3 A$ & $-178.81(16)$ \\
\hline $\mathrm{C} 6 \mathrm{~A}$ & $-\mathrm{C} 1 \mathrm{~A}$ & $-\mathrm{C} 2 \mathrm{~A}$ & $-C 3 A$ & $1.9(3)$ \\
\hline $\mathrm{C} 2 \mathrm{~A}$ & $-\mathrm{C} 1 \mathrm{~A}$ & $-C 6 A$ & $-C 7 A$ & $175.84(15)$ \\
\hline O1B & $-\mathrm{C} 1 \mathrm{~B}$ & $-\mathrm{C} 6 \mathrm{~B}$ & $-C 7 B$ & $-2.4(2)$ \\
\hline
\end{tabular}




\begin{tabular}{|c|c|c|c|c|}
\hline $\mathrm{C} 2 \mathrm{~B}$ & $-\mathrm{C} 1 \mathrm{~B}$ & $-\mathrm{C} 6 \mathrm{~B}$ & $-C 5 B$ & $-1.9(2)$ \\
\hline O1B & $-\mathrm{C} 1 \mathrm{~B}$ & $-\mathrm{C} 2 \mathrm{~B}$ & $-C 3 B$ & $179.88(15)$ \\
\hline $\mathrm{C} 6 \mathrm{~B}$ & $-\mathrm{C} 1 \mathrm{~B}$ & $-\mathrm{C} 2 \mathrm{~B}$ & $-C 3 B$ & $0.8(2)$ \\
\hline $\mathrm{C} 2 \mathrm{~B}$ & $-\mathrm{C} 1 \mathrm{~B}$ & $-\mathrm{C} 6 \mathrm{~B}$ & $-C 7 B$ & $176.56(15)$ \\
\hline O1B & $-\mathrm{C} 1 \mathrm{~B}$ & $-\mathrm{C} 6 \mathrm{~B}$ & $-C 5 B$ & $179.09(15)$ \\
\hline $\mathrm{C} 1 \mathrm{~A}$ & $-\mathrm{C} 2 \mathrm{~A}$ & $-C 3 A$ & $-\mathrm{C} 4 \mathrm{~A}$ & $-0.1(3)$ \\
\hline $\mathrm{C} 1 \mathrm{~B}$ & $-\mathrm{C} 2 \mathrm{~B}$ & $-C 3 B$ & $-C 4 B$ & $0.5(3)$ \\
\hline $\mathrm{C} 2 \mathrm{~A}$ & $-\mathrm{C} 3 \mathrm{~A}$ & $-\mathrm{C} 4 \mathrm{~A}$ & $-\mathrm{C} 5 \mathrm{~A}$ & $-0.9(3)$ \\
\hline $\mathrm{C} 2 \mathrm{~B}$ & $-C 3 B$ & $-C 4 B$ & $-C 5 B$ & $-0.7(3)$ \\
\hline C3A & $-\mathrm{C} 4 \mathrm{~A}$ & $-\mathrm{C} 5 \mathrm{~A}$ & $-\mathrm{C} 6 \mathrm{~A}$ & $-0.1(3)$ \\
\hline $\mathrm{C} 3 \mathrm{~B}$ & $-C 4 B$ & $-\mathrm{C} 5 \mathrm{~B}$ & $-\mathrm{C} 6 \mathrm{~B}$ & $-0.4(3)$ \\
\hline $\mathrm{C} 4 \mathrm{~A}$ & $-\mathrm{C} 5 \mathrm{~A}$ & $-\mathrm{C} 6 \mathrm{~A}$ & $-C 7 A$ & $-176.68(15)$ \\
\hline $\mathrm{C} 4 \mathrm{~A}$ & $-\mathrm{C} 5 \mathrm{~A}$ & $-\mathrm{C} 6 \mathrm{~A}$ & $-C 1 A$ & $2.0(2)$ \\
\hline $\mathrm{C} 4 \mathrm{~B}$ & $-\mathrm{C} 5 \mathrm{~B}$ & $-\mathrm{C} 6 \mathrm{~B}$ & $-C 1 B$ & $1.7(2)$ \\
\hline $\mathrm{C} 4 \mathrm{~B}$ & $-C 5 B$ & $-C 6 B$ & $-C 7 B$ & $-176.74(16)$ \\
\hline C5A & $-C 6 A$ & $-C 7 A$ & $-\mathrm{N} 1 \mathrm{~A}$ & $-177.19(16)$ \\
\hline $\mathrm{C} 1 \mathrm{~A}$ & $-\mathrm{C} 6 \mathrm{~A}$ & $-\mathrm{C} 7 \mathrm{~A}$ & $-02 \mathrm{~A}$ & $-174.04(14)$ \\
\hline $\mathrm{C} 1 \mathrm{~A}$ & $-\mathrm{C} 6 \mathrm{~A}$ & $-C 7 A$ & $-N 1 A$ & $4.1(3)$ \\
\hline C5A & $-\mathrm{C} 6 \mathrm{~A}$ & $-C 7 A$ & $-02 \mathrm{~A}$ & $4.7(2)$ \\
\hline $\mathrm{C} 1 \mathrm{~B}$ & $-\mathrm{C} 6 \mathrm{~B}$ & $-C 7 B$ & $-N 1 B$ & $2.7(3)$ \\
\hline C5B & $-\mathrm{C} 6 \mathrm{~B}$ & $-C 7 B$ & $-02 B$ & $3.0(2)$ \\
\hline $\mathrm{C} 1 \mathrm{~B}$ & $-\mathrm{C} 6 \mathrm{~B}$ & $-C 7 B$ & $-02 \mathrm{~B}$ & $-175.47(14)$ \\
\hline C5B & $-\mathrm{C} 6 \mathrm{~B}$ & $-C 7 B$ & $-N 1 B$ & $-178.86(16)$ \\
\hline $\mathrm{O} 2 \mathrm{~A}$ & $-\mathrm{C} 8 \mathrm{~A}$ & $-\mathrm{C} 10 \mathrm{~A}$ & $-\mathrm{N} 1 \mathrm{~A}$ & $23.37(15)$ \\
\hline $\mathrm{O} 2 \mathrm{~A}$ & $-C 8 A$ & $-\mathrm{C} 10 \mathrm{~A}$ & $-\mathrm{C} 9 \mathrm{~A}$ & $142.68(13)$ \\
\hline $\mathrm{O} 2 \mathrm{~A}$ & $-C 8 A$ & $-\mathrm{C} 10 \mathrm{~A}$ & $-\mathrm{C} 11 \mathrm{~A}$ & $-91.06(15)$ \\
\hline $\mathrm{O} 2 \mathrm{~B}$ & $-C 8 B$ & $-\mathrm{C} 10 \mathrm{~B}$ & $-C 9 B$ & $-94.65(16)$ \\
\hline $\mathrm{O} 2 \mathrm{~B}$ & $-C 8 B$ & $-\mathrm{C} 10 \mathrm{~B}$ & $-C 11 B$ & $139.49(14)$ \\
\hline $\mathrm{O} 2 \mathrm{~B}$ & $-C 8 B$ & $-\mathrm{C} 10 \mathrm{~B}$ & $-N 1 B$ & $20.72(15)$ \\
\hline
\end{tabular}



Table s8 - Contact Distances (Angstrom)
for: 4

\begin{tabular}{|c|c|c|c|c|c|}
\hline $\mathrm{V}$ & .H9A & 3.2245 & $\mathrm{O} 2 \mathrm{~B}$ & . $\mathrm{H} 5 \mathrm{BA}$ & 2.4186 \\
\hline $\mathrm{V}$ & .H9C & 3.4727 & $\mathrm{~N} 1 \mathrm{~B}$ & . H11C_b & 2.9266 \\
\hline $\mathrm{V}$ & .H9D & 3.5071 & C3A & . O_e & $3.228(2)$ \\
\hline $\mathrm{V}$ &. $\mathrm{H} 11 \mathrm{~A}$ & 3.5237 & C3A & . C6A_e & $3.540(2)$ \\
\hline $\mathrm{V}$ &. $\mathrm{H} 11 \mathrm{D}$ & 3.1696 & C3A & . C7A_e & $3.386(2)$ \\
\hline $\mathrm{V}$ &. $\mathrm{H} 11 \mathrm{~F}$ & 3.4946 & $\mathrm{C} 4 \mathrm{~A}$ & . O_e & $3.124(2)$ \\
\hline $\mathrm{V}$ & .H3AA_a & 3.3933 & $\mathrm{C} 4 \mathrm{~B}$ & . $C 7 B \_C$ & $3.551(2)$ \\
\hline $\mathrm{V}$ & .H11C_b & 3.3332 & $\mathrm{C} 4 \mathrm{~B}$ &. $\mathrm{O}_{-} \mathrm{C}$ & $3.229(2)$ \\
\hline $\mathrm{O}$ & . $\mathrm{C} 11 \mathrm{~B}$ & $3.338(2)$ & C5B & . $\mathrm{C} 6 \mathrm{~B} \_\mathrm{C}$ & $3.476(2)$ \\
\hline $\mathrm{O}$ & . C3A_a & $3.228(2)$ & $\mathrm{C} 6 \mathrm{~A}$ & . C3A_a & $3.540(2)$ \\
\hline $\mathrm{O}$ & . C4A_a & $3.124(2)$ & $\mathrm{C} 6 \mathrm{~B}$ & . C5B_C & $3.476(2)$ \\
\hline $\mathrm{O}$ & . $C 4 B \_C$ & $3.229(2)$ & C7A & . C3A_a & $3.386(2)$ \\
\hline O1A &. $\mathrm{C} 9 \mathrm{~B}$ & $3.191(2)$ & C7B & . $\mathrm{C} 4 \mathrm{~B} \_\mathrm{C}$ & $3.551(2)$ \\
\hline $\mathrm{O} 1 \mathrm{~A}$ & . C11B & $3.329(2)$ & $\mathrm{C} 8 \mathrm{~B}$ & $.02 A_{f} \mathrm{f}$ & $3.321(2)$ \\
\hline $\mathrm{O} 1 \mathrm{~B}$ & . C11A & $3.160(2)$ & C9A & . C9B_g & $3.433(2)$ \\
\hline $\mathrm{O} 1 \mathrm{~B}$ & . C9A & $3.174(2)$ & $\mathrm{C} 9 \mathrm{~A}$ & $.01 B$ & $3.174(2)$ \\
\hline $\mathrm{O} 2 \mathrm{~A}$ & . $\mathrm{C} 8 \mathrm{~B} \_\mathrm{d}$ & $3.321(2)$ & C9B & $.01 \mathrm{~A}$ & $3.191(2)$ \\
\hline $\mathrm{O}$ & .H4BA_C & 2.4629 & $\mathrm{C} 9 \mathrm{~B}$ & . C9A_h & $3.433(2)$ \\
\hline 0 & .H4AA_a & 2.4836 & $\mathrm{C} 11 \mathrm{~A}$ & $.01 \mathrm{~B}$ & $3.160(2)$ \\
\hline 0 & .H3AA_a & 2.6871 & $\mathrm{C} 11 \mathrm{~B}$ & .0 & $3.338(2)$ \\
\hline $\mathrm{O} 1 \mathrm{~A}$ & .H11D & 2.6361 & $\mathrm{C} 11 \mathrm{~B}$ & $.01 \mathrm{~A}$ & $3.329(2)$ \\
\hline $\mathrm{O} 1 \mathrm{~A}$ & .H9D & 2.4185 & $\mathrm{C} 1 \mathrm{~B}$ & .H8AA_b & 3.0663 \\
\hline O1B & .H9A & 2.4831 & $\mathrm{C} 1 \mathrm{~B}$ &. $\mathrm{H} 11 \mathrm{C} \_\mathrm{b}$ & 2.7563 \\
\hline O1B & . H11A & 2.4169 & $\mathrm{C} 2 \mathrm{~B}$ & .H8AA_b & 3.0723 \\
\hline O1B & . H11C_b & 2.6649 & C3A & .H3BA_b & 2.9643 \\
\hline $\mathrm{O} 2 \mathrm{~A}$ & . H5AA & 2.4086 & $\mathrm{C} 3 \mathrm{~B}$ & .H8AA_b & 3.0146 \\
\hline $\mathrm{O} 2 \mathrm{~A}$ &. $\mathrm{H} 11 \mathrm{~B}$ & 2.9021 & $\mathrm{C} 4 \mathrm{~A}$ &. $\mathrm{H} 3 \mathrm{BA} \_\mathrm{b}$ & 2.8709 \\
\hline
\end{tabular}




\begin{tabular}{|c|c|c|c|c|c|}
\hline $\mathrm{O} 2 \mathrm{~A}$ & . H8BB_d & 2.7933 & $\mathrm{C} 4 \mathrm{~B}$ & .H8AA_b & 2.9573 \\
\hline C5A & .H3BA_b & 2.9178 & H8AB &. $\mathrm{H} 9 \mathrm{C}$ & 2.4232 \\
\hline C5A & .H2AA_a & 3.0313 & H3BA & .C3A_i & 2.9643 \\
\hline $\mathrm{C} 5 \mathrm{~B}$ & .H8AA_b & 2.9295 & Н3BA & . C4A_i & 2.8709 \\
\hline $\mathrm{C} 6 \mathrm{~A}$ & .H3BA_b & 3.0704 & H3BA & . C5A_i & 2.9178 \\
\hline C6A & .H3AA_a & 2.9140 & Н3BA & . C6A_i & 3.0704 \\
\hline $\mathrm{C} 6 \mathrm{~B}$ & . H8AA_b & 2.9677 & $\mathrm{H} 4 \mathrm{BA}$ &. $\mathrm{O}_{-} \mathrm{C}$ & 2.4629 \\
\hline $\mathrm{C} 6 \mathrm{~B}$ & . H11C_b & 2.9118 & H9A &. $\mathrm{V}$ & 3.2245 \\
\hline C7A & . H11B & 2.9588 & H9A & $.01 B$ & 2.4831 \\
\hline C7A & .H3AA_a & 2.9581 & H9A & . H11A & 2.5637 \\
\hline $\mathrm{C} 7 \mathrm{~B}$ & . H9E & 3.0138 & H9B &. $\mathrm{H} 11 \mathrm{C}$ & 2.5741 \\
\hline C7B & . H11C_b & 2.9397 & $\mathrm{H} 9 \mathrm{C}$ &. $\mathrm{V}$ & 3.4727 \\
\hline C9A & .H9D_g & 3.0515 & $\mathrm{H} 9 \mathrm{C}$ & .C9B_g & 3.0477 \\
\hline $\mathrm{C} 9 \mathrm{~B}$ & .H9C_h & 3.0477 & $\mathrm{H} 9 \mathrm{C}$ &. $\mathrm{H} 8 \mathrm{AB}$ & 2.4232 \\
\hline $\mathrm{H} 2 \mathrm{AA}$ & . C5A_e & 3.0313 & H9D &. $\mathrm{V}$ & 3.5071 \\
\hline H3AA & . V_e & 3.3933 & H9D & $.01 \mathrm{~A}$ & 2.4185 \\
\hline H3AA & . O_e & 2.6871 & H9D & . C9A_h & 3.0515 \\
\hline H3AA & . C6A_e & 2.9140 & H9D &. $\mathrm{H} 11 \mathrm{D}$ & 2.5521 \\
\hline H3AA & . C7A_e & 2.9581 & H9E &. $\mathrm{C} 7 \mathrm{~B}$ & 3.0138 \\
\hline $\mathrm{H} 4 \mathrm{AA}$ & . O_e & 2.4836 & H9E & . H8BA & 2.5236 \\
\hline H5AA & $.02 \mathrm{~A}$ & 2.4086 & H9F & . H8BA & 2.5345 \\
\hline $\mathrm{H} 8 \mathrm{AA}$ & . H11B & 2.5589 & H9F &. $\mathrm{H} 11 \mathrm{E}$ & 2.5507 \\
\hline $\mathrm{H} 8 \mathrm{AA}$ &. $\mathrm{H} 11 \mathrm{C}$ & 2.5656 & H5BA & $.02 \mathrm{~B}$ & 2.4186 \\
\hline $\mathrm{H} 8 \mathrm{AA}$ & . $\mathrm{C} \mathrm{B}_{2} \mathrm{i}$ & 3.0663 & H8BA & . H9E & 2.5236 \\
\hline $\mathrm{H} 8 \mathrm{AA}$ & . $\mathrm{C}_{2} \mathrm{~B}_{-} \mathrm{i}$ & 3.0723 & H8BA &. $\mathrm{H} 9 \mathrm{~F}$ & 2.5345 \\
\hline $\mathrm{H} 8 \mathrm{AA}$ & . $\mathrm{C}_{3} \mathrm{~B}_{2} i$ & 3.0146 & $\mathrm{H} 11 \mathrm{~A}$ &. $\mathrm{~V}$ & 3.5237 \\
\hline $\mathrm{H} 8 \mathrm{AA}$ & . $\mathrm{C}_{4} \mathrm{~B}_{-} \mathrm{i}$ & 2.9573 & $\mathrm{H} 11 \mathrm{~A}$ & $.01 B$ & 2.4169 \\
\hline H8AA & . $\mathrm{C}^{5} \mathrm{~B}_{-} \mathrm{i}$ & 2.9295 & $\mathrm{H} 11 \mathrm{~A}$ & .H9A & 2.5637 \\
\hline
\end{tabular}




\begin{tabular}{|c|c|c|c|c|c|}
\hline H8AA & . $\mathrm{C} 6 \mathrm{~B}_{-} \mathrm{i}$ & 2.9677 & $\mathrm{H} 11 \mathrm{~B}$ & $.02 \mathrm{~A}$ & 2.9021 \\
\hline $\mathrm{H} 11 \mathrm{~B}$ & . C7A & 2.9588 & $\mathrm{H} 11 \mathrm{C}$ & . C7B_i & 2.9398 \\
\hline $\mathrm{H} 11 \mathrm{~B}$ & . H8AA & 2.5589 & $\mathrm{H} 11 \mathrm{D}$ &. $\mathrm{V}$ & 3.1696 \\
\hline $\mathrm{H} 11 \mathrm{C}$ & . H8AA & 2.5656 & $\mathrm{H} 11 \mathrm{D}$ & $.01 \mathrm{~A}$ & 2.6361 \\
\hline $\mathrm{H} 11 \mathrm{C}$ & . H9B & 2.5741 & $\mathrm{H} 11 \mathrm{D}$ & .H9D & 2.5521 \\
\hline $\mathrm{H} 11 \mathrm{C}$ & . $V_{-} i$ & 3.3333 & $\mathrm{H} 11 \mathrm{E}$ & .H9F & 2.5507 \\
\hline $\mathrm{H} 11 \mathrm{C}$ & $.01 B \_i$ & 2.6649 & $\mathrm{H} 11 \mathrm{~F}$ &. $\mathrm{~V}$ & 3.4946 \\
\hline $\mathrm{H} 11 \mathrm{C}$ & .N1B_i & 2.9266 & $\mathrm{H} 11 \mathrm{~F}$ & . H8BB & 2.4271 \\
\hline $\mathrm{H} 11 \mathrm{C}$ & . $\mathrm{C} 1 \mathrm{~B}_{-} \mathrm{i}$ & 2.7563 & $\mathrm{H} 8 \mathrm{BB}$ &. $\mathrm{H} 11 \mathrm{~F}$ & 2.4271 \\
\hline $\mathrm{H} 11 \mathrm{C}$ & . $\mathrm{C} 6 \mathrm{~B}$ & 2.9118 & $\mathrm{H} 8 \mathrm{BB}$ & $.02 \mathrm{~A} f$ & 2.7933 \\
\hline
\end{tabular}




\begin{tabular}{|c|c|c|c|c|c|c|c|c|}
\hline $\mathrm{C} 4 \mathrm{~A}$ & -- & $\mathrm{H} 4 \mathrm{AA}$ & .0 & 0.9500 & 2.4800 & $3.124(2)$ & 125.00 & $2 \_645$ \\
\hline $\mathrm{C} 5 \mathrm{~A}$ & -- & H5AA & $\mathrm{O} 2 \mathrm{~A}$ & 0.9500 & 2.4100 & $2.743(2)$ & 100.00 & . \\
\hline C4B & -- & H4 BA & .. 0 & 0.9500 & 2.4600 & $3.229(2)$ & 138.00 & 3_765 \\
\hline C9A & -- & H9A & . O1B & 0.9800 & 2.4800 & $3.174(2)$ & 127.00 & . \\
\hline C9B & -- & H9D & O1A & 0.9800 & 2.4200 & $3.191(2)$ & 135.00 & - \\
\hline C5B & -- & H5BA & $\mathrm{O} 2 \mathrm{~B}$ & 0.9500 & 2.4200 & $2.750(2)$ & 100.00 & - \\
\hline C11A & -- & $\mathrm{H} 11 \mathrm{~A}$ & O1B & 0.9800 & 2.4200 & $3.160(2)$ & 132.00 & . \\
\hline
\end{tabular}


Translation of Symmetry Code to Equiv.Pos
$\mathrm{a}=[2655.00]=1-\mathrm{x}, 1 / 2+\mathrm{y}, 1 / 2-\mathrm{z}$
$\mathrm{b}=[2745.00]=2-\mathrm{x},-1 / 2+\mathrm{y}, 1 / 2-\mathrm{z}$
$\mathrm{c}=[3765.00]=2-\mathrm{x}, 1-\mathrm{y},-\mathrm{z}$
$\mathrm{d}=[4555.00]=\mathrm{x}, 1 / 2-\mathrm{y}, 1 / 2+\mathrm{z}$
$e=[2645.00]=1-x,-1 / 2+y, 1 / 2-z$
$\mathrm{f}=[4554.00]=\mathrm{x}, 1 / 2-\mathrm{y},-1 / 2+\mathrm{z}$
$\mathrm{g}=[1565.00]=\mathrm{x}, 1+\mathrm{y}, \mathrm{z}$
$\mathrm{h}=\left[\begin{array}{ll}1545.00\end{array}\right]=\mathrm{x},-1+\mathrm{y}, \mathrm{z}$
$i=[2755.00]=2-x, 1 / 2+y, 1 / 2-z$ 
Table S1 - Crystal Data and Details of the Structure Determination
for: 5 Crystal Data

Formula

Formula Weight

Crystal system

Space group

$\mathrm{a}, \mathrm{b}, \mathrm{c}$ [Angstrom]

$\mathrm{V}$ [Ang**3]

Z

$\mathrm{D}(\mathrm{calc}) \quad[\mathrm{g} / \mathrm{Cm} * * 3]$

$\mathrm{Mu}(\mathrm{MoKa}) \quad[/ \mathrm{mm}]$

$F(000)$

Crystal size [mm]
C22 H24 Mo N2 O6

508.37

Orthorhombic

C2221

(No. 20)

$10.585(2)$

$14.807(3)$

$27.670(6)$

$4336.8(15)$

8

1.557

0.646

2080

$0.30 \times 0.40 \times 0.60$

Data Collection
Temperature (K)

Radiation [Angstrom]

Theta Min-Max [Deg]

Dataset

Tot., Uniq. Data, R(int)

Observed data [I $>2.0 \operatorname{sigma(I)]}$
293

MoKa

0.71073

$2.4, \quad 27.5$

$0: 13 ; 0: 19 ; 0: 35$

$2681, \quad 2681, \quad 0.000$

1464

Refinement

Nref, Npar

$2681, \quad 281$

R， WR2， S

$0.0617,0.1905,1.03$

$\mathrm{W}=1 /\left[\backslash \mathrm{S}^{\wedge} 2^{\wedge}\left(\mathrm{FO}^{\wedge} 2^{\wedge}\right)+(0.0355 \mathrm{P})^{\wedge} 2^{\wedge}+93.0134 \mathrm{P}\right] \quad \operatorname{WHERE} \quad \mathrm{P}=\left(\mathrm{FO} \mathrm{O}^{\wedge} 2^{\wedge}+\mathrm{FC}^{\wedge} 2^{\wedge}\right) /$

Max. and Av. Shift/Error

$0.08,0.01$

Flack $\mathrm{x}$

$-0.05(16)$

Min. and Max. Resd. Dens. [e/Ang^3]

$-0.80,0.95$ 
Table S2 - Final Coordinates and Equivalent Isotropic Displacement Parameters of the non-Hydrogen atoms

for: 5

\begin{tabular}{|c|c|c|c|c|}
\hline Atom & $\mathrm{x}$ & $y$ & z & $\mathrm{U}(\mathrm{eq}) \quad\left[\mathrm{Ang}^{\wedge} 2\right]$ \\
\hline---- & --- & --- & --- & ---------- \\
\hline Mo1A & $0.60247(15)$ & 1 & $1 / 2$ & $0.0341(5)$ \\
\hline O1A & $0.6991(11)$ & $0.9204(7)$ & $0.4771(4)$ & $0.052(4)$ \\
\hline $\mathrm{O} 2 \mathrm{~A}$ & $0.3219(10)$ & $0.7724(6)$ & $0.4857(4)$ & $0.045(3)$ \\
\hline O3A & $0.5638(10)$ & $0.9445(6)$ & $0.5615(4)$ & $0.045(3)$ \\
\hline $\mathrm{N} 1 \mathrm{~A}$ & $0.4359(10)$ & 0.8985 ( 8 ) & $0.4795(4)$ & $0.034(4)$ \\
\hline $\mathrm{C} 1 \mathrm{~A}$ & $0.5302(13)$ & $0.8646(8)$ & $0.5779(5)$ & $0.032(5)$ \\
\hline $\mathrm{C} 2 \mathrm{~A}$ & $0.5666(14)$ & $0.8404(9)$ & $0.6253(5)$ & $0.036(4)$ \\
\hline C3A & $0.5287(17)$ & 0.7587 (11) & $0.6427(5)$ & $0.049(6)$ \\
\hline $\mathrm{C} 4 \mathrm{~A}$ & $0.4555(18)$ & $0.6982(12)$ & $0.6193(7)$ & $0.064(7)$ \\
\hline C5A & $0.4164(16)$ & $0.7240(10)$ & $0.5730(6)$ & $0.046(5)$ \\
\hline C6A & $0.4514(12)$ & $0.8051(10)$ & $0.5523(5)$ & $0.033(4)$ \\
\hline C7A & $0.4053(12)$ & $0.8300(8)$ & $0.5038(6)$ & $0.034(4)$ \\
\hline C8A & $0.2957(17)$ & 0.8038 (11) & $0.4369(7)$ & $0.060(7)$ \\
\hline C9A & $0.2706(16)$ & 0.9715 (11) & $0.4296(6)$ & $0.055(6)$ \\
\hline C10A & $0.3632(15)$ & $0.8944(9)$ & $0.4317(6)$ & $0.043(5)$ \\
\hline C11A & $0.4512(18)$ & 0.8901 ( 12 ) & $0.3889(7)$ & $0.066(7)$ \\
\hline Mo1B & 1 & $0.81570(13)$ & $3 / 4$ & $0.0425(6)$ \\
\hline O1B & $1.1093(16)$ & 0.7456 ( 8 ) & $0.7708(4)$ & $0.073(5)$ \\
\hline $\mathrm{O} 2 \mathrm{~B}$ & $0.7758(11)$ & $1.0194(7)$ & $0.6634(4)$ & $0.052(4)$ \\
\hline $\mathrm{O} 3 \mathrm{~B}$ & $1.0810(10)$ & $0.8428(8)$ & $0.6895(4)$ & $0.054(4)$ \\
\hline N1B & $0.8727(11)$ & 0.9376 ( 8 ) & $0.7218(4)$ & $0.034(4)$ \\
\hline $\mathrm{C} 1 \mathrm{~B}$ & $1.0387(13)$ & $0.8659(10)$ & $0.6468(7)$ & $0.045(6)$ \\
\hline $\mathrm{C} 2 \mathrm{~B}$ & $1.1037(15)$ & $0.8303(11)$ & $0.6046(5)$ & $0.044(5)$ \\
\hline $\mathrm{C} 3 \mathrm{~B}$ & $1.0658(18)$ & $0.8517(13)$ & $0.5588(7)$ & $0.062(7)$ \\
\hline $\mathrm{C} 4 \mathrm{~B}$ & $0.964(2)$ & $0.9050(13)$ & $0.5516(7)$ & $0.066(8)$ \\
\hline
\end{tabular}


Table S2 - Final Coordinates and Equivalent Isotropic Displacement Parameters of the non-Hydrogen atoms (continued) for: 5

\begin{tabular}{|c|c|c|c|c|}
\hline Atom & $\mathrm{x}$ & Y & z & $\mathrm{U}(\mathrm{eq}) \quad\left[\mathrm{Ang}^{\wedge} 2\right]$ \\
\hline--- & --- & --- & --- & ---------- \\
\hline C5B & $0.8999(19)$ & $0.9401(11)$ & $0.5892(6)$ & $0.052(6)$ \\
\hline $\mathrm{C} 6 \mathrm{~B}$ & $0.9316(15)$ & $0.9182(10)$ & $0.6361(6)$ & $0.044(5)$ \\
\hline C7B & $0.8599(14)$ & $0.9563(10)$ & $0.6772(5)$ & $0.039(5)$ \\
\hline C8B & $0.7231(19)$ & $1.0525(14)$ & $0.7081(6)$ & $0.065(8)$ \\
\hline C9B & $0.6761(17)$ & $0.9339(14)$ & $0.7683(7)$ & $0.070(7)$ \\
\hline $\mathrm{C} 10 \mathrm{~B}$ & $0.7772(12)$ & $0.9939(12)$ & $0.7486(7)$ & $0.050(5)$ \\
\hline C11B & $0.8359(17)$ & $1.0542(13)$ & $0.7867(7)$ & $0.061(7)$ \\
\hline
\end{tabular}


Table S3 - Hydrogen Atom Positions and Isotropic Displacement Parameters

for : 5

\begin{tabular}{|c|c|c|c|c|c|}
\hline Atom & $x$ & Y & z & U (iso) & {$\left[\mathrm{Ang}^{\wedge} 2\right]$} \\
\hline---- & --- & --- & --- & ----- & ----- \\
\hline $\mathrm{H} 2 \mathrm{AA}$ & 0.61520 & 0.87920 & 0.64410 & & 0.0440 \\
\hline H3AA & 0.55570 & 0.74320 & 0.67360 & & 0.0580 \\
\hline $\mathrm{H} 4 \mathrm{AA}$ & 0.43280 & 0.64320 & 0.63290 & & 0.0770 \\
\hline $\mathrm{H} 5 \mathrm{AA}$ & 0.36480 & 0.68510 & 0.55550 & & 0.0540 \\
\hline H8AA & 0.20550 & 0.81100 & 0.43210 & & 0.0720 \\
\hline $\mathrm{H} 8 \mathrm{AB}$ & 0.32730 & 0.76090 & 0.41330 & & 0.0720 \\
\hline H9A & 0.31580 & 1.02770 & 0.42830 & & 0.0820 \\
\hline H9B & 0.21800 & 0.97040 & 0.45780 & & 0.0820 \\
\hline $\mathrm{H} 9 \mathrm{C}$ & 0.21890 & 0.96560 & 0.40120 & & 0.0820 \\
\hline $\mathrm{H} 11 \mathrm{~A}$ & 0.49540 & 0.94650 & 0.38590 & & 0.0990 \\
\hline $\mathrm{H} 11 \mathrm{~B}$ & 0.40340 & 0.87900 & 0.36010 & & 0.0990 \\
\hline $\mathrm{H} 11 \mathrm{C}$ & 0.51110 & 0.84220 & 0.39360 & & 0.0990 \\
\hline $\mathrm{H} 2 \mathrm{BA}$ & 1.17250 & 0.79210 & 0.60880 & & 0.0530 \\
\hline H3 BA & 1.11040 & 0.82950 & 0.53240 & & 0.0750 \\
\hline $\mathrm{H} 4 \mathrm{BA}$ & 0.93780 & 0.91760 & 0.52030 & & 0.0790 \\
\hline H9D & 0.64070 & 0.89860 & 0.74250 & & 0.1050 \\
\hline H9E & 0.61110 & 0.97010 & 0.78280 & & 0.1050 \\
\hline H9F & 0.71160 & 0.89440 & 0.79220 & & 0.1050 \\
\hline H5BA & 0.83340 & 0.97970 & 0.58350 & & 0.0630 \\
\hline H8BA & 0.63170 & 1.04800 & 0.70740 & & 0.0790 \\
\hline $\mathrm{H} 11 \mathrm{D}$ & 0.87010 & 1.01770 & 0.81210 & & 0.0920 \\
\hline $\mathrm{H} 11 \mathrm{E}$ & 0.77250 & 1.09380 & 0.79960 & & 0.0920 \\
\hline $\mathrm{H} 11 \mathrm{~F}$ & 0.90220 & 1.08940 & 0.77230 & & 0.0920 \\
\hline H8BB & 0.74610 & 1.11530 & 0.71290 & & 0.0790 \\
\hline
\end{tabular}

The Temperature Factor has the Form of $\operatorname{Exp}(-\mathrm{T})$ Where $\mathrm{T}=8 *(\mathrm{Pi} * 2) * \mathrm{U} *(\mathrm{Sin}($ Theta $) /$ Lambda $) * * 2$ for Isotropic Atoms 


\begin{tabular}{|c|c|c|c|c|c|c|}
\hline Atom & $\mathrm{U}(1,1)$ or $\mathrm{U}$ & $\mathrm{J} \quad \mathrm{U}(2,2)$ & $\mathrm{U}(3,3)$ & $\mathrm{U}(2,3)$ & $\mathrm{U}(1,3)$ & $\mathrm{U}(1,2)$ \\
\hline--- & ---- & ----- & ---- & ----- & ----- & ----- \\
\hline Mo1A & $0.0271(8)$ & $0.0324(8)$ & $0.0429(10)$ & $0.0093(9)$ & 0 & 0 \\
\hline $\mathrm{O} 1 \mathrm{~A}$ & $0.050(7)$ & $0.049(7)$ & $0.058(7)$ & $0.009(6)$ & $-0.002(6)$ & $0.016(6)$ \\
\hline $\mathrm{O} 2 \mathrm{~A}$ & $0.045(6)$ & $0.040(5)$ & $0.050(7)$ & $-0.006(5)$ & $-0.001(5)$ & $-0.012(5)$ \\
\hline O3A & $0.055(7)$ & $0.034(5)$ & $0.045(6)$ & $0.008(5)$ & $-0.032(5)$ & $-0.017(5)$ \\
\hline $\mathrm{N} 1 \mathrm{~A}$ & $0.024(6)$ & $0.037(7)$ & $0.041(7)$ & $0.001(5)$ & $-0.003(5)$ & $0.002(5)$ \\
\hline $\mathrm{C} 1 \mathrm{~A}$ & $0.049(10)$ & $0.028(7)$ & $0.020(6)$ & $-0.005(5)$ & $-0.027(6)$ & $0.001(6)$ \\
\hline $\mathrm{C} 2 \mathrm{~A}$ & $0.052(9)$ & $0.045(8)$ & $0.012(6)$ & $-0.005(6)$ & $0.001(6)$ & $-0.001(7)$ \\
\hline C3A & $0.076(13)$ & $0.068(11)$ & $0.002(5)$ & $0.004(6)$ & $0.007(7)$ & $0.001(10)$ \\
\hline $\mathrm{C} 4 \mathrm{~A}$ & $0.079(14)$ & $0.049(10)$ & $0.063(12)$ & $0.002(10)$ & $0.033(10)$ & $-0.010(10)$ \\
\hline C5A & $0.046(10)$ & $0.040(8)$ & $0.051(10)$ & $0.010(8)$ & $-0.001(8)$ & $-0.003(8)$ \\
\hline $\mathrm{C} 6 \mathrm{~A}$ & $0.033(7)$ & $0.040(8)$ & $0.026(7)$ & $0.004(7)$ & $0.011(6)$ & $0.001(6)$ \\
\hline C7A & $0.027(6)$ & $0.025(6)$ & $0.049(8)$ & $-0.009(7)$ & $0.009(8)$ & $0.000(6)$ \\
\hline $\mathrm{C} 8 \mathrm{~A}$ & $0.060(11)$ & $0.047(10)$ & $0.074(13)$ & $0.002(10)$ & $-0.035(10)$ & $0.000(9)$ \\
\hline C9A & $0.054(10)$ & $0.058(11)$ & $0.053(11)$ & $0.003(8)$ & $-0.013(9)$ & $0.017(8)$ \\
\hline C10A & $0.048(10)$ & $0.032(8)$ & $0.050(10)$ & $-0.008(7)$ & $-0.015(8)$ & $0.007(7)$ \\
\hline C11A & $0.070(13)$ & $0.060(11)$ & $0.067(13)$ & $-0.001(10)$ & $-0.008(11)$ & $0.005(10)$ \\
\hline Mo1B & $0.0491(11)$ & $0.0450(11)$ & $0.0335(10)$ & 0 & $0.0025(10)$ & 0 \\
\hline O1B & $0.109(11)$ & $0.062(7)$ & $0.048(7)$ & $0.008(6)$ & $0.003(9)$ & $0.020(9)$ \\
\hline O2B & $0.054(7)$ & $0.056(8)$ & $0.046(7)$ & $0.003(6)$ & $-0.011(6)$ & $0.012(6)$ \\
\hline O3B & $0.034(6)$ & $0.070(8)$ & $0.059(7)$ & $0.002(6)$ & $0.002(6)$ & $0.019(6)$ \\
\hline N1B & $0.025(6)$ & $0.057(8)$ & $0.019(6)$ & $-0.005(6)$ & $-0.006(5)$ & $0.003(6)$ \\
\hline $\mathrm{C} 1 \mathrm{~B}$ & $0.026(8)$ & $0.033(8)$ & $0.077(13)$ & $-0.001(9)$ & $-0.005(8)$ & $-0.008(6)$ \\
\hline $\mathrm{C} 2 \mathrm{~B}$ & $0.031(7)$ & $0.071(10)$ & $0.030(7)$ & $-0.009(8)$ & $0.005(7)$ & $0.002(9)$ \\
\hline C3B & $0.054(12)$ & $0.085(14)$ & $0.048(11)$ & $-0.021(10)$ & $0.013(9)$ & $-0.030(11)$ \\
\hline C4B & $0.076(15)$ & $0.080(14)$ & $0.041(10)$ & $0.013(10)$ & $-0.011(10)$ & $-0.026(12)$ \\
\hline $\mathrm{C} 5$ & $0.054(10)$ & $0.050(9)$ & $0.053(11)$ & $0.006(8)$ & $-0.002(10)$ & $-0.012(9)$ \\
\hline
\end{tabular}




$\begin{array}{lrrrrrr}\text { C6B } & 0.042(9) & 0.039(8) & 0.051(10) & 0.005(7) & 0.003(8) & -0.006(7) \\ \text { C7B } & 0.040(8) & 0.047(8) & 0.029(8) & -0.004(7) & -0.015(7) & -0.006(7) \\ \text { C8B } & 0.065(13) & 0.092(15) & 0.039(11) & 0.001(11) & -0.010(10) & 0.017(12) \\ \text { C9B } & 0.043(10) & 0.112(16) & 0.056(12) & 0.003(11) & 0.017(9) & 0.011(11) \\ \text { C10B } & 0.026(7) & 0.074(10) & 0.051(8) & -0.017(15) & 0.011(8) & 0.000(9) \\ \text { C11B } & 0.048(10) & 0.082(13) & 0.054(12) & -0.015(11) & 0.007(9) & 0.002(10)\end{array}$



The Temperature Factor has the Form of $\operatorname{Exp}(-\mathrm{T})$ Where

$\mathrm{T}=8 *(\mathrm{Pi} * 2) * \mathrm{U} *(\operatorname{Sin}($ Theta $) /$ Lambda $) * * 2$ for Isotropic Atoms

$T=2 *(P i * 2) * \operatorname{Sumij}(h(i) * h(j) * U(i, j) * A s t a r(i) * A s t a r(j))$, for Anisotropic Atoms. Astar(i) are Reciprocal Axial Lengths and $h(i)$ are the Reflection Indices. 

Table S5 - Bond Distances (Angstrom)
for: 5

\begin{tabular}{|c|c|c|c|c|c|}
\hline Mo1A & $-01 A$ & $1.684(11)$ & $\mathrm{C} 4 \mathrm{~A}$ & $-\mathrm{H} 4 \mathrm{AA}$ & 0.9287 \\
\hline Mo1A & $-03 A$ & $1.934(11)$ & C5A & $-\mathrm{H} 5 \mathrm{AA}$ & 0.9298 \\
\hline Mo1A & $-\mathrm{N} 1 \mathrm{~A}$ & $2.385(11)$ & C8A & $-\mathrm{H} 8 \mathrm{AB}$ & 0.9705 \\
\hline Mo1B & $-N 1 B$ & $2.384(12)$ & C8A & $-H 8 A A$ & 0.9698 \\
\hline Mo1B & $-01 B$ & $1.657(15)$ & C9A & $-\mathrm{H} 9 \mathrm{~B}$ & 0.9587 \\
\hline Mo1B & $-03 B$ & $1.923(11)$ & C9A & $-\mathrm{H} 9 \mathrm{C}$ & 0.9616 \\
\hline $\mathrm{O} 2 \mathrm{~A}$ & $-C 8 A$ & $1.45(2)$ & C9A & $-\mathrm{H} 9 \mathrm{~A}$ & 0.9606 \\
\hline $\mathrm{O} 2 \mathrm{~A}$ & $-C 7 A$ & $1.326(16)$ & $\mathrm{C} 11 \mathrm{~A}$ & $-\mathrm{H} 11 \mathrm{~B}$ & 0.9582 \\
\hline O3A & $-C 1 A$ & $1.316(15)$ & $\mathrm{C} 11 \mathrm{~A}$ & $-\mathrm{H} 11 \mathrm{C}$ & 0.9602 \\
\hline $\mathrm{O} 2 \mathrm{~B}$ & $-C 8 B$ & $1.44(2)$ & $\mathrm{C} 11 \mathrm{~A}$ & $-\mathrm{H} 11 \mathrm{~A}$ & 0.9608 \\
\hline $\mathrm{O} 2 \mathrm{~B}$ & $-C 7 B$ & $1.346(18)$ & $\mathrm{C} 1 \mathrm{~B}$ & $-\mathrm{C} 2 \mathrm{~B}$ & $1.45(2)$ \\
\hline O3B & $-C 1 B$ & $1.31(2)$ & $\mathrm{C} 1 \mathrm{~B}$ & $-C 6 B$ & $1.40(2)$ \\
\hline $\mathrm{N} 1 \mathrm{~A}$ & $-\mathrm{C} 10 \mathrm{~A}$ & $1.53(2)$ & $\mathrm{C} 2 \mathrm{~B}$ & $-C 3 B$ & $1.37(2)$ \\
\hline $\mathrm{N} 1 \mathrm{~A}$ & $-C 7 A$ & $1.259(18)$ & $\mathrm{C} 3 \mathrm{~B}$ & $-C 4 B$ & $1.35(3)$ \\
\hline $\mathrm{N} 1 \mathrm{~B}$ & $-C 7 B$ & $1.272(18)$ & $\mathrm{C} 4 \mathrm{~B}$ & $-C 5 B$ & $1.35(3)$ \\
\hline $\mathrm{N} 1 \mathrm{~B}$ & $-\mathrm{C} 10 \mathrm{~B}$ & $1.51(2)$ & $\mathrm{C} 5 \mathrm{~B}$ & $-C 6 B$ & $1.38(2)$ \\
\hline $\mathrm{C} 1 \mathrm{~A}$ & $-\mathrm{C} 6 \mathrm{~A}$ & $1.405(19)$ & $\mathrm{C} 6 \mathrm{~B}$ & $-C 7 B$ & $1.48(2)$ \\
\hline $\mathrm{C} 1 \mathrm{~A}$ & $-\mathrm{C} 2 \mathrm{~A}$ & $1.41(2)$ & $\mathrm{C} 8 \mathrm{~B}$ & $-\mathrm{C} 10 \mathrm{~B}$ & $1.53(3)$ \\
\hline $\mathrm{C} 2 \mathrm{~A}$ & $-C 3 A$ & $1.36(2)$ & $\mathrm{C} 9 \mathrm{~B}$ & $-\mathrm{C} 10 \mathrm{~B}$ & $1.49(2)$ \\
\hline C3A & $-C 4 A$ & $1.35(2)$ & $\mathrm{C} 10 \mathrm{~B}$ & $-\mathrm{C} 11 \mathrm{~B}$ & $1.51(3)$ \\
\hline $\mathrm{C} 4 \mathrm{~A}$ & $-\mathrm{C} 5 \mathrm{~A}$ & $1.40(3)$ & $\mathrm{C} 2 \mathrm{~B}$ & -H2BA & 0.9294 \\
\hline C5A & $-\mathrm{C} 6 \mathrm{~A}$ & $1.38(2)$ & $\mathrm{C} 3 \mathrm{~B}$ & - Н3BA & 0.9298 \\
\hline $\mathrm{C} 6 \mathrm{~A}$ & $-C 7 A$ & $1.47(2)$ & $\mathrm{C} 4 \mathrm{~B}$ & $-\mathrm{H} 4 \mathrm{BA}$ & 0.9283 \\
\hline C8A & $-\mathrm{C} 10 \mathrm{~A}$ & $1.53(2)$ & $\mathrm{C} 5 \mathrm{~B}$ & $-\mathrm{H} 5 \mathrm{BA}$ & 0.9296 \\
\hline $\mathrm{C} 9 \mathrm{~A}$ & $-\mathrm{C} 10 \mathrm{~A}$ & $1.51(2)$ & $\mathrm{C} 8 \mathrm{~B}$ & -H8BA & 0.9700 \\
\hline $\mathrm{C} 10 \mathrm{~A}$ & $-C 11 A$ & $1.51(3)$ & $\mathrm{C} 8 \mathrm{~B}$ & $-\mathrm{H} 8 \mathrm{BB}$ & 0.9704 \\
\hline $\mathrm{C} 2 \mathrm{~A}$ & $-\mathrm{H} 2 \mathrm{AA}$ & 0.9302 & $\mathrm{C} 9 \mathrm{~B}$ & $-\mathrm{H} 9 \mathrm{D}$ & 0.9609 \\
\hline
\end{tabular}




$\begin{array}{llllll}\text { C3A } & -\mathrm{H} 3 \mathrm{AA} & 0.9303 & \mathrm{C} 9 \mathrm{~B} & -\mathrm{H} 9 \mathrm{E} & 0.9600 \\ \mathrm{C} 9 \mathrm{~B} & -\mathrm{H} 9 \mathrm{~F} & 0.9595 & \mathrm{C} 11 \mathrm{~B} & -\mathrm{H} 11 \mathrm{E} & 0.9600 \\ \mathrm{C} 11 \mathrm{~B} & -\mathrm{H} 11 \mathrm{D} & 0.9576 & \mathrm{C} 11 \mathrm{~B} & -\mathrm{H} 11 \mathrm{~F} & 0.9607\end{array}$



Table S6 - Bond Angles (Degrees)
for: 5

\begin{tabular}{|c|c|c|c|c|c|c|c|}
\hline $\mathrm{O} 1 \mathrm{~A}$ & -Mo1A & $-03 A$ & $99.3(5)$ & O3B_b & -Mo1B & $-N 1 B$ & $82.8(4)$ \\
\hline O1A & -Mo1A & $-\mathrm{N} 1 \mathrm{~A}$ & $85.3(5)$ & $\mathrm{N} 1 \mathrm{~B}$ & -Mo1B & $-\mathrm{N} 1 \mathrm{~B} \_\mathrm{b}$ & $81.6(4)$ \\
\hline $\mathrm{O} 1 \mathrm{~A}$ & -Mo1A & $-01 A \_a$ & $105.2(5)$ & C7A & $-02 \mathrm{~A}$ & $-\mathrm{C} 8 \mathrm{~A}$ & $105.8(11)$ \\
\hline $\mathrm{O} 1 \mathrm{~A}$ & $-M o 1 A$ & -03A_a & $95.4(5)$ & $\operatorname{Mo1A}$ & $-03 \mathrm{~A}$ & $-\mathrm{C} 1 \mathrm{~A}$ & $138.0(9)$ \\
\hline O1A & -Mo1A & $-\mathrm{N} 1 \mathrm{~A} \_\mathrm{a}$ & $168.3(5)$ & C7B & $-02 B$ & $-C 8 B$ & $104.4(12)$ \\
\hline O3A & $-\operatorname{Mo1A}$ & $-\mathrm{N} 1 \mathrm{~A}$ & $77.6(4)$ & Mo1B & $-03 B$ & $-\mathrm{C} 1 \mathrm{~B}$ & $133.4(10)$ \\
\hline O1A_a & $-\operatorname{Mo1A}$ & $-03 A$ & $95.4(5)$ & C7A & $-\mathrm{N} 1 \mathrm{~A}$ & $-\mathrm{C} 10 \mathrm{~A}$ & $107.5(11)$ \\
\hline O3A & -Mo1A & -03A_a & $155.6(5)$ & Mo1A & $-\mathrm{N} 1 \mathrm{~A}$ & $-\mathrm{C} 10 \mathrm{~A}$ & $127.0(8)$ \\
\hline O3A & -Mo1A & $-\mathrm{N} 1 \mathrm{~A} \_\mathrm{a}$ & $84.4(4)$ & Mo1A & $-\mathrm{N} 1 \mathrm{~A}$ & $-C 7 A$ & $124.8(9)$ \\
\hline O1A_a & -Mo1A & $-\mathrm{N} 1 \mathrm{~A}$ & $168.3(5)$ & Mo1B & $-\mathrm{N} 1 \mathrm{~B}$ & $-C 7 B$ & $122.9(10)$ \\
\hline O3A_a & -Mo1A & $-\mathrm{N} 1 \mathrm{~A}$ & $84.4(4)$ & C7B & $-N 1 B$ & - C10B & $106.6(12)$ \\
\hline $\mathrm{N} 1 \mathrm{~A}$ & -Mo1A & $-N 1 A_{-} a$ & $84.7(4)$ & Mo1B & $-\mathrm{N} 1 \mathrm{~B}$ & $-\mathrm{C} 10 \mathrm{~B}$ & $129.6(10)$ \\
\hline O1A_a & -Mo1A & $-03 A \_a$ & $99.3(5)$ & O3A & $-C 1 A$ & $-\mathrm{C} 6 \mathrm{~A}$ & $123.4(13)$ \\
\hline O1A_a & -Mo1A & $-\mathrm{N} 1 \mathrm{~A} \_\mathrm{a}$ & $85.3(5)$ & $\mathrm{C} 2 \mathrm{~A}$ & $-C 1 A$ & $-\mathrm{C} 6 \mathrm{~A}$ & $118.1(12)$ \\
\hline O3A_a & -Mo1A & $-\mathrm{N} 1 \mathrm{~A} \_\mathrm{a}$ & $77.6(4)$ & O3A & $-C 1 A$ & $-\mathrm{C} 2 \mathrm{~A}$ & $118.3(12)$ \\
\hline O1B_b & -Mo1B & $-\mathrm{N} 1 \mathrm{~B} \_\mathrm{b}$ & $169.2(6)$ & $\mathrm{C} 1 \mathrm{~A}$ & $-C 2 A$ & $-C 3 A$ & $118.2(13)$ \\
\hline O3B_b & -Mo1B & $-\mathrm{N} 1 \mathrm{~B} \_\mathrm{b}$ & $79.0(4)$ & $\mathrm{C} 2 \mathrm{~A}$ & $-C 3 A$ & $-C 4 A$ & $126.1(15)$ \\
\hline O3B & -Mo1B & $-N 1 B$ & $79.0(4)$ & C3A & $-C 4 A$ & $-\mathrm{C} 5 \mathrm{~A}$ & $115.3(16)$ \\
\hline O1B_b & -Mo1B & $-03 B$ & $98.0(5)$ & $\mathrm{C} 4 \mathrm{~A}$ & $-\mathrm{C} 5 \mathrm{~A}$ & $-\mathrm{C} 6 \mathrm{~A}$ & $122.5(15)$ \\
\hline O1B & -Mo1B & $-03 \mathrm{~B}$ & $97.0(6)$ & $\mathrm{C} 1 \mathrm{~A}$ & $-\mathrm{C} 6 \mathrm{~A}$ & $-C 7 A$ & $119.9(12)$ \\
\hline O1B & -Mo1B & $-N 1 B$ & $169.2(6)$ & C5A & $-\mathrm{C} 6 \mathrm{~A}$ & $-C 7 A$ & $120.4(13)$ \\
\hline O1B & -Mo1B & $-01 B \_b$ & $102.5(7)$ & $\mathrm{C} 1 \mathrm{~A}$ & $-\mathrm{C} 6 \mathrm{~A}$ & $-\mathrm{C} 5 \mathrm{~A}$ & $119.7(13)$ \\
\hline O1B & -Mo1B & $-03 B \_b$ & $98.0(5)$ & N1A & $-C 7 A$ & $-\mathrm{C} 6 \mathrm{~A}$ & $127.0(12)$ \\
\hline O1B & -Mo1B & $-\mathrm{N} 1 \mathrm{~B} \_\mathrm{b}$ & $88.0(5)$ & $\mathrm{O} 2 \mathrm{~A}$ & $-C 7 A$ & $-\mathrm{N} 1 \mathrm{~A}$ & $119.2(14)$ \\
\hline O1B_b & -Mo1B & $-03 B \_b$ & $97.0(6)$ & $\mathrm{O} 2 \mathrm{~A}$ & $-C 7 A$ & $-\mathrm{C} 6 \mathrm{~A}$ & $113.8(12)$ \\
\hline O1B_b & -Mo1B & $-\mathrm{N} 1 \mathrm{~B}$ & $88.0(5)$ & $\mathrm{O} 2 \mathrm{~A}$ & $-\mathrm{C} 8 \mathrm{~A}$ & $-\mathrm{C} 10 \mathrm{~A}$ & $106.2(13)$ \\
\hline O3B & -Mo1B & $-03 B \_b$ & $155.9(5)$ & C8A & - C10A & $-\mathrm{C} 11 \mathrm{~A}$ & $109.0(13)$ \\
\hline
\end{tabular}




\begin{tabular}{|c|c|c|c|c|c|c|c|}
\hline $\mathrm{O} 3 \mathrm{~B}$ & -Mo1B & $-\mathrm{N} 1 \mathrm{~B} \_\mathrm{b}$ & $82.8(4)$ & C9A & $-\mathrm{C} 10 \mathrm{~A}$ & $-\mathrm{C} 11 \mathrm{~A}$ & $113.8(14)$ \\
\hline $\mathrm{N} 1 \mathrm{~A}$ & $-\mathrm{C} 10 \mathrm{~A}$ & $-C 9 A$ & $109.3(12)$ & $\mathrm{H} 11 \mathrm{~A}$ & $-C 11 A$ & $-\mathrm{H} 11 \mathrm{~B}$ & 109.53 \\
\hline $\mathrm{N} 1 \mathrm{~A}$ & $-\mathrm{C} 10 \mathrm{~A}$ & $-C 11 \mathrm{~A}$ & $111.7(13)$ & $\mathrm{O} 3 \mathrm{~B}$ & $-C 1 B$ & $-\mathrm{C} 2 \mathrm{~B}$ & $117.9(13)$ \\
\hline $\mathrm{N} 1 \mathrm{~A}$ & $-\mathrm{C} 10 \mathrm{~A}$ & $-C 8 A$ & $100.9(12)$ & $\mathrm{O} 3 \mathrm{~B}$ & $-\mathrm{C} 1 \mathrm{~B}$ & $-\mathrm{C} 6 \mathrm{~B}$ & $127.6(16)$ \\
\hline $\mathrm{C} 8 \mathrm{~A}$ & $-\mathrm{C} 10 \mathrm{~A}$ & $-C 9 A$ & $111.4(13)$ & $\mathrm{C} 2 \mathrm{~B}$ & $-\mathrm{C} 1 \mathrm{~B}$ & $-\mathrm{C} 6 \mathrm{~B}$ & $114.4(16)$ \\
\hline $\mathrm{C} 1 \mathrm{~A}$ & $-\mathrm{C} 2 \mathrm{~A}$ & $-\mathrm{H} 2 \mathrm{AA}$ & 120.88 & $\mathrm{C} 1 \mathrm{~B}$ & $-\mathrm{C} 2 \mathrm{~B}$ & $-C 3 B$ & $121.4(15)$ \\
\hline C3A & $-\mathrm{C} 2 \mathrm{~A}$ & $-\mathrm{H} 2 \mathrm{AA}$ & 120.90 & $\mathrm{C} 2 \mathrm{~B}$ & $-C 3 B$ & $-C 4 B$ & $120.4(18)$ \\
\hline $\mathrm{C} 2 \mathrm{~A}$ & $-C 3 A$ & $-\mathrm{H} 3 \mathrm{AA}$ & 116.96 & $\mathrm{C} 3 \mathrm{~B}$ & $-C 4 B$ & $-\mathrm{C} 5 \mathrm{~B}$ & $120.9(18)$ \\
\hline $\mathrm{C} 4 \mathrm{~A}$ & $-C 3 A$ & $-\mathrm{H} 3 \mathrm{AA}$ & 116.97 & $\mathrm{C} 4 \mathrm{~B}$ & $-\mathrm{C} 5 \mathrm{~B}$ & $-\mathrm{C} 6 \mathrm{~B}$ & $120.9(17)$ \\
\hline C3A & $-\mathrm{C} 4 \mathrm{~A}$ & $-\mathrm{H} 4 \mathrm{AA}$ & 122.42 & $\mathrm{C} 1 \mathrm{~B}$ & $-\mathrm{C} 6 \mathrm{~B}$ & $-C 5 B$ & $121.6(16)$ \\
\hline $\mathrm{C} 5 \mathrm{~A}$ & $-\mathrm{C} 4 \mathrm{~A}$ & $-\mathrm{H} 4 \mathrm{AA}$ & 122.26 & $\mathrm{C} 1 \mathrm{~B}$ & $-\mathrm{C} 6 \mathrm{~B}$ & $-C 7 B$ & $117.5(15)$ \\
\hline $\mathrm{C} 6 \mathrm{~A}$ & $-\mathrm{C} 5 \mathrm{~A}$ & $-\mathrm{H} 5 \mathrm{AA}$ & 118.70 & C5B & $-\mathrm{C} 6 \mathrm{~B}$ & $-C 7 B$ & $120.6(14)$ \\
\hline $\mathrm{C} 4 \mathrm{~A}$ & $-\mathrm{C} 5 \mathrm{~A}$ & $-\mathrm{H} 5 \mathrm{AA}$ & 118.77 & $\mathrm{O} 2 \mathrm{~B}$ & $-C 7 B$ & $-N 1 B$ & $119.8(13)$ \\
\hline $\mathrm{O} 2 \mathrm{~A}$ & $-C 8 A$ & $-\mathrm{H} 8 \mathrm{AA}$ & 110.48 & $\mathrm{O} 2 \mathrm{~B}$ & $-C 7 B$ & $-\mathrm{C} 6 \mathrm{~B}$ & $112.7(12)$ \\
\hline H8AA & $-\mathrm{C} 8 \mathrm{~A}$ & $-\mathrm{H} 8 \mathrm{AB}$ & 108.61 & $\mathrm{~N} 1 \mathrm{~B}$ & $-C 7 B$ & $-\mathrm{C} 6 \mathrm{~B}$ & $127.5(14)$ \\
\hline $\mathrm{O} 2 \mathrm{~A}$ & $-C 8 A$ & $-\mathrm{H} 8 \mathrm{AB}$ & 110.47 & $\mathrm{O} 2 \mathrm{~B}$ & $-C 8 B$ & $-\mathrm{C} 10 \mathrm{~B}$ & $106.9(15)$ \\
\hline $\mathrm{C} 10 \mathrm{~A}$ & $-C 8 A$ & $-\mathrm{H} 8 \mathrm{AA}$ & 110.56 & $\mathrm{~N} 1 \mathrm{~B}$ & $-\mathrm{C} 10 \mathrm{~B}$ & $-C 8 B$ & $101.8(14)$ \\
\hline $\mathrm{C} 10 \mathrm{~A}$ & $-C 8 A$ & $-\mathrm{H} 8 \mathrm{AB}$ & 110.51 & $\mathrm{~N} 1 \mathrm{~B}$ & $-\mathrm{C} 10 \mathrm{~B}$ & $-C 9 B$ & $109.4(14)$ \\
\hline $\mathrm{C} 10 \mathrm{~A}$ & $-C 9 A$ & $-\mathrm{H} 9 \mathrm{C}$ & 109.46 & $\mathrm{~N} 1 \mathrm{~B}$ & $-\mathrm{C} 10 \mathrm{~B}$ & $-C 11 B$ & $113.2(12)$ \\
\hline $\mathrm{H} 9 \mathrm{~A}$ & $-\mathrm{C} 9 \mathrm{~A}$ & $-\mathrm{H} 9 \mathrm{~B}$ & 109.54 & $\mathrm{C} 8 \mathrm{~B}$ & $-\mathrm{C} 10 \mathrm{~B}$ & $-C 9 B$ & $109.7(13)$ \\
\hline H9A & $-C 9 A$ & $-\mathrm{H} 9 \mathrm{C}$ & 109.36 & $\mathrm{C} 8 \mathrm{~B}$ & $-\mathrm{C} 10 \mathrm{~B}$ & $-\mathrm{C} 11 \mathrm{~B}$ & $109.2(15)$ \\
\hline $\mathrm{C} 10 \mathrm{~A}$ & $-C 9 A$ & $-\mathrm{H} 9 \mathrm{~B}$ & 109.50 & C9B & $-C 10 B$ & $-C 11 B$ & $113.0(16)$ \\
\hline $\mathrm{C} 10 \mathrm{~A}$ & $-C 9 A$ & $-\mathrm{H} 9 \mathrm{~A}$ & 109.51 & $\mathrm{C} 1 \mathrm{~B}$ & $-\mathrm{C} 2 \mathrm{~B}$ & $-\mathrm{H} 2 \mathrm{BA}$ & 119.40 \\
\hline $\mathrm{H} 9 \mathrm{~B}$ & $-C 9 A$ & $-\mathrm{H} 9 \mathrm{C}$ & 109.46 & $\mathrm{C} 3 \mathrm{~B}$ & $-\mathrm{C} 2 \mathrm{~B}$ & $-\mathrm{H} 2 \mathrm{BA}$ & 119.15 \\
\hline $\mathrm{H} 11 \mathrm{~A}$ & $-\mathrm{C} 11 \mathrm{~A}$ & $-\mathrm{H} 11 \mathrm{C}$ & 109.40 & $\mathrm{C} 2 \mathrm{~B}$ & $-C 3 B$ & $-\mathrm{H} 3 \mathrm{BA}$ & 119.84 \\
\hline $\mathrm{H} 11 \mathrm{~B}$ & $-C 11 \mathrm{~A}$ & $-\mathrm{H} 11 \mathrm{C}$ & 109.55 & $\mathrm{C} 4 \mathrm{~B}$ & $-C 3 B$ & $-\mathrm{H} 3 \mathrm{BA}$ & 119.73 \\
\hline $\mathrm{C} 10 \mathrm{~A}$ & $-C 11 A$ & $-\mathrm{H} 11 \mathrm{~A}$ & 109.38 & $\mathrm{C} 3 \mathrm{~B}$ & $-C 4 B$ & $-\mathrm{H} 4 \mathrm{BA}$ & 119.57 \\
\hline C10A & $-C 11 A$ & $-\mathrm{H} 11 \mathrm{~B}$ & 109.52 & $\mathrm{C} 5 \mathrm{~B}$ & $-C 4 B$ & $-\mathrm{H} 4 \mathrm{BA}$ & 119.52 \\
\hline
\end{tabular}




\begin{tabular}{|c|c|c|c|c|c|c|c|}
\hline $\mathrm{C} 10 \mathrm{~A}$ & $-\mathrm{C} 11 \mathrm{~A}$ & $-\mathrm{H} 11 \mathrm{C}$ & 109.43 & $\mathrm{C} 4 \mathrm{~B}$ & $-C 5 B$ & $-\mathrm{H} 5 \mathrm{BA}$ & 119.60 \\
\hline C6B & $-C 5 B$ & $-\mathrm{H} 5 \mathrm{BA}$ & 119.49 & H9D & $-C 9 B$ & $-\mathrm{H} 9 \mathrm{E}$ & 109.56 \\
\hline $\mathrm{O} 2 \mathrm{~B}$ & $-C 8 B$ & $-\mathrm{H} 8 \mathrm{BA}$ & 110.20 & H9D & $-C 9 B$ & $-\mathrm{H} 9 \mathrm{~F}$ & 109.46 \\
\hline $\mathrm{O} 2 \mathrm{~B}$ & $-C 8 B$ & $-\mathrm{H} 8 \mathrm{BB}$ & 110.24 & $\mathrm{H} 9 \mathrm{E}$ & $-C 9 B$ & $-\mathrm{H} 9 \mathrm{~F}$ & 109.45 \\
\hline $\mathrm{C} 10 \mathrm{~B}$ & $-C 8 B$ & $-\mathrm{H} 8 \mathrm{BA}$ & 110.44 & $\mathrm{C} 10 \mathrm{~B}$ & $-C 11 B$ & $-\mathrm{H} 11 \mathrm{D}$ & 109.46 \\
\hline $\mathrm{C} 10 \mathrm{~B}$ & $-C 8 B$ & $-\mathrm{H} 8 \mathrm{BB}$ & 110.46 & $\mathrm{C} 10 \mathrm{~B}$ & $-C 11 B$ & $-\mathrm{H} 11 \mathrm{E}$ & 109.39 \\
\hline H8BA & $-C 8 B$ & $-\mathrm{H} 8 \mathrm{BB}$ & 108.59 & $\mathrm{C} 10 \mathrm{~B}$ & $-C 11 B$ & $-\mathrm{H} 11 \mathrm{~F}$ & 109.32 \\
\hline $\mathrm{C} 10 \mathrm{~B}$ & $-C 9 B$ & $-\mathrm{H} 9 \mathrm{D}$ & 109.38 & $\mathrm{H} 11 \mathrm{D}$ & $-C 11 B$ & $-\mathrm{H} 11 \mathrm{E}$ & 109.64 \\
\hline $\mathrm{C} 10 \mathrm{~B}$ & $-C 9 B$ & $-\mathrm{H} 9 \mathrm{E}$ & 109.50 & $\mathrm{H} 11 \mathrm{D}$ & $-C 11 B$ & $-\mathrm{H} 11 \mathrm{~F}$ & 109.54 \\
\hline $\mathrm{C} 10 \mathrm{~B}$ & $-C 9 B$ & $-\mathrm{H} 9 \mathrm{~F}$ & 109.48 & $\mathrm{H} 11 \mathrm{E}$ & $-C 11 B$ & $-\mathrm{H} 11 \mathrm{~F}$ & 109.48 \\
\hline
\end{tabular}


Table S7 - Torsion Angles (Degrees)
for: 5

O1A - MO1A -O3A - C1A $-46.4(14)$

$\begin{array}{lllll}\text { N1A } & -M 01 A & -O 3 A & -\mathrm{C} 1 \mathrm{~A} & 36.7(14)\end{array}$

O1A_a -Mo1A -O3A - C1A $-152.8(14)$

O3A_a -Mo1A -O3A - C1A $80.1(17)$

N1A_a -Mo1A -O3A - - $1 A$ - $122.4(14)$

$\begin{array}{lllll}\mathrm{O} A \mathrm{~A}-\mathrm{MO} A & -\mathrm{N} 1 \mathrm{~A} & -\mathrm{C} 7 \mathrm{~A} & 78.7(12)\end{array}$

O1A - MO1A $-\mathrm{N} 1 \mathrm{~A}-\mathrm{C}-\mathrm{COA}-90.1(11)$

O3A - Mo1A $-\mathrm{N} 1 \mathrm{~A}-\mathrm{C}$ A $-21.9(11)$

O3A -Mo1A -N1A - C10A $169.2(11)$

O3A_a -Mo1A -N1A -C7A

O3A_a -Mo1A -N1A -C10A

N1A_a -Mo1A -N1A -C7A

N1A_a -Mo1A -N1A -

O3B - Mo1B $-\mathrm{N} 1 \mathrm{~B}-\mathrm{C}-\mathrm{COB}-166.2(12)$

O1B_b -Mo1B -N1B - - $7 B \quad-72.2(12)$

O1B_b -Mo1B -N1B -

O3B_b -Mo1B -N1B - - 7B

O3B_b - Mo1B -N1B -

N1B_b -Mo1B -N1B -

N1B_b -Mo1B -N1B -

$\begin{array}{lllll}\mathrm{O} 3 \mathrm{~B}-\mathrm{M} 1 \mathrm{~B} & -\mathrm{N} 1 \mathrm{~B} & -\mathrm{C} 7 \mathrm{~B} & 26.4(12)\end{array}$

O1B_b -Mo1B -O3B -

O1B - Mo1B - O3B - - $1 B \quad 151.3(14)$

$\mathrm{N1B}-\mathrm{Mo1B}-\mathrm{O} B \mathrm{~B}-\mathrm{C} 1 \mathrm{~B}-38.8(14)$

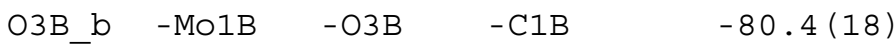

N1B_b - Mo1B - -O3B - - $1 B \quad-121.6(14)$

$\begin{array}{lllll}\mathrm{C} 8 \mathrm{~A}-\mathrm{O} 2 \mathrm{~A} & -\mathrm{C} 7 \mathrm{~A} & -\mathrm{N} 1 \mathrm{~A} & 5.2(17)\end{array}$ 


\begin{tabular}{|c|c|c|c|c|}
\hline C7A & $-02 \mathrm{~A}$ & $-C 8 A$ & $-\mathrm{C} 10 \mathrm{~A}$ & $-7.0(15)$ \\
\hline C8A & $-02 \mathrm{~A}$ & $-C 7 A$ & $-C 6 A$ & $-175.5(12)$ \\
\hline Mo1A & $-03 A$ & $-C 1 A$ & $-\mathrm{C} 2 \mathrm{~A}$ & $150.8(11)$ \\
\hline Mo1A & $-03 A$ & $-C 1 A$ & $-\mathrm{C} 6 \mathrm{~A}$ & $-35(2)$ \\
\hline $\mathrm{C} 8 \mathrm{~B}$ & $-02 B$ & $-C 7 B$ & $-\mathrm{C} 6 \mathrm{~B}$ & $-176.0(13)$ \\
\hline C7B & $-02 B$ & $-\mathrm{C} 8 \mathrm{~B}$ & $-\mathrm{C} 10 \mathrm{~B}$ & $-5.8(17)$ \\
\hline $\mathrm{C} 8 \mathrm{~B}$ & $-02 B$ & $-C 7 B$ & $-N 1 B$ & 2.1 (19) \\
\hline Mo1B & $-03 B$ & $-\mathrm{C} 1 \mathrm{~B}$ & $-\mathrm{C} 2 \mathrm{~B}$ & $-143.0(12)$ \\
\hline Mo1B & $-03 \mathrm{~B}$ & $-\mathrm{C} 1 \mathrm{~B}$ & $-\mathrm{C} 6 \mathrm{~B}$ & $33(2)$ \\
\hline C10A & $-\mathrm{N} 1 \mathrm{~A}$ & $-C 7 A$ & $-\mathrm{C} 6 \mathrm{~A}$ & $179.9(13)$ \\
\hline C10A & $-\mathrm{N} 1 \mathrm{~A}$ & $-C 7 A$ & $-02 \mathrm{~A}$ & $-0.9(17)$ \\
\hline C7A & $-\mathrm{N} 1 \mathrm{~A}$ & $-\mathrm{C} 10 \mathrm{~A}$ & $-C 8 A$ & $-3.6(15)$ \\
\hline Mo1A & $-\mathrm{N} 1 \mathrm{~A}$ & $-C 7 A$ & $-C 6 A$ & $9(2)$ \\
\hline Mo1A & $-\mathrm{N} 1 \mathrm{~A}$ & $-\mathrm{C} 10 \mathrm{~A}$ & $-\mathrm{C} 11 \mathrm{~A}$ & $51.1(15)$ \\
\hline Mo1A & $-\mathrm{N} 1 \mathrm{~A}$ & $-\mathrm{C} 10 \mathrm{~A}$ & $-C 8 A$ & $166.8(9)$ \\
\hline C7A & $-\mathrm{N} 1 \mathrm{~A}$ & $-\mathrm{C} 10 \mathrm{~A}$ & $-\mathrm{C} 9 \mathrm{~A}$ & $113.9(13)$ \\
\hline C7A & $-\mathrm{N} 1 \mathrm{~A}$ & $-\mathrm{C} 10 \mathrm{~A}$ & $-\mathrm{C} 11 \mathrm{~A}$ & $-119.3(13)$ \\
\hline Mo1A & $-\mathrm{N} 1 \mathrm{~A}$ & $-\mathrm{C} 10 \mathrm{~A}$ & $-C 9 A$ & $-75.7(14)$ \\
\hline Mo1A & $-\mathrm{N} 1 \mathrm{~A}$ & $-C 7 A$ & $-02 \mathrm{~A}$ & $-171.5(9)$ \\
\hline C7B & $-N 1 B$ & $-\mathrm{C} 10 \mathrm{~B}$ & $-\mathrm{C} 11 \mathrm{~B}$ & $-122.9(16)$ \\
\hline Mo1B & $-\mathrm{N} 1 \mathrm{~B}$ & $-\mathrm{C} 10 \mathrm{~B}$ & $-\mathrm{C} 9 \mathrm{~B}$ & $-58.8(17)$ \\
\hline Mo1B & $-N 1 B$ & $-\mathrm{C} 10 \mathrm{~B}$ & $-C 8 B$ & $-174.8(10)$ \\
\hline $\mathrm{C} 10 \mathrm{~B}$ & $-\mathrm{N} 1 \mathrm{~B}$ & $-C 7 B$ & $-02 B$ & $2.6(18)$ \\
\hline $\mathrm{C} 10 \mathrm{~B}$ & $-N 1 B$ & $-C 7 B$ & $-\mathrm{C} 6 \mathrm{~B}$ & $-179.6(14)$ \\
\hline C7B & $-N 1 B$ & $-\mathrm{C} 10 \mathrm{~B}$ & $-C 9 B$ & $110.2(15)$ \\
\hline Mo1B & $-N 1 B$ & $-\mathrm{C} 7 \mathrm{~B}$ & $-\mathrm{C} 6 \mathrm{~B}$ & $-10(2)$ \\
\hline C7B & $-\mathrm{N} 1 \mathrm{~B}$ & $-\mathrm{C} 10 \mathrm{~B}$ & $-C 8 B$ & $-5.8(16)$ \\
\hline Mo1B & $-\mathrm{N} 1 \mathrm{~B}$ & $-C 7 B$ & $-02 B$ & $172.6(10)$ \\
\hline
\end{tabular}




\begin{tabular}{|c|c|c|c|c|}
\hline Mo1B & $-N 1 B$ & $-\mathrm{C} 10 \mathrm{~B}$ & $-C 11 B$ & $68.1(17)$ \\
\hline $\mathrm{C} 2 \mathrm{~A}$ & $-\mathrm{C} 1 \mathrm{~A}$ & $-\mathrm{C} 6 \mathrm{~A}$ & $-\mathrm{C} 5 \mathrm{~A}$ & $-3(2)$ \\
\hline C6A & $-\mathrm{C} 1 \mathrm{~A}$ & $-\mathrm{C} 2 \mathrm{~A}$ & $-C 3 A$ & $3(2)$ \\
\hline O3A & $-\mathrm{C} 1 \mathrm{~A}$ & $-\mathrm{C} 6 \mathrm{~A}$ & $-C 7 A$ & $2(2)$ \\
\hline O3A & $-\mathrm{C} 1 \mathrm{~A}$ & $-\mathrm{C} 2 \mathrm{~A}$ & $-C 3 A$ & $178.2(14)$ \\
\hline $\mathrm{C} 2 \mathrm{~A}$ & $-\mathrm{C} 1 \mathrm{~A}$ & $-C 6 A$ & $-C 7 A$ & $176.6(12)$ \\
\hline O3A & $-\mathrm{C} 1 \mathrm{~A}$ & $-C 6 A$ & $-C 5 A$ & $-177.3(14)$ \\
\hline $\mathrm{C} 1 \mathrm{~A}$ & $-\mathrm{C} 2 \mathrm{~A}$ & $-C 3 A$ & $-\mathrm{C} 4 \mathrm{~A}$ & $-2(3)$ \\
\hline $\mathrm{C} 2 \mathrm{~A}$ & $-C 3 A$ & $-\mathrm{C} 4 \mathrm{~A}$ & $-C 5 A$ & $0(3)$ \\
\hline C3A & $-C 4 A$ & $-\mathrm{C} 5 \mathrm{~A}$ & $-\mathrm{C} 6 \mathrm{~A}$ & $1(3)$ \\
\hline $\mathrm{C} 4 \mathrm{~A}$ & $-\mathrm{C} 5 \mathrm{~A}$ & $-\mathrm{C} 6 \mathrm{~A}$ & $-C 1 A$ & $1(2)$ \\
\hline $\mathrm{C} 4 \mathrm{~A}$ & $-\mathrm{C} 5 \mathrm{~A}$ & $-\mathrm{C} 6 \mathrm{~A}$ & $-\mathrm{C} 7 \mathrm{~A}$ & $-178.6(15)$ \\
\hline C5A & $-\mathrm{C} 6 \mathrm{~A}$ & $-C 7 A$ & $-\mathrm{N} 1 \mathrm{~A}$ & $-175.1(14)$ \\
\hline C5A & $-\mathrm{C} 6 \mathrm{~A}$ & $-\mathrm{C} 7 \mathrm{~A}$ & $-02 \mathrm{~A}$ & $5.7(19)$ \\
\hline $\mathrm{C} 1 \mathrm{~A}$ & $-\mathrm{C} 6 \mathrm{~A}$ & $-\mathrm{C} 7 \mathrm{~A}$ & $-02 \mathrm{~A}$ & $-173.5(12)$ \\
\hline $\mathrm{C} 1 \mathrm{~A}$ & $-\mathrm{C} 6 \mathrm{~A}$ & $-\mathrm{C} 7 \mathrm{~A}$ & $-N 1 A$ & $6(2)$ \\
\hline $\mathrm{O} 2 \mathrm{~A}$ & $-\mathrm{C} 8 \mathrm{~A}$ & $-\mathrm{C} 10 \mathrm{~A}$ & $-\mathrm{N} 1 \mathrm{~A}$ & $6.3(15)$ \\
\hline $\mathrm{O} 2 \mathrm{~A}$ & $-C 8 A$ & - C10A & $-C 9 A$ & $-109.6(15)$ \\
\hline $\mathrm{O} 2 \mathrm{~A}$ & $-C 8 A$ & $-\mathrm{C} 10 \mathrm{~A}$ & $-C 11 A$ & $123.9(14)$ \\
\hline $\mathrm{O} 3 \mathrm{~B}$ & $-C 1 B$ & $-\mathrm{C} 6 \mathrm{~B}$ & $-C 5 B$ & $177.8(15)$ \\
\hline $\mathrm{O} 3 \mathrm{~B}$ & $-C 1 B$ & $-\mathrm{C} 2 \mathrm{~B}$ & $-C 3 B$ & $-179.6(15)$ \\
\hline $\mathrm{C} 6 \mathrm{~B}$ & $-C 1 B$ & $-\mathrm{C} 2 \mathrm{~B}$ & $-C 3 B$ & $4(2)$ \\
\hline $\mathrm{C} 2 \mathrm{~B}$ & $-\mathrm{C} 1 \mathrm{~B}$ & $-\mathrm{C} 6 \mathrm{~B}$ & $-C 7 B$ & $179.6(13)$ \\
\hline $\mathrm{C} 2 \mathrm{~B}$ & $-C 1 B$ & $-C 6 B$ & $-C 5 B$ & $-6(2)$ \\
\hline O3B & $-C 1 B$ & $-\mathrm{C} 6 \mathrm{~B}$ & $-C 7 B$ & $3(2)$ \\
\hline $\mathrm{C} 1 \mathrm{~B}$ & $-\mathrm{C} 2 \mathrm{~B}$ & $-C 3 B$ & $-C 4 B$ & $-2(3)$ \\
\hline $\mathrm{C} 2 \mathrm{~B}$ & $-C 3 B$ & $-C 4 B$ & $-C 5 B$ & $2(3)$ \\
\hline C3B & $-C 4 B$ & $-C 5 B$ & $-\mathrm{C} 6 \mathrm{~B}$ & $-4(3)$ \\
\hline $\mathrm{C} 4 \mathrm{~B}$ & $-C 5 B$ & $-\mathrm{C} 6 \mathrm{~B}$ & $-C 7 B$ & $-179.4(16)$ \\
\hline
\end{tabular}




$\begin{array}{llllr}\mathrm{C} 4 \mathrm{~B} & -\mathrm{C} 5 \mathrm{~B} & -\mathrm{C} 6 \mathrm{~B} & -\mathrm{C} 1 \mathrm{~B} & 6(3) \\ \mathrm{C} 5 \mathrm{~B} & -\mathrm{C} 6 \mathrm{~B} & -\mathrm{C} 7 \mathrm{~B} & -\mathrm{O} 2 \mathrm{~B} & -7(2) \\ \mathrm{C} 5 \mathrm{~B} & -\mathrm{C} 6 \mathrm{~B} & -\mathrm{C} 7 \mathrm{~B} & -\mathrm{N} 1 \mathrm{~B} & 174.9(16) \\ \mathrm{C} 1 \mathrm{~B} & -\mathrm{C} 6 \mathrm{~B} & -\mathrm{C} 7 \mathrm{~B} & -\mathrm{O} 2 \mathrm{~B} & 167.3(13) \\ \mathrm{C} 1 \mathrm{~B} & -\mathrm{C} 6 \mathrm{~B} & -\mathrm{C} 7 \mathrm{~B} & -\mathrm{N} 1 \mathrm{~B} & -11(2) \\ \mathrm{O} 2 \mathrm{~B} & -\mathrm{C} 8 \mathrm{~B} & -\mathrm{C} 10 \mathrm{~B} & -\mathrm{C} 11 \mathrm{~B} & 126.9(15) \\ \mathrm{O} 2 \mathrm{~B} & -\mathrm{C} 8 \mathrm{~B} & -\mathrm{C} 10 \mathrm{~B} & -\mathrm{N} 1 \mathrm{~B} & 7.0(17) \\ \text { O2B } & -\mathrm{C} 8 \mathrm{~B} & -\mathrm{C} 10 \mathrm{~B} & -\mathrm{C} 9 \mathrm{~B} & -108.8(17)\end{array}$



$\begin{aligned} \text { Table s8 - } & \text { Contact Distances (Angstrom) } \\ & \text { for: } 5\end{aligned}$

\begin{tabular}{|c|c|c|c|c|c|}
\hline $\operatorname{Mo1A}$ &. $\mathrm{C} 5 \mathrm{~B}$ & $4.098(19)$ & O3B & .H9F_b & 2.3790 \\
\hline Mo1A & . C5B_C & $4.098(19)$ & $\mathrm{C} 1 \mathrm{~B}$ & . $C 11 B \_b$ & $3.59(2)$ \\
\hline Mo1A & . H5BA & 3.3769 & $\mathrm{C} 2 \mathrm{~B}$ & . C9A_m & $3.55(2)$ \\
\hline Mo1A & . H11A & 3.4467 & $\mathrm{C} 3 \mathrm{~B}$ & . C9A_m & $3.41(3)$ \\
\hline Mo1A & . H9A & 3.6485 & $\mathrm{C} 3 \mathrm{~B}$ & $.02 A_{-} d$ & $3.40(2)$ \\
\hline Mo1A & .H9A_a & 3.6485 & $\mathrm{C} 4 \mathrm{~B}$ & . C8A_d & $3.58(3)$ \\
\hline Mo1A & .H5BA_C & 3.3769 & $\mathrm{C} 4 \mathrm{~B}$ & $.02 \mathrm{~A} \_d$ & $3.20(2)$ \\
\hline Mo1A & .H11A_a & 3.4467 & C5B & Mo1A & $4.098(19)$ \\
\hline Mo1B & .H9F_b & 3.4699 & C5B & . Mo1A & $4.098(19)$ \\
\hline Mo1B &. $\mathrm{H} 9 \mathrm{~F}$ & 3.4699 & C8A &. $\mathrm{C}_{4} \mathrm{~B}_{-} \mathrm{f}$ & $3.58(3)$ \\
\hline $\mathrm{O} 2 \mathrm{~A}$ & . $\mathrm{C} 4 \mathrm{~B} \_\mathrm{f}$ & $3.20(2)$ & C9A &.$C 3 B \_g$ & $3.41(3)$ \\
\hline $\mathrm{O} 2 \mathrm{~A}$ &.$C 3 B_{-} \mathrm{f}$ & $3.40(2)$ & C9A & . C2B_g & $3.55(2)$ \\
\hline O3A & . C11A_a & $3.05(2)$ & C9A & $.03 A_{2} a$ & $3.35(2)$ \\
\hline O3A & . C9A_a & $3.35(2)$ & $\mathrm{C} 9 \mathrm{~B}$ & $.03 B \_b$ & $3.13(2)$ \\
\hline $\mathrm{O} 3 \mathrm{~B}$ & . C11B_b & $3.32(2)$ & $\mathrm{C} 11 \mathrm{~A}$ & $.03 A_{-}$a & $3.05(2)$ \\
\hline O3B & . C9B_b & $3.13(2)$ & $\mathrm{C} 11 \mathrm{~B}$ & . $\mathrm{C} 1 \mathrm{~B} \_\mathrm{b}$ & $3.59(2)$ \\
\hline $\mathrm{O} 1 \mathrm{~A}$ & . $\mathrm{H} 4 \mathrm{BA}$ & 2.7954 & $\mathrm{C} 11 \mathrm{~B}$ & $.03 B \_b$ & $3.32(2)$ \\
\hline $\mathrm{O} A \mathrm{~A}$ & .H5AA_d & 2.5160 & $\mathrm{C} 1 \mathrm{~A}$ & .H9A_a & 2.7790 \\
\hline $\mathrm{O} 1 \mathrm{~A}$ & .H5BA_C & 2.6496 & $\mathrm{C} 1 \mathrm{~A}$ & .H11A_a & 2.9937 \\
\hline O1B & . $\mathrm{H} 8 \mathrm{BB} \mathrm{k}$ & 2.8958 & C1B &. $\mathrm{H} 11 \mathrm{D}$ b & 2.6977 \\
\hline $\mathrm{O} 2 \mathrm{~A}$ & . $\mathrm{H} 3 \mathrm{BA} \mathrm{e}$ & 2.7196 & $\mathrm{C} 2 \mathrm{~B}$ &. $\mathrm{H} 11 \mathrm{C} d$ & 2.7363 \\
\hline $\mathrm{O} 2 \mathrm{~A}$ & . H5AA & 2.3680 & C3A &. $\mathrm{H} 8 \mathrm{AA} \mathrm{d}$ & 2.9751 \\
\hline $\mathrm{O} 2 \mathrm{~B}$ &. $\mathrm{H} 4 \mathrm{AA} \quad 1$ & 2.6142 & $\mathrm{C} 4 \mathrm{~A}$ &. $\mathrm{H} 8 \mathrm{AA} \mathrm{d}$ & 3.0073 \\
\hline $\mathrm{O} 2 \mathrm{~B}$ & . H2AA & 2.7358 & $\mathrm{C} 4 \mathrm{~B}$ & .H8AB_d & 3.0119 \\
\hline $\mathrm{O} 2 \mathrm{~B}$ & . H5BA & 2.3675 & C5A &. $\mathrm{H} 2 \mathrm{BA} \mathrm{e}$ & 2.9433 \\
\hline O3A & .H9A_a & 2.6721 & C5B & .H8AB_d & 3.0746 \\
\hline O3A & .H11A_a & 2.2907 & C6A & .H9A_a & 2.9116 \\
\hline
\end{tabular}




\begin{tabular}{|c|c|c|c|c|c|}
\hline O3B & .H11D_b & 2.6413 & $\mathrm{C} 6 \mathrm{~B}$ & .H11D_b & 2.9378 \\
\hline C7A & .H9A_a & 2.9777 & H9C &. $\mathrm{H} 8 \mathrm{AA}$ & 2.4477 \\
\hline $\mathrm{C} 7 \mathrm{~B}$ & .H11D_b & 3.0136 & $\mathrm{H} 9 \mathrm{C}$ &. $\mathrm{H} 11 \mathrm{~B}$ & 2.5984 \\
\hline $\mathrm{C} 7 \mathrm{~B}$ & . H2AA & 2.9751 & H9D &.$C 7 B$ & 3.0624 \\
\hline $\mathrm{C} 7 \mathrm{~B}$ & .H9D & 3.0624 & H9D & . H8BA & 2.4179 \\
\hline $\mathrm{H} 2 \mathrm{AA}$ & $.02 B$ & 2.7358 & $\mathrm{H} 9 \mathrm{E}$ &. $\mathrm{H} 11 \mathrm{E}$ & 2.5475 \\
\hline $\mathrm{H} 2 \mathrm{AA}$ &. $\mathrm{C} 7 \mathrm{~B}$ & 2.9751 & $\mathrm{H} 9 \mathrm{E}$ & . H8BA & 2.3939 \\
\hline $\mathrm{H} 4 \mathrm{AA}$ & $.02 B \_h$ & 2.6142 & $\mathrm{H} 9 \mathrm{~F}$ & $.03 B \_b$ & 2.3790 \\
\hline $\mathrm{H} 5 \mathrm{AA}$ & $.02 \mathrm{~A}$ & 2.3680 & $\mathrm{H} 9 \mathrm{~F}$ & .H11D & 2.5399 \\
\hline H5AA & $.01 A_{-} i$ & 2.5160 & $\mathrm{H} 9 \mathrm{~F}$ & . Mo1B & 3.4699 \\
\hline $\mathrm{H} 8 \mathrm{AA}$ &. $\mathrm{H} 9 \mathrm{C}$ & 2.4477 & H5BA & Mo1A & 3.3769 \\
\hline H8AA & .C3A_i & 2.9751 & H5BA & Mo1A & 3.3769 \\
\hline H8AA & .H9B & 2.4686 & H5BA & $.02 \mathrm{~B}$ & 2.3675 \\
\hline H8AA & . C4A_i & 3.0073 & H5BA & .01A_a & 2.6496 \\
\hline $\mathrm{H} 8 \mathrm{AB}$ & . H11B & 2.4236 & H8BA &. $\mathrm{H} 9 \mathrm{E}$ & 2.3939 \\
\hline $\mathrm{H} 8 \mathrm{AB}$ &. $\mathrm{H} 11 \mathrm{C}$ & 2.3519 & H8BA &. $\mathrm{H} 9 \mathrm{D}$ & 2.4179 \\
\hline H8AB & . $\mathrm{C} 5 \mathrm{~B} \_\mathrm{f}$ & 3.0746 & $\mathrm{H} 11 \mathrm{~A}$ & . Mo1A & 3.4467 \\
\hline H8AB & . $\mathrm{C} 4 \mathrm{~B} \_\mathrm{f}$ & 3.0119 & $\mathrm{H} 11 \mathrm{~A}$ & . C1A_a & 2.9937 \\
\hline $\mathrm{H} 2 \mathrm{BA}$ & . C5A_n & 2.9433 & $\mathrm{H} 11 \mathrm{~A}$ &. $\mathrm{H} 9 \mathrm{~A}$ & 2.5369 \\
\hline H3 BA & $.02 A_{-} n$ & 2.7196 & $\mathrm{H} 11 \mathrm{~A}$ & .03A_a & 2.2907 \\
\hline $\mathrm{H} 4 \mathrm{BA}$ & $.01 \mathrm{~A}$ & 2.7954 & $\mathrm{H} 11 \mathrm{~B}$ &.$H 11 E \_j$ & 2.5360 \\
\hline $\mathrm{H} 9 \mathrm{~A}$ & . Mo1A & 3.6485 & $\mathrm{H} 11 \mathrm{~B}$ & . $\mathrm{H} 8 \mathrm{AB}$ & 2.4236 \\
\hline H9A & $.03 A_{2} a$ & 2.6721 & $\mathrm{H} 11 \mathrm{~B}$ &. $\mathrm{H} 9 \mathrm{C}$ & 2.5984 \\
\hline H9A & . C1A_a & 2.7790 & $\mathrm{H} 11 \mathrm{C}$ & . $\mathrm{C} 2 \mathrm{~B} \_\mathrm{f}$ & 2.7363 \\
\hline H9A & . H11A & 2.5369 & $\mathrm{H} 11 \mathrm{C}$ &. $\mathrm{H} 8 \mathrm{AB}$ & 2.3519 \\
\hline H9A & . C7A_a & 2.9777 & $\mathrm{H} 11 \mathrm{D}$ &. $\mathrm{H} 9 \mathrm{~F}$ & 2.5399 \\
\hline H9A & . C6A_a & 2.9116 & $\mathrm{H} 11 \mathrm{D}$ & $.03 B \_b$ & 2.6413 \\
\hline H9B & .H9B_a & 2.4944 & $\mathrm{H} 11 \mathrm{D}$ & . $\mathrm{C} 1 \mathrm{~B} \_\mathrm{b}$ & 2.6977 \\
\hline
\end{tabular}




$\begin{array}{llllll}\text { H9B } & . \text { H8AA } & 2.4686 & \text { H11D } & . \text { C6B_b } & 2.9378 \\ \text { H11D } & \text {.C7B_b } & 3.0137 & \text { H11F } & . \text { H11F_b } & 2.4103 \\ \text { H11E } & . \text { H9E } & 2.5475 & \text { H8BB } & . \text { H11E } & 2.4361 \\ \text { H11E } & . \text { H8BB } & 2.4361 & \text { H8BB } & . \text { H11F } & 2.3619 \\ \text { H11E } & . \text {.H11B_O } & 2.5360 & \text { H8BB } & .01 B \_p & 2.8958 \\ \text { H11F } & . \text {.H8BB } & 2.3619 & & & \end{array}$


Table S9 - Hydrogen Bonds (Angstrom, Deg) for: 5

\begin{tabular}{|c|c|c|c|c|c|c|c|}
\hline C5A & $--\mathrm{H} 5 \mathrm{AA}$ & . $02 \mathrm{~A}$ & 0.9300 & 2.3700 & $2.71(2)$ & 101.00 & . \\
\hline C5A & - - H5AA & . O1A & 0.9300 & 2.5200 & $3.43(2)$ & 169.00 & 7_466 \\
\hline C9B & $--\mathrm{H} 9 \mathrm{~F}$ & . O3B & 0.9600 & 2.3800 & $3.13(2)$ & 135.00 & $4 \_756$ \\
\hline C5B & - - H5BA & . $\mathrm{O} 2 \mathrm{~B}$ & 0.9300 & 2.3700 & $2.71(2)$ & 101.00 & . \\
\hline $\mathrm{C} 11 \mathrm{~A}$ & $--\mathrm{H} 11 \mathrm{~A}$ & . O3A & 0.9600 & 2.2900 & $3.05(2)$ & 135.00 & $3-^{576}$ \\
\hline
\end{tabular}


Translation of Symmetry Code to Equiv.Pos

\begin{tabular}{|c|c|c|}
\hline [ & 3576.00 & $=x, 2-y, 1-z$ \\
\hline$=[$ & 4756.00 & $=2-x, y, 3 / 2-z$ \\
\hline$=[$ & 7566.00 & $=1 / 2+x, 3 / 2-y, 1-z$ \\
\hline$=[$ & 1455.00 & $=-1+x, y, z$ \\
\hline$=\mathrm{l}$ & 7466.00 & $=-1 / 2+x, 3 / 2-y, 1-z$ \\
\hline$=[$ & 3476.00 & $=-1+x, 2-y, 1-z$ \\
\hline$=[$ & 5445.00 & $=-1 / 2+x,-1 / 2+y, z$ \\
\hline & 7466.00 & $=-1 / 2+x, 3 / 2-y, 1-z$ \\
\hline & 2674.00 & $=1-x, 2-y,-1 / 2+z$ \\
\hline$=[$ & 5545.00 & $=1 / 2+x,-1 / 2+y, z$ \\
\hline$=[$ & 5555.00 & $=1 / 2+x, 1 / 2+y, z$ \\
\hline$-\mathrm{L}$ & 3676.00 & $=1+x, 2-y, 1-z$ \\
\hline$L$ & 1655.00 & $=1+x, y, z$ \\
\hline$=$ & 2675.00 & $=1-x, 2-y, 1 / 2$ \\
\hline & 5455.00 & $=-1 / 2+x, 1 / 2+y, z$ \\
\hline
\end{tabular}



Table S1 - Crystal Data and Details of the structure Determination
for: 8

\section{Crystal Data}

Formula $\mathrm{C} 20 \mathrm{H} 20 \mathrm{~N} 2 \mathrm{O} 5 \mathrm{~V}$

Formula Weight 419.32

Crystal system

Orthorhombic Space group P212121

(No. 19)

$a, b, c$ [Angstrom]

$8.8894(6)$

$11.7764(9)$

$18.1078(14)$

$\mathrm{V}$ [Ang**3]

$1895.6(2)$

Z

$\mathrm{D}(\mathrm{calc}) \quad[\mathrm{g} / \mathrm{cm} * * 3]$

1.469

$\mathrm{Mu}(\mathrm{MoKa}) \quad[/ \mathrm{mm}]$

0.558

$F(000)$

868

Crystal size [mm]

Data Collection

Temperature (K)

293

Radiation [Angstrom]

MoKa

0.71073

Theta Min-Max [Deg]

$2.1,28.3$

Dataset

$-11: 11 ;-15: 14 ;-23: 21$

Tot., Uniq. Data, R(int)

14681, $4640, \quad 0.094$

Observed data $[I>2.0$ sigma (I)]

1776

Refinement

Nref, Npar

$4640, \quad 309$

R， WR2， S

$0.0470,0.0624,0.81$

$\mathrm{w}=1 /\left[\backslash \mathrm{S}^{\wedge} 2^{\wedge}\left(\mathrm{FO} \mathrm{O}^{\wedge} 2^{\wedge}\right)+(0.0104 \mathrm{P})^{\wedge} 2^{\wedge}\right]$

where $\mathrm{P}=\left(\mathrm{FO}^{\wedge} 2^{\wedge}+2 \mathrm{FC}^{\wedge} 2^{\wedge}\right) / 3$

Max. and Av. Shift/Error

$0.01,0.00$

Flack $\mathrm{x}$

$0.54(2)$

Min. and Max. Resd. Dens. [e/Ang^3]

$-0.22,0.50$ 
Table S2 - Final Coordinates and Equivalent Isotropic Displacement Parameters of the non-Hydrogen atoms

for: 8

\begin{tabular}{|c|c|c|c|c|}
\hline Atom & $\mathrm{x}$ & Y & $z$ & $\mathrm{U}(\mathrm{eq}) \quad\left[\mathrm{Ang}^{\wedge} 2\right]$ \\
\hline---- & --- & --- & --- & ---------- \\
\hline V & $0.13107(7)$ & $1.09997(5)$ & $0.38738(3)$ & $0.0523(3)$ \\
\hline O & $-0.0459(3)$ & $1.1072(2)$ & $0.37660(11)$ & $0.0706(10)$ \\
\hline O1A & $0.1977(3)$ & $1.0312(2)$ & $0.47537(12)$ & $0.0698(10)$ \\
\hline O1B & $0.2495(3)$ & $1.1604(2)$ & $0.31063(12)$ & $0.0594(11)$ \\
\hline $\mathrm{O} 2 \mathrm{~A}$ & $0.2294(3)$ & $0.7525(2)$ & $0.32931(16)$ & $0.0703(12)$ \\
\hline $\mathrm{O} 2 \mathrm{~B}$ & $0.2253(3)$ & $1.4443(2)$ & $0.45306(16)$ & $0.0747(12)$ \\
\hline $\mathrm{N} 1 \mathrm{~A}$ & $0.1619(3)$ & $0.9428(3)$ & $0.33643(16)$ & $0.0530(14)$ \\
\hline N1B & $0.1603(4)$ & $1.2541(3)$ & $0.44151(17)$ & $0.0607(14)$ \\
\hline $\mathrm{C} 1 \mathrm{~A}$ & $0.2554(4)$ & $0.9313(3)$ & $0.4913(2)$ & $0.0503(17)$ \\
\hline $\mathrm{C} 1 \mathrm{~B}$ & $0.3024(4)$ & $1.2632(3)$ & 0.2955 ( 2 ) & $0.0473(17)$ \\
\hline $\mathrm{C} 2 \mathrm{~A}$ & $0.3042(4)$ & $0.9145(4)$ & $0.5640(2)$ & $0.0703(19)$ \\
\hline $\mathrm{C} 2 \mathrm{~B}$ & $0.3701(4)$ & $1.2786(3)$ & 0.22627 (19) & $0.0557(17)$ \\
\hline C3A & $0.3680(5)$ & $0.8137(4)$ & $0.5841(2)$ & $0.0760(17)$ \\
\hline C3B & $0.4245(4)$ & $1.3835(4)$ & $0.2067(2)$ & $0.0647(17)$ \\
\hline $\mathrm{C} 4 \mathrm{~A}$ & $0.3827(5)$ & $0.7256(4)$ & $0.5358(3)$ & $0.0760(17)$ \\
\hline C4B & $0.4188(5)$ & $1.4735(4)$ & $0.2546(3)$ & $0.080(2)$ \\
\hline C5A & $0.3347(4)$ & $0.7414(3)$ & $0.4645(2)$ & $0.0660(17)$ \\
\hline C5B & $0.3512(5)$ & $1.4586(4)$ & $0.3220(2)$ & 0.0777 (19) \\
\hline $\mathrm{C} 6 \mathrm{~A}$ & $0.2711(4)$ & $0.8419(4)$ & $0.4411(2)$ & $0.0443(17)$ \\
\hline $\mathrm{C} 6 \mathrm{~B}$ & $0.2912(4)$ & $1.3549(3)$ & $0.3440(2)$ & $0.0493(17)$ \\
\hline C7A & $0.2176(4)$ & $0.8523(4)$ & $0.36463(19)$ & $0.0483(17)$ \\
\hline C7B & $0.2193(5)$ & $1.3454(4)$ & $0.4175(2)$ & $0.0517(17)$ \\
\hline$\star C 8 A$ & 0.1619 (19) & $0.7380(13)$ & $0.2593(10)$ & $0.067(5)$ \\
\hline$\star \mathrm{C} 8 \mathrm{~B}$ & $0.162(2)$ & $1.4603(12)$ & $0.5206(11)$ & $0.078(7)$ \\
\hline *C9A & $0.1686(19)$ & $0.8460(11)$ & $0.2180(8)$ & $0.074(6)$ \\
\hline
\end{tabular}




$\begin{array}{lcccc}{ }^{* \mathrm{C} 9 \mathrm{~B}} & 0.0459(12) & 1.3733(11) & 0.5360(7) & 0.078(5) \\ { } \mathrm{C} 10 \mathrm{~A} & 0.1043(19) & 0.9460(15) & 0.2556(12) & 0.087(8) \\ { }^{*} \mathrm{C} 10 \mathrm{~B} & 0.110(2) & 1.2608(11) & 0.5176(11) & 0.083(6) \\ { }^{*} \mathrm{C} 9 \mathrm{C} & 0.086(2) & 0.8292(16) & 0.2271(13) & 0.103(9) \\ { }^{*} \mathrm{C9D} & 0.118(3) & 1.3417(14) & 0.5617(9) & 0.084(8) \\ { }^{*} \mathrm{C} 10 \mathrm{C} & 0.117(3) & 0.9400(13) & 0.2622(15) & 0.085(11) \\ { }^{*} \mathrm{C} 10 \mathrm{D} & 0.058(3) & 1.254(2) & 0.5108(13) & 0.085(9) \\ { }^{*} \mathrm{C} 8 \mathrm{C} & 0.216(2) & 0.756(2) & 0.2447(12) & 0.090(10) \\ { }^{*} \mathrm{C} 8 \mathrm{D} & 0.143(3) & 1.4546(16) & 0.5283(14) & 0.059(9)\end{array}$

$U(e q)=1 / 3$ of the trace of the orthogonalized $U$ Tensor Starred Atom sites have a S.O.F less than 1.0 
Table S3 - Hydrogen Atom Positions and Isotropic Displacement Parameters

for: 8

\begin{tabular}{|c|c|c|c|c|c|}
\hline Atom & $\mathrm{x}$ & Y & z & U (iso) & {$\left[\mathrm{Ang}^{\wedge} 2\right]$} \\
\hline---- & --- & --- & --- & ----- & ----- \\
\hline *H8AA & 0.05790 & 0.71480 & 0.26530 & & 0.0810 \\
\hline $\mathrm{H} 2 \mathrm{AA}$ & 0.29310 & 0.97220 & 0.59870 & & 0.0850 \\
\hline H3AА & 0.40260 & 0.80490 & 0.63220 & & 0.0910 \\
\hline $\mathrm{H} 4 \mathrm{AA}$ & 0.42400 & 0.65680 & 0.55070 & & 0.0910 \\
\hline H5AA & 0.34540 & 0.68240 & 0.43080 & & 0.0790 \\
\hline * H9AB & 0.11630 & 0.83580 & 0.17150 & & 0.0890 \\
\hline $\mathrm{H} 2 \mathrm{BA}$ & 0.37810 & 1.21790 & 0.19360 & & 0.0670 \\
\hline H3 BA & 0.46600 & 1.39360 & 0.16010 & & 0.0770 \\
\hline $\mathrm{H} 4 \mathrm{BA}$ & 0.46000 & 1.54330 & 0.24170 & & 0.0960 \\
\hline H5BA & 0.34530 & 1.52000 & 0.35410 & & 0.0930 \\
\hline * H8BA & 0.11640 & 1.53520 & 0.52260 & & 0.0930 \\
\hline * $\mathrm{H} 8 \mathrm{BB}$ & 0.23930 & 1.45670 & 0.55830 & & 0.0930 \\
\hline *H9BA & -0.04290 & 1.38740 & 0.50620 & & 0.0930 \\
\hline *H9BB & 0.01720 & 1.37570 & 0.58760 & & 0.0930 \\
\hline *H8AB & 0.21430 & 0.67910 & 0.23210 & & 0.0810 \\
\hline * $\mathrm{H} 1 \mathrm{OB}$ & -0.00470 & 0.94300 & 0.25450 & & 0.1050 \\
\hline * $\mathrm{H} 10 \mathrm{C}$ & 0.13670 & 1.01530 & 0.23140 & & 0.1050 \\
\hline * $\mathrm{H} 10 \mathrm{~F}$ & 0.19500 & 1.24580 & 0.55000 & & 0.0990 \\
\hline *H1 OG & 0.03520 & 1.20270 & 0.52640 & & 0.0990 \\
\hline *H9AA & 0.27310 & 0.86200 & 0.20660 & & 0.0890 \\
\hline * $\mathrm{H} 8 \mathrm{CA}$ & 0.30690 & 0.78720 & 0.22310 & & 0.1070 \\
\hline * H8 CB & 0.20050 & 0.68030 & 0.22520 & & 0.1070 \\
\hline * $\mathrm{H} 9 \mathrm{CA}$ & 0.07500 & 0.83790 & 0.17410 & & 0.1230 \\
\hline *H9CB & -0.00620 & 0.79660 & 0.24670 & & 0.1230 \\
\hline * $\mathrm{H} 10 \mathrm{~A}$ & 0.19440 & 0.97720 & 0.23340 & & 0.1010 \\
\hline *H1 OD & -0.04510 & 1.27240 & 0.49700 & & 0.1020 \\
\hline
\end{tabular}




$\begin{array}{lllll}* \mathrm{H} 1 \mathrm{OE} & 0.05870 & 1.18040 & 0.53430 & 0.1020 \\ \text { * } \mathrm{H1OH} & 0.02660 & 0.98580 & 0.25780 & 0.1010 \\ \text { *H8DA } & 0.20310 & 1.50060 & 0.56160 & 0.0710 \\ \text { *H8DB } & 0.04730 & 1.49240 & 0.52120 & 0.0710 \\ \text { *H9DA } & 0.04790 & 1.35020 & 0.60260 & 0.1010 \\ \text { *H9DB } & 0.21210 & 1.31450 & 0.58190 & 0.1010\end{array}$

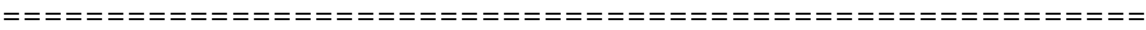

The Temperature Factor has the Form of $\operatorname{Exp}(-\mathrm{T})$ Where $\mathrm{T}=8 *(\mathrm{Pi} * 2) * \mathrm{U} *(\mathrm{Sin}(\mathrm{Theta}) /$ Lambda $) * * 2$ for Isotropic Atoms 


\begin{tabular}{|c|c|c|c|c|c|c|}
\hline Atom & $\mathrm{U}(1,1)$ or $\mathrm{U}$ & $\mathrm{J} \quad \mathrm{U}(2,2)$ & $\mathrm{U}(3,3)$ & $\mathrm{U}(2,3)$ & $\mathrm{U}(1,3)$ & $\mathrm{U}(1,2)$ \\
\hline--- & ----- & ----- & ----- & ----- & ----- & ----- \\
\hline V & $0.0702(5)$ & $0.0452(4)$ & $0.0415(4)$ & $0.0045(4)$ & $0.0035(4)$ & $0.0024(4)$ \\
\hline 0 & $0.0671(17)$ & $0.0743(16)$ & $0.0704(17)$ & $0.0109(18)$ & $-0.0020(14)$ & $0.0103(17)$ \\
\hline $\mathrm{O} 1 \mathrm{~A}$ & $0.118(2)$ & $0.0511(17)$ & $0.0403(17)$ & $0.0037(14)$ & $-0.0122(15)$ & $0.0207(17)$ \\
\hline $\mathrm{O} 1 \mathrm{~B}$ & $0.088(2)$ & $0.0408(17)$ & $0.0493(18)$ & $-0.0061(14)$ & $0.0155(14)$ & $-0.0165(15)$ \\
\hline $\mathrm{O} 2 \mathrm{~A}$ & $0.100(2)$ & $0.056(2)$ & $0.055(2)$ & $-0.0110(17)$ & $-0.0118(18)$ & $0.0073(18)$ \\
\hline $\mathrm{O} 2 \mathrm{~B}$ & $0.104(2)$ & $0.054(2)$ & $0.066(2)$ & $-0.0220(17)$ & $0.0171(17)$ & $-0.0048(17)$ \\
\hline $\mathrm{N} 1 \mathrm{~A}$ & $0.080(3)$ & $0.042(2)$ & $0.037(2)$ & $0.0052(16)$ & $-0.0072(19)$ & $-0.002(2)$ \\
\hline N1B & $0.089(3)$ & $0.044(2)$ & $0.049(2)$ & $0.0003(19)$ & $0.018(2)$ & $-0.005(2)$ \\
\hline $\mathrm{C} 1 \mathrm{~A}$ & $0.055(3)$ & $0.055(3)$ & $0.041(3)$ & $0.007(2)$ & $-0.003(2)$ & $-0.001(2)$ \\
\hline C1B & $0.058(3)$ & $0.042(3)$ & $0.042(3)$ & $0.006(2)$ & $-0.004(2)$ & $0.000(2)$ \\
\hline $\mathrm{C} 2 \mathrm{~A}$ & $0.109(4)$ & $0.063(3)$ & $0.039(3)$ & $0.010(2)$ & $-0.009(2)$ & $-0.001(3)$ \\
\hline $\mathrm{C} 2 \mathrm{~B}$ & $0.073(3)$ & $0.051(3)$ & $0.043(3)$ & $0.007(2)$ & $0.012(2)$ & $0.000(3)$ \\
\hline C3A & $0.089(3)$ & $0.088(3)$ & $0.051(3)$ & $0.021(3)$ & $-0.009(3)$ & $-0.001(3)$ \\
\hline C3B & $0.066(3)$ & $0.075(3)$ & $0.053(3)$ & $0.017(3)$ & $0.010(2)$ & $0.012(3)$ \\
\hline $\mathrm{C} 4 \mathrm{~A}$ & $0.084(3)$ & $0.066(3)$ & $0.078(3)$ & $0.014(3)$ & $-0.023(3)$ & $0.015(3)$ \\
\hline $\mathrm{C} 4 \mathrm{~B}$ & $0.104(4)$ & $0.047(3)$ & $0.090(4)$ & $0.013(3)$ & $0.026(3)$ & $-0.008(3)$ \\
\hline $\mathrm{C} 5 \mathrm{~A}$ & $0.074(3)$ & $0.062(3)$ & $0.062(3)$ & $-0.003(2)$ & $-0.013(3)$ & $0.002(3)$ \\
\hline C5B & $0.107(4)$ & $0.057(3)$ & $0.069(3)$ & $-0.010(3)$ & $0.026(3)$ & $-0.009(3)$ \\
\hline $\mathrm{C} 6 \mathrm{~A}$ & $0.048(3)$ & $0.047(3)$ & $0.038(3)$ & $0.004(2)$ & $-0.005(2)$ & $0.004(2)$ \\
\hline $\mathrm{C} 6 \mathrm{~B}$ & $0.063(3)$ & $0.043(3)$ & $0.042(3)$ & $0.000(2)$ & $0.004(2)$ & $-0.004(2)$ \\
\hline C7A & $0.065(3)$ & $0.042(3)$ & $0.038(3)$ & $-0.008(2)$ & $-0.003(2)$ & $-0.003(2)$ \\
\hline C7B & $0.064(3)$ & $0.047(3)$ & $0.044(3)$ & $-0.007(3)$ & $-0.002(2)$ & $0.015(2)$ \\
\hline $\mathrm{C} 8 \mathrm{~A}$ & $0.091(11)$ & $0.055(7)$ & $0.056(9)$ & $-0.006(6)$ & $0.006(9)$ & $0.018(9)$ \\
\hline C8B & $0.116(15)$ & $0.062(11)$ & $0.055(9)$ & $0.005(7)$ & $0.038(9)$ & $0.038(8)$ \\
\hline C9A & $0.122(14)$ & $0.058(9)$ & $0.042(7)$ & $-0.009(6)$ & $-0.035(9)$ & $-0.030(8)$ \\
\hline $\mathrm{C} 9 \mathrm{~B}$ & $0.083(9)$ & $0.093(10)$ & $0.057(8)$ & $0.011(7)$ & $0.033(6)$ & $0.019(8)$ \\
\hline
\end{tabular}




$\begin{array}{lcccccc}\text { C10A } & 0.081(13) & 0.158(16) & 0.023(10) & -0.014(10) & -0.019(8) & 0.026(12) \\ \text { C10B } & 0.101(11) & 0.052(8) & 0.096(12) & 0.029(8) & 0.057(7) & 0.000(7) \\ \text { C9C } & 0.112(17) & 0.056(13) & 0.141(18) & -0.030(11) & -0.097(15) & 0.000(13) \\ \text { C9D } & 0.115(16) & 0.065(13) & 0.072(13) & -0.043(10) & 0.014(11) & -0.024(12) \\ \text { C10C } & 0.21(3) & 0.016(8) & 0.028(13) & 0.007(8) & -0.046(14) & 0.008(13) \\ \text { C10D } & 0.117(18) & 0.095(16) & 0.043(11) & -0.042(10) & 0.049(10) & 0.011(14) \\ \text { C8C } & 0.122(19) & 0.120(19) & 0.027(11) & -0.053(12) & -0.009(11) & -0.023(13) \\ \text { C8D } & 0.021(9) & 0.09(2) & 0.066(16) & -0.054(13) & 0.012(10) & -0.034(11)\end{array}$

The Temperature Factor has the Form of $\operatorname{Exp}(-\mathrm{T})$ Where $\mathrm{T}=8 *(\mathrm{P} i * * 2) * \mathrm{U} *(\operatorname{Sin}($ Theta $) /$ Lambda $) * * 2$ for Isotropic Atoms $\mathrm{T}=2 *(\mathrm{Pi} * 2) * \operatorname{Sumij}(\mathrm{h}(i) * \mathrm{~h}(j) * \mathrm{U}(i, j) * A \operatorname{star}(i) * A s t a r(j))$, for Anisotropic Atoms. Astar(i) are Reciprocal Axial Lengths and h(i) are the Reflection Indices. 


\begin{tabular}{|c|c|c|c|c|c|}
\hline V & -0 & $1.588(3)$ & $\mathrm{C} 4 \mathrm{~B}$ & $-C 5 B$ & $1.372(6)$ \\
\hline V & $-01 A$ & $1.883(2)$ & C5A & $-\mathrm{C} 6 \mathrm{~A}$ & $1.378(6)$ \\
\hline V & $-01 B$ & $1.883(2)$ & C5B & $-\mathrm{C} 6 \mathrm{~B}$ & $1.391(6)$ \\
\hline V & $-\mathrm{N} 1 \mathrm{~A}$ & $2.086(3)$ & $\mathrm{C} 6 \mathrm{~A}$ & $-C 7 A$ & $1.469(5)$ \\
\hline V & $-\mathrm{N} 1 \mathrm{~B}$ & $2.079(3)$ & C6B & $-C 7 B$ & $1.481(5)$ \\
\hline O1A & $-\mathrm{C} 1 \mathrm{~A}$ & $1.315(4)$ & C8A & $-C 9 A$ & $1.48(2)$ \\
\hline O1B & $-\mathrm{C} 1 \mathrm{~B}$ & $1.327(4)$ & C8B & $-C 9 B$ & $1.48(2)$ \\
\hline $\mathrm{O} 2 \mathrm{~A}$ & $-C 7 A$ & $1.342(5)$ & $\mathrm{C} 8 \mathrm{C}$ & $-\mathrm{C} 9 \mathrm{C}$ & $1.48(3)$ \\
\hline $\mathrm{O} 2 \mathrm{~A}$ & $-C 8 A$ & $1.413(18)$ & C8D & $-C 9 D$ & $1.48(3)$ \\
\hline $\mathrm{O} 2 \mathrm{~A}$ & $-\mathrm{C} 8 \mathrm{C}$ & $1.54(2)$ & C9A & $-\mathrm{C} 10 \mathrm{~A}$ & $1.48(2)$ \\
\hline $\mathrm{O} 2 \mathrm{~B}$ & $-\mathrm{C} 7 \mathrm{~B}$ & $1.332(5)$ & C9B & $-\mathrm{C} 10 \mathrm{~B}$ & $1.480(19)$ \\
\hline O2B & $-C 8 B$ & $1.36(2)$ & $\mathrm{C} 9 \mathrm{C}$ & $-\mathrm{C} 10 \mathrm{C}$ & $1.48(3)$ \\
\hline $\mathrm{O} 2 \mathrm{~B}$ & $-C 8 D$ & $1.55(3)$ & C9D & -C10D & $1.48(3)$ \\
\hline N1A & $-C 7 A$ & $1.281(5)$ & $\mathrm{C} 2 \mathrm{~A}$ & $-\mathrm{H} 2 \mathrm{AA}$ & 0.9307 \\
\hline N1A & $-\mathrm{C} 10 \mathrm{~A}$ & $1.55(2)$ & $\mathrm{C} 2 \mathrm{~B}$ & $-\mathrm{H} 2 \mathrm{BA}$ & 0.9306 \\
\hline N1A & $-\mathrm{C} 10 \mathrm{C}$ & $1.40(3)$ & C3A & $-\mathrm{H} 3 \mathrm{AA}$ & 0.9295 \\
\hline N1B & $-C 7 B$ & $1.273(6)$ & C3B & $-\mathrm{H} 3 \mathrm{BA}$ & 0.9286 \\
\hline N1B & $-\mathrm{C} 10 \mathrm{~B}$ & $1.45(2)$ & $\mathrm{C} 4 \mathrm{~A}$ & $-\mathrm{H} 4 \mathrm{AA}$ & 0.9295 \\
\hline N1B & $-\mathrm{C} 10 \mathrm{D}$ & $1.55(2)$ & C4B & $-\mathrm{H} 4 \mathrm{BA}$ & 0.9297 \\
\hline $\mathrm{C} 1 \mathrm{~A}$ & $-\mathrm{C} 2 \mathrm{~A}$ & $1.400(5)$ & C5A & $-\mathrm{H} 5 \mathrm{AA}$ & 0.9296 \\
\hline $\mathrm{C} 1 \mathrm{~A}$ & $-\mathrm{C} 6 \mathrm{~A}$ & $1.398(6)$ & C5B & $-\mathrm{H} 5 \mathrm{BA}$ & 0.9292 \\
\hline C1B & $-\mathrm{C} 2 \mathrm{~B}$ & $1.402(5)$ & C8A & $-\mathrm{H} 8 \mathrm{AA}$ & 0.9701 \\
\hline $\mathrm{C} 1 \mathrm{~B}$ & $-\mathrm{C} 6 \mathrm{~B}$ & $1.395(5)$ & C8A & $-\mathrm{H} 8 \mathrm{AB}$ & 0.9699 \\
\hline $\mathrm{C} 2 \mathrm{~A}$ & $-C 3 A$ & $1.365(6)$ & C8B & $-\mathrm{H} 8 \mathrm{BA}$ & 0.9714 \\
\hline C2B & $-C 3 B$ & $1.373(6)$ & C8B & $-\mathrm{H} 8 \mathrm{BB}$ & 0.9695 \\
\hline C3A & $-\mathrm{C} 4 \mathrm{~A}$ & $1.363(7)$ & C8C & $-\mathrm{H} 8 \mathrm{CA}$ & 0.9700 \\
\hline C3E & 4B & $1.370(7)$ & C8C & $-\mathrm{H} 8 \mathrm{CH}$ & 0.96 \\
\hline
\end{tabular}




\begin{tabular}{|c|c|c|c|c|c|}
\hline $\mathrm{C} 4 \mathrm{~A}$ & $-\mathrm{C} 5 \mathrm{~A}$ & $1.372(6)$ & C8D & $-\mathrm{H} 8 \mathrm{DA}$ & 0.9708 \\
\hline C8D & $-\mathrm{H} 8 \mathrm{DB}$ & 0.9687 & $\mathrm{C} 10 \mathrm{~A}$ & $-\mathrm{H} 10 \mathrm{~B}$ & 0.9698 \\
\hline C9A & $-\mathrm{H} 9 \mathrm{AA}$ & 0.9701 & $\mathrm{C} 10 \mathrm{~A}$ & $-\mathrm{H} 10 \mathrm{C}$ & 0.9701 \\
\hline $\mathrm{C} 9 \mathrm{~A}$ & $-\mathrm{H} 9 \mathrm{AB}$ & 0.9693 & $\mathrm{C} 10 \mathrm{~B}$ & $-\mathrm{H} 10 \mathrm{~F}$ & 0.9728 \\
\hline $\mathrm{C} 9 \mathrm{~B}$ & $-\mathrm{H} 9 \mathrm{BA}$ & 0.9705 & $\mathrm{C} 10 \mathrm{~B}$ & $-\mathrm{H} 10 \mathrm{G}$ & 0.9673 \\
\hline C9B & $-\mathrm{H} 9 \mathrm{BB}$ & 0.9690 & $\mathrm{C} 10 \mathrm{C}$ & $-\mathrm{H} 10 \mathrm{~A}$ & 0.9681 \\
\hline $\mathrm{C} 9 \mathrm{C}$ & $-\mathrm{H} 9 \mathrm{CA}$ & 0.9701 & $\mathrm{C} 10 \mathrm{C}$ & $-\mathrm{H} 10 \mathrm{H}$ & 0.9711 \\
\hline $\mathrm{C} 9 \mathrm{C}$ & $-\mathrm{H} 9 \mathrm{CB}$ & 0.9722 & C10D & $-\mathrm{H} 10 \mathrm{D}$ & 0.9744 \\
\hline C9D & $-H 9 D A$ & 0.9731 & $\mathrm{C} 10 \mathrm{D}$ & $-\mathrm{H} 10 \mathrm{E}$ & 0.9656 \\
\hline C9D & $-\mathrm{H} 9 \mathrm{DB}$ & 0.9675 & & & \\
\hline
\end{tabular}




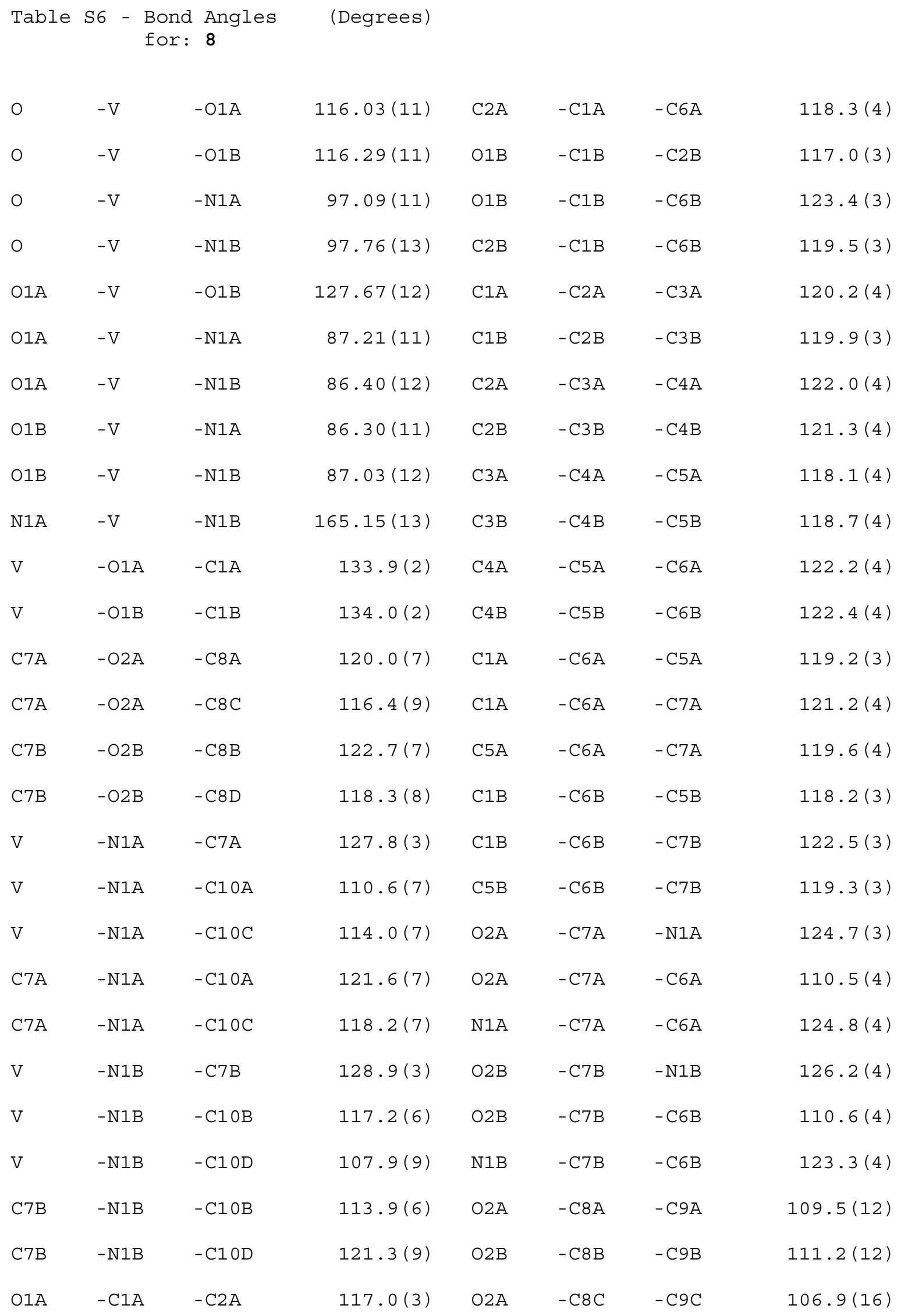




\begin{tabular}{|c|c|c|c|c|c|c|c|}
\hline O1A & $-\mathrm{C} 1 \mathrm{~A}$ & $-\mathrm{C} 6 \mathrm{~A}$ & $124.7(3)$ & $\mathrm{O} 2 \mathrm{~B}$ & $-C 8 D$ & $-C 9 D$ & $111.1(15)$ \\
\hline $\mathrm{C} 8 \mathrm{~A}$ & $-\mathrm{C} 9 \mathrm{~A}$ & $-\mathrm{C} 10 \mathrm{~A}$ & $116.0(14)$ & H8AA & $-C 8 A$ & $-\mathrm{H} 8 \mathrm{AB}$ & 108.25 \\
\hline $\mathrm{C} 8 \mathrm{~B}$ & $-C 9 B$ & $-\mathrm{C} 10 \mathrm{~B}$ & $108.0(11)$ & $\mathrm{O} 2 \mathrm{~B}$ & $-\mathrm{C} 8 \mathrm{~B}$ & $-\mathrm{H} 8 \mathrm{BA}$ & 109.40 \\
\hline $\mathrm{C} 8 \mathrm{C}$ & $-\mathrm{C} 9 \mathrm{C}$ & $-\mathrm{C} 10 \mathrm{C}$ & $106.1(18)$ & $\mathrm{O} 2 \mathrm{~B}$ & $-C 8 B$ & $-\mathrm{H} 8 \mathrm{BB}$ & 109.51 \\
\hline C8D & $-C 9 D$ & $-\mathrm{C} 10 \mathrm{D}$ & $115.2(17)$ & $\mathrm{C} 9 \mathrm{~B}$ & $-C 8 B$ & $-\mathrm{H} 8 \mathrm{BA}$ & 109.29 \\
\hline $\mathrm{N} 1 \mathrm{~A}$ & $-\mathrm{C} 10 \mathrm{~A}$ & $-\mathrm{C} 9 \mathrm{~A}$ & $106.7(13)$ & $\mathrm{C} 9 \mathrm{~B}$ & $-\mathrm{C} 8 \mathrm{~B}$ & $-\mathrm{H} 8 \mathrm{BB}$ & 109.34 \\
\hline $\mathrm{N} 1 \mathrm{~B}$ & $-\mathrm{C} 10 \mathrm{~B}$ & $-C 9 B$ & $112.4(12)$ & H8BA & $-C 8 B$ & $-\mathrm{H} 8 \mathrm{BB}$ & 108.01 \\
\hline $\mathrm{N} 1 \mathrm{~A}$ & $-\mathrm{C} 10 \mathrm{C}$ & $-\mathrm{C} 9 \mathrm{C}$ & $119.1(16)$ & $\mathrm{C} 9 \mathrm{C}$ & $-\mathrm{C} 8 \mathrm{C}$ & $-\mathrm{H} 8 \mathrm{CA}$ & 110.11 \\
\hline $\mathrm{N} 1 \mathrm{~B}$ & $-\mathrm{C} 10 \mathrm{D}$ & $-C 9 D$ & $107.0(18)$ & $\mathrm{C} 9 \mathrm{C}$ & $-\mathrm{C} 8 \mathrm{C}$ & $-\mathrm{H} 8 \mathrm{CB}$ & 110.32 \\
\hline $\mathrm{C} 1 \mathrm{~A}$ & $-\mathrm{C} 2 \mathrm{~A}$ & $-\mathrm{H} 2 \mathrm{AA}$ & 119.92 & $\mathrm{H} 8 \mathrm{CA}$ & $-\mathrm{C} 8 \mathrm{C}$ & $-\mathrm{H} 8 \mathrm{CB}$ & 108.67 \\
\hline $\mathrm{C} 3 \mathrm{~A}$ & $-\mathrm{C} 2 \mathrm{~A}$ & $-\mathrm{H} 2 \mathrm{AA}$ & 119.93 & $\mathrm{O} 2 \mathrm{~A}$ & $-\mathrm{C} 8 \mathrm{C}$ & $-\mathrm{H} 8 \mathrm{CB}$ & 110.46 \\
\hline $\mathrm{C} 1 \mathrm{~B}$ & $-\mathrm{C} 2 \mathrm{~B}$ & $-\mathrm{H} 2 \mathrm{BA}$ & 120.11 & $\mathrm{O} 2 \mathrm{~A}$ & $-\mathrm{C} 8 \mathrm{C}$ & $-\mathrm{H} 8 \mathrm{CA}$ & 110.33 \\
\hline $\mathrm{C} 3 \mathrm{~B}$ & $-C 2 B$ & $-\mathrm{H} 2 \mathrm{BA}$ & 120.01 & $\mathrm{O} 2 \mathrm{~B}$ & $-C 8 D$ & $-\mathrm{H} 8 \mathrm{DB}$ & 109.48 \\
\hline $\mathrm{C} 2 \mathrm{~A}$ & $-C 3 A$ & $-\mathrm{H} 3 \mathrm{AA}$ & 118.97 & $\mathrm{O} 2 \mathrm{~B}$ & $-C 8 D$ & $-\mathrm{H} 8 \mathrm{DA}$ & 109.25 \\
\hline $\mathrm{C} 4 \mathrm{~A}$ & $-C 3 A$ & $-\mathrm{H} 3 \mathrm{AA}$ & 118.99 & $\mathrm{H} 8 \mathrm{DA}$ & $-C 8 D$ & $-\mathrm{H} 8 \mathrm{DB}$ & 108.02 \\
\hline $\mathrm{C} 2 \mathrm{~B}$ & $-C 3 B$ & $-\mathrm{H} 3 \mathrm{BA}$ & 119.32 & C9D & $-C 8 D$ & - H8DA & 109.31 \\
\hline $\mathrm{C} 4 \mathrm{~B}$ & $-C 3 B$ & $-\mathrm{H} 3 \mathrm{BA}$ & 119.39 & C9D & $-C 8 D$ & $-\mathrm{H} 8 \mathrm{DB}$ & 109.62 \\
\hline C3A & $-C 4 A$ & $-\mathrm{H} 4 \mathrm{AA}$ & 121.00 & C8A & $-C 9 A$ & - H9AB & 108.29 \\
\hline C5A & $-\mathrm{C} 4 \mathrm{~A}$ & $-\mathrm{H} 4 \mathrm{AA}$ & 120.93 & H9AA & $-C 9 A$ & - H9AB & 107.37 \\
\hline $\mathrm{C} 3 \mathrm{~B}$ & $-C 4 B$ & $-\mathrm{H} 4 \mathrm{BA}$ & 120.68 & $\mathrm{C} 10 \mathrm{~A}$ & $-C 9 A$ & -H9AA & 108.31 \\
\hline C5B & $-C 4 B$ & $-\mathrm{H} 4 \mathrm{BA}$ & 120.61 & $\mathrm{C} 10 \mathrm{~A}$ & $-C 9 A$ & - H9AB & 108.30 \\
\hline $\mathrm{C} 4 \mathrm{~A}$ & $-\mathrm{C} 5 \mathrm{~A}$ & - H5AА & 118.97 & C8A & $-\mathrm{C} 9 \mathrm{~A}$ & - H9AA & 108.28 \\
\hline $\mathrm{C} 6 \mathrm{~A}$ & $-\mathrm{C} 5 \mathrm{~A}$ & -H5AA & 118.81 & $\mathrm{C} 10 \mathrm{~B}$ & $-C 9 B$ & - H9BA & 109.94 \\
\hline $\mathrm{C} 4 \mathrm{~B}$ & $-C 5 B$ & $-\mathrm{H} 5 \mathrm{BA}$ & 118.80 & $\mathrm{C} 10 \mathrm{~B}$ & $-C 9 B$ & - H9BB & 110.15 \\
\hline $\mathrm{C} 6 \mathrm{~B}$ & $-C 5 B$ & $-\mathrm{H} 5 \mathrm{BA}$ & 118.84 & H9BA & $-C 9 B$ & -Н9BB & 108.48 \\
\hline $\mathrm{O} 2 \mathrm{~A}$ & $-C 8 A$ & $-\mathrm{H} 8 \mathrm{AA}$ & 109.77 & $\mathrm{C} 8 \mathrm{~B}$ & $-C 9 B$ & -Н9BB & 110.18 \\
\hline $\mathrm{O} 2 \mathrm{~A}$ & $-C 8 A$ & $-\mathrm{H} 8 \mathrm{AB}$ & 109.76 & C8B & $-C 9 B$ & -H9BA & 110.11 \\
\hline C9A & $-C 8 A$ & $-\mathrm{H} 8 \mathrm{AA}$ & 109.74 & $\mathrm{H} 9 \mathrm{CA}$ & $-\mathrm{C} 9 \mathrm{C}$ & $-\mathrm{H} 9 \mathrm{CB}$ & 108.54 \\
\hline
\end{tabular}


63

\begin{tabular}{|c|c|c|c|c|c|c|c|}
\hline C9A & $-C 8 A$ & $-\mathrm{H} 8 \mathrm{AB}$ & 109.84 & $\mathrm{C} 10 \mathrm{C}$ & $-\mathrm{C} 9 \mathrm{C}$ & $-\mathrm{H} 9 \mathrm{CB}$ & 110.43 \\
\hline $\mathrm{C} 8 \mathrm{C}$ & $-\mathrm{C} 9 \mathrm{C}$ & $-\mathrm{H} 9 \mathrm{CA}$ & 110.74 & C9B & $-\mathrm{C} 10 \mathrm{~B}$ & $-\mathrm{H} 10 \mathrm{G}$ & 109.35 \\
\hline $\mathrm{C} 8 \mathrm{C}$ & $-\mathrm{C} 9 \mathrm{C}$ & $-\mathrm{H} 9 \mathrm{CB}$ & 110.52 & N1B & $-\mathrm{C} 10 \mathrm{~B}$ & $-\mathrm{H} 10 \mathrm{~F}$ & 108.88 \\
\hline $\mathrm{C} 10 \mathrm{C}$ & $-\mathrm{C} 9 \mathrm{C}$ & $-\mathrm{H} 9 \mathrm{CA}$ & 110.56 & N1B & $-\mathrm{C} 10 \mathrm{~B}$ & $-\mathrm{H} 10 \mathrm{G}$ & 109.26 \\
\hline H9DA & $-C 9 D$ & $-\mathrm{H} 9 \mathrm{DB}$ & 107.46 & C9B & $-\mathrm{C} 10 \mathrm{~B}$ & $-\mathrm{H} 10 \mathrm{~F}$ & 109.01 \\
\hline C8D & $-C 9 D$ & -H9DB & 108.82 & $\mathrm{~N} 1 \mathrm{~A}$ & $-\mathrm{C} 10 \mathrm{C}$ & $-\mathrm{H} 10 \mathrm{~A}$ & 107.66 \\
\hline C10D & $-C 9 D$ & -H9DA & 108.32 & $\mathrm{~N} 1 \mathrm{~A}$ & $-\mathrm{C} 10 \mathrm{C}$ & $-\mathrm{H} 10 \mathrm{H}$ & 107.52 \\
\hline C8D & $-C 9 D$ & -H9DA & 108.38 & $\mathrm{C} 9 \mathrm{C}$ & $-\mathrm{C} 10 \mathrm{C}$ & $-\mathrm{H} 10 \mathrm{~A}$ & 107.49 \\
\hline C10D & $-C 9 D$ & -H9DB & 108.38 & $\mathrm{C} 9 \mathrm{C}$ & $-\mathrm{C} 10 \mathrm{C}$ & $-\mathrm{HIOH}$ & 107.51 \\
\hline $\mathrm{N} 1 \mathrm{~A}$ & $-\mathrm{C} 10 \mathrm{~A}$ & $-\mathrm{H} 10 \mathrm{C}$ & 110.41 & $\mathrm{H} 10 \mathrm{~A}$ & $-\mathrm{C} 10 \mathrm{C}$ & $-\mathrm{H} 10 \mathrm{H}$ & 107.01 \\
\hline C9A & $-\mathrm{C} 10 \mathrm{~A}$ & $-\mathrm{H} 10 \mathrm{~B}$ & 110.40 & $\mathrm{~N} 1 \mathrm{~B}$ & $-C 10 D$ & $-\mathrm{H} 10 \mathrm{D}$ & 110.13 \\
\hline $\mathrm{N} 1 \mathrm{~A}$ & $-\mathrm{C} 10 \mathrm{~A}$ & $-\mathrm{H} 10 \mathrm{~B}$ & 110.38 & $\mathrm{~N} 1 \mathrm{~B}$ & $-\mathrm{C} 10 \mathrm{D}$ & $-\mathrm{H} 10 \mathrm{E}$ & 110.72 \\
\hline $\mathrm{H} 10 \mathrm{~B}$ & $-\mathrm{C} 10 \mathrm{~A}$ & $-\mathrm{H} 10 \mathrm{C}$ & 108.54 & C9D & $-C 10 D$ & $-\mathrm{H} 10 \mathrm{D}$ & 110.04 \\
\hline $\mathrm{C} 9 \mathrm{~A}$ & $-\mathrm{C} 10 \mathrm{~A}$ & $-\mathrm{H} 10 \mathrm{C}$ & 110.36 & C9D & $-C 10 D$ & $-\mathrm{H} 10 \mathrm{E}$ & 110.41 \\
\hline $\mathrm{H} 10 \mathrm{~F}$ & $-\mathrm{C} 10 \mathrm{~B}$ & $-\mathrm{H} 10 \mathrm{G}$ & 107.83 & H1OD & $-C 10 D$ & $-\mathrm{H} 10 \mathrm{E}$ & 108.58 \\
\hline
\end{tabular}


Table S7 - Torsion Angles (Degrees)
for: 8

\begin{tabular}{|c|c|c|c|c|}
\hline 0 & $-\mathrm{V}$ & $-01 \mathrm{~A}$ & $-\mathrm{C} 1 \mathrm{~A}$ & $104.0(3)$ \\
\hline O1B & $-\mathrm{V}$ & $-01 A$ & $-\mathrm{C} 1 \mathrm{~A}$ & $-75.6(3)$ \\
\hline $\mathrm{N} 1 \mathrm{~A}$ & $-\mathrm{V}$ & $-01 A$ & $-\mathrm{C} 1 \mathrm{~A}$ & $7.5(3)$ \\
\hline $\mathrm{N} 1 \mathrm{~B}$ & $-\mathrm{V}$ & $-01 A$ & $-\mathrm{C} 1 \mathrm{~A}$ & $-159.1(3)$ \\
\hline 0 & $-\mathrm{V}$ & $-O 1 B$ & $-C 1 B$ & $87.2(3)$ \\
\hline $\mathrm{O} 1 \mathrm{~A}$ & $-\mathrm{V}$ & $-01 B$ & $-C 1 B$ & $-93.1(3)$ \\
\hline $\mathrm{N} 1 \mathrm{~A}$ & $-\mathrm{V}$ & $-01 B$ & $-C 1 B$ & $-176.7(3)$ \\
\hline $\mathrm{N} 1 \mathrm{~B}$ & $-\mathrm{V}$ & $-01 B$ & $-C 1 B$ & $-10.0(3)$ \\
\hline $\mathrm{O}$ & $-\mathrm{V}$ & $-\mathrm{N} 1 \mathrm{~A}$ & $-C 7 A$ & $-120.7(3)$ \\
\hline $\mathrm{O}$ & $-\mathrm{V}$ & $-\mathrm{N} 1 \mathrm{~A}$ & $-\mathrm{C} 10 \mathrm{~A}$ & $58.3(7)$ \\
\hline $\mathrm{O} 1 \mathrm{~A}$ & $-\mathrm{V}$ & $-N 1 A$ & $-C 7 A$ & $-4.8(3)$ \\
\hline O1A & $-\mathrm{V}$ & $-\mathrm{N} 1 \mathrm{~A}$ & $-\mathrm{C} 10 \mathrm{~A}$ & $174.2(7)$ \\
\hline O1B & $-\mathrm{V}$ & $-\mathrm{N} 1 \mathrm{~A}$ & $-C 7 A$ & $123.2(3)$ \\
\hline O1B & $-\mathrm{V}$ & $-\mathrm{N} 1 \mathrm{~A}$ & $-\mathrm{C10A}$ & $-57.8(7)$ \\
\hline 0 & $-\mathrm{V}$ & $-N 1 B$ & $-C 7 B$ & $-107.8(4)$ \\
\hline 0 & $-\mathrm{V}$ & $-N 1 B$ & $-\mathrm{C} 10 \mathrm{~B}$ & $73.3(8)$ \\
\hline O1A & $-\mathrm{V}$ & $-N 1 B$ & $-C 7 B$ & $136.4(4)$ \\
\hline O1A & $-\mathrm{V}$ & $-N 1 B$ & $-\mathrm{C} 10 \mathrm{~B}$ & $-42.5(8)$ \\
\hline O1B & $-\mathrm{V}$ & $-\mathrm{N} 1 \mathrm{~B}$ & $-C 7 B$ & $8.3(4)$ \\
\hline $\mathrm{O} 1 \mathrm{~B}$ & $-\mathrm{V}$ & $-N 1 B$ & $-\mathrm{C} 10 \mathrm{~B}$ & $-170.6(8)$ \\
\hline $\mathrm{V}$ & $-01 \mathrm{~A}$ & $-\mathrm{C} 1 \mathrm{~A}$ & $-\mathrm{C} 2 \mathrm{~A}$ & $174.5(3)$ \\
\hline $\mathrm{V}$ & $-01 A$ & $-\mathrm{C} 1 \mathrm{~A}$ & $-\mathrm{C} 6 \mathrm{~A}$ & $-5.5(6)$ \\
\hline $\mathrm{V}$ & $-01 B$ & $-C 1 B$ & $-\mathrm{C} 2 \mathrm{~B}$ & $-171.1(2)$ \\
\hline $\mathrm{V}$ & $-01 B$ & $-C 1 B$ & $-\mathrm{C} 6 \mathrm{~B}$ & $7.8(5)$ \\
\hline C8A & $-02 \mathrm{~A}$ & $-C 7 A$ & $-\mathrm{N} 1 \mathrm{~A}$ & $-7.8(9)$ \\
\hline $\mathrm{C} 8 \mathrm{~A}$ & $-02 \mathrm{~A}$ & $-C 7 A$ & $-\mathrm{C} 6 \mathrm{~A}$ & $169.6(8)$ \\
\hline $\mathrm{C} 7$ & $-02 \mathrm{~A}$ & $-C 8 A$ & $-C 9 A$ & $34.1(15)$ \\
\hline
\end{tabular}




\begin{tabular}{|c|c|c|c|c|}
\hline $\mathrm{C} 8 \mathrm{~B}$ & $-02 \mathrm{~B}$ & $-C 7 B$ & $-N 1 B$ & $-2.4(11)$ \\
\hline C8B & $-02 B$ & $-C 7 B$ & $-\mathrm{C} 6 \mathrm{~B}$ & $177.3(9)$ \\
\hline $\mathrm{C} 7 \mathrm{~B}$ & $-02 \mathrm{~B}$ & $-C 8 B$ & $-C 9 B$ & $-20.0(16)$ \\
\hline V & $-\mathrm{N} 1 \mathrm{~A}$ & $-\mathrm{C} 10 \mathrm{~A}$ & $-C 9 A$ & $164.0(9)$ \\
\hline C7A & $-\mathrm{N} 1 \mathrm{~A}$ & $-\mathrm{C} 10 \mathrm{~A}$ & $-C 9 A$ & $-16.9(15)$ \\
\hline V & $-\mathrm{N} 1 \mathrm{~A}$ & $-C 7 A$ & $-C 6 A$ & $0.5(5)$ \\
\hline $\mathrm{C} 10 \mathrm{~A}$ & $-\mathrm{N} 1 \mathrm{~A}$ & $-C 7 A$ & $-02 \mathrm{~A}$ & $-1.4(9)$ \\
\hline V & $-\mathrm{N} 1 \mathrm{~A}$ & $-C 7 A$ & $-02 \mathrm{~A}$ & $177.5(2)$ \\
\hline C10A & $-\mathrm{N} 1 \mathrm{~A}$ & $-C 7 A$ & $-\mathrm{C} 6 \mathrm{~A}$ & $-178.5(8)$ \\
\hline V & $-\mathrm{N} 1 \mathrm{~B}$ & $-C 7 B$ & $-02 \mathrm{~B}$ & $174.8(3)$ \\
\hline V & $-N 1 B$ & $-C 7 B$ & $-\mathrm{C} 6 \mathrm{~B}$ & $-4.8(6)$ \\
\hline $\mathrm{C} 10 \mathrm{~B}$ & $-\mathrm{N} 1 \mathrm{~B}$ & $-C 7 B$ & $-02 B$ & $-6.3(10)$ \\
\hline $\mathrm{C} 10 \mathrm{~B}$ & $-\mathrm{N} 1 \mathrm{~B}$ & $-C 7 B$ & $-\mathrm{C} 6 \mathrm{~B}$ & $174.1(8)$ \\
\hline V & $-\mathrm{N} 1 \mathrm{~B}$ & $-\mathrm{C} 10 \mathrm{~B}$ & $-\mathrm{C} 9 \mathrm{~B}$ & $-144.0(9)$ \\
\hline C7B & $-\mathrm{N} 1 \mathrm{~B}$ & $-\mathrm{C} 10 \mathrm{~B}$ & $-\mathrm{C} 9 \mathrm{~B}$ & $37.0(14)$ \\
\hline C6A & $-\mathrm{C} 1 \mathrm{~A}$ & $-\mathrm{C} 2 \mathrm{~A}$ & $-C 3 A$ & $1.3(6)$ \\
\hline $\mathrm{O} 1 \mathrm{~A}$ & $-\mathrm{C} 1 \mathrm{~A}$ & $-\mathrm{C} 6 \mathrm{~A}$ & $-\mathrm{C} 5 \mathrm{~A}$ & $179.4(3)$ \\
\hline O1A & $-\mathrm{C} 1 \mathrm{~A}$ & $-\mathrm{C} 2 \mathrm{~A}$ & $-C 3 A$ & $-178.7(4)$ \\
\hline $\mathrm{C} 2 \mathrm{~A}$ & $-\mathrm{C} 1 \mathrm{~A}$ & $-\mathrm{C} 6 \mathrm{~A}$ & $-C 7 A$ & $177.9(3)$ \\
\hline O1A & $-\mathrm{C} 1 \mathrm{~A}$ & $-\mathrm{C} 6 \mathrm{~A}$ & $-C 7 A$ & $-2.1(6)$ \\
\hline $\mathrm{C} 2 \mathrm{~A}$ & $-\mathrm{C} 1 \mathrm{~A}$ & $-\mathrm{C} 6 \mathrm{~A}$ & $-\mathrm{C} 5 \mathrm{~A}$ & $-0.5(5)$ \\
\hline $\mathrm{O} 1 \mathrm{~B}$ & $-\mathrm{C} 1 \mathrm{~B}$ & $-\mathrm{C} 2 \mathrm{~B}$ & $-C 3 B$ & $178.8(3)$ \\
\hline $\mathrm{C} 6 \mathrm{~B}$ & $-\mathrm{C} 1 \mathrm{~B}$ & $-C 2 B$ & $-C 3 B$ & $-0.1(5)$ \\
\hline O1B & $-\mathrm{C} 1 \mathrm{~B}$ & $-\mathrm{C} 6 \mathrm{~B}$ & $-\mathrm{C} 5 \mathrm{~B}$ & $179.9(4)$ \\
\hline O1B & $-\mathrm{C} 1 \mathrm{~B}$ & $-\mathrm{C} 6 \mathrm{~B}$ & $-C 7 B$ & $0.3(6)$ \\
\hline $\mathrm{C} 2 \mathrm{~B}$ & $-\mathrm{C} 1 \mathrm{~B}$ & $-\mathrm{C} 6 \mathrm{~B}$ & $-\mathrm{C} 5 \mathrm{~B}$ & $-1.3(5)$ \\
\hline $\mathrm{C} 2 \mathrm{~B}$ & $-C 1 B$ & $-\mathrm{C} 6 \mathrm{~B}$ & $-C 7 B$ & $179.1(3)$ \\
\hline$C 1 A$ & $-\mathrm{C} 2 \mathrm{~A}$ & $-C 3 A$ & $-\mathrm{C} 4 \mathrm{~A}$ & $-1.9(6)$ \\
\hline
\end{tabular}




\begin{tabular}{|c|c|c|c|c|}
\hline $\mathrm{C} 1 \mathrm{~B}$ & $-\mathrm{C} 2 \mathrm{~B}$ & $-C 3 B$ & $-C 4 B$ & $2.3(6)$ \\
\hline $\mathrm{C} 2 \mathrm{~A}$ & $-C 3 A$ & $-C 4 A$ & $-\mathrm{C} 5 \mathrm{~A}$ & $1.7(7)$ \\
\hline $\mathrm{C} 2 \mathrm{~B}$ & $-C 3 B$ & $-C 4 B$ & $-C 5 B$ & $-2.9(6)$ \\
\hline C3A & $-\mathrm{C} 4 \mathrm{~A}$ & $-\mathrm{C} 5 \mathrm{~A}$ & $-\mathrm{C} 6 \mathrm{~A}$ & $-0.9(6)$ \\
\hline $\mathrm{C} 3 \mathrm{~B}$ & $-C 4 B$ & $-C 5 B$ & $-\mathrm{C} 6 \mathrm{~B}$ & $1.4(7)$ \\
\hline $\mathrm{C} 4 \mathrm{~A}$ & $-\mathrm{C} 5 \mathrm{~A}$ & $-\mathrm{C} 6 \mathrm{~A}$ & $-C 1 A$ & $0.4(6)$ \\
\hline $\mathrm{C} 4 \mathrm{~A}$ & $-\mathrm{C} 5 \mathrm{~A}$ & $-C 6 A$ & $-C 7 A$ & $-178.1(4)$ \\
\hline $\mathrm{C} 4 \mathrm{~B}$ & $-C 5 B$ & $-\mathrm{C} 6 \mathrm{~B}$ & $-C 1 B$ & $0.7(6)$ \\
\hline $\mathrm{C} 4 \mathrm{~B}$ & $-C 5 B$ & $-C 6 B$ & $-C 7 B$ & $-179.7(4)$ \\
\hline $\mathrm{C} 1 \mathrm{~A}$ & $-\mathrm{C} 6 \mathrm{~A}$ & $-C 7 A$ & $-\mathrm{N} 1 \mathrm{~A}$ & $4.2(6)$ \\
\hline C5A & $-\mathrm{C} 6 \mathrm{~A}$ & $-C 7 A$ & $-02 A$ & $5.2(5)$ \\
\hline C5A & $-\mathrm{C} 6 \mathrm{~A}$ & $-C 7 A$ & $-N 1 A$ & $-177.3(4)$ \\
\hline $\mathrm{C} 1 \mathrm{~A}$ & $-\mathrm{C} 6 \mathrm{~A}$ & $-C 7 A$ & $-02 A$ & $-173.2(3)$ \\
\hline $\mathrm{C} 1 \mathrm{~B}$ & $-\mathrm{C} 6 \mathrm{~B}$ & $-C 7 B$ & $-02 B$ & $179.1(3)$ \\
\hline $\mathrm{C} 1 \mathrm{~B}$ & $-\mathrm{C} 6 \mathrm{~B}$ & $-C 7 B$ & $-N 1 B$ & $-1.2(6)$ \\
\hline $\mathrm{C} 5 \mathrm{~B}$ & $-\mathrm{C} 6 \mathrm{~B}$ & $-C 7 B$ & $-02 B$ & $-0.5(5)$ \\
\hline $\mathrm{C} 5 \mathrm{~B}$ & $-C 6 B$ & $-C 7 B$ & $-N 1 B$ & $179.2(4)$ \\
\hline $\mathrm{O} 2 \mathrm{~A}$ & $-\mathrm{C} 8 \mathrm{~A}$ & $-C 9 A$ & $-\mathrm{C} 10 \mathrm{~A}$ & $-54.5(18)$ \\
\hline $\mathrm{O} 2 \mathrm{~B}$ & $-C 8 B$ & $-C 9 B$ & $-\mathrm{C} 10 \mathrm{~B}$ & $47.6(17)$ \\
\hline $\mathrm{C} 8 \mathrm{~A}$ & $-C 9 A$ & $-\mathrm{C} 10 \mathrm{~A}$ & $-\mathrm{N} 1 \mathrm{~A}$ & $44.7(18)$ \\
\hline $\mathrm{C} 8 \mathrm{~B}$ & $-C 9 B$ & $-\mathrm{C} 10 \mathrm{~B}$ & $-N 1 B$ & $-57.4(16)$ \\
\hline
\end{tabular}


Table s8 - Contact Distances (Angstrom)
for: 8

\begin{tabular}{|c|c|c|c|c|c|}
\hline $\mathrm{V}$ & . C9B_a & $3.953(11)$ & O1B & . H9CB_b & 2.8858 \\
\hline V & . C9C_b & $3.91(2)$ & $\mathrm{O} 2 \mathrm{~A}$ & . H5AA & 2.2632 \\
\hline V & .H9BB_a & 3.4741 & $\mathrm{O} 2 \mathrm{~B}$ & . $\mathrm{H} 5 \mathrm{BA}$ & 2.2680 \\
\hline $\mathrm{V}$ & .H8AA_b & 3.5063 & $\mathrm{~N} 1 \mathrm{~B}$ & .H10D_a & 2.8628 \\
\hline $\mathrm{V}$ & .H9BA_a & 3.4836 & $\mathrm{C} 1 \mathrm{~A}$ & . C9B_a & $3.494(12)$ \\
\hline $\mathrm{V}$ & .H9CA_b & 3.5279 & $\mathrm{C} 1 \mathrm{~B}$ & . C9C_b & $3.563(18)$ \\
\hline O & . C8A_b & $3.081(17)$ & $\mathrm{C} 2 \mathrm{~B}$ & . C3A_d & $3.506(5)$ \\
\hline $\mathrm{O}$ & . $C 8 B \_C$ & $3.292(18)$ & C3A & . $\mathrm{C} 2 \mathrm{~B} \_\mathrm{f}$ & $3.506(5)$ \\
\hline $\mathrm{O}$ & . C9C_b & $3.24(2)$ & C3B & . C7A_e & $3.453(5)$ \\
\hline O & . C8D_C & $3.34(3)$ & $\mathrm{C} 4 \mathrm{~A}$ & .C7A_g & $3.599(6)$ \\
\hline $\mathrm{O}$ & . C9D_C & $3.25(3)$ & C4A & . C6A_g & $3.568(6)$ \\
\hline $\mathrm{O}$ & . $\mathrm{C} 8 \mathrm{C} \_\mathrm{b}$ & $3.19(2)$ & C6A & . $C 4 A_{2} i$ & $3.568(6)$ \\
\hline $\mathrm{O} 1 \mathrm{~A}$ & . C9B_a & $3.300(11)$ & C7A & . C4A_i & $3.599(6)$ \\
\hline $\mathrm{O}$ &. $\mathrm{H} 10 \mathrm{H}$ & 2.6621 & C7A &.$C 3 B_{-} j$ & $3.453(5)$ \\
\hline $\mathrm{O}$ & .H8BB_C & 2.3668 & $\mathrm{C} 7 \mathrm{~B}$ & . C10D_a & $3.48(3)$ \\
\hline $\mathrm{O}$ & H9DB_C & 2.4582 & C8A & $.0 \_k$ & $3.081(17)$ \\
\hline $\mathrm{O}$ & .H9AB_b & 2.8979 & $\mathrm{C} 8 \mathrm{~B}$ & . O_a & $3.292(18)$ \\
\hline $\mathrm{O}$ & .H8AA_b & 2.8669 & $\mathrm{C} 8 \mathrm{C}$ & $.0 \_k$ & $3.19(2)$ \\
\hline $\mathrm{O}$ & .H8AB_b & 2.6138 & C8D & . O_a & $3.34(3)$ \\
\hline $\mathrm{O}$ & . H8CB_b & 2.4552 & $\mathrm{C} 9 \mathrm{~B}$ & $.01 A_{-} \mathrm{C}$ & $3.300(11)$ \\
\hline $\mathrm{O}$ & .H9CA_b & 2.8794 & $\mathrm{C} 9 \mathrm{~B}$ & . C1A_C & $3.494(12)$ \\
\hline $\mathrm{O}$ & .H8DA_C & 2.8004 & $\mathrm{C} 9 \mathrm{~B}$ & $\cdot V_{-} \mathrm{C}$ & $3.953(11)$ \\
\hline $\mathrm{O} 1 \mathrm{~A}$ &. $\mathrm{H} 10 \mathrm{G}$ & 2.6494 & $\mathrm{C} 9 \mathrm{C}$ & $\cdot V_{-} \mathrm{k}$ & $3.91(2)$ \\
\hline $\mathrm{O} 1 \mathrm{~A}$ & . H10E & 2.3985 & $\mathrm{C} 9 \mathrm{C}$ & . O_k & $3.24(2)$ \\
\hline $\mathrm{O} 1 \mathrm{~A}$ &. $\mathrm{H} 10 \mathrm{~F}$ & 2.8659 & $\mathrm{C} 9 \mathrm{C}$ & . $\mathrm{C} 1 \mathrm{~B} \_\mathrm{k}$ & $3.563(18)$ \\
\hline $\mathrm{O} 1 \mathrm{~A}$ & .H9BA_a & 2.5194 & C9D & . O_a & $3.25(3)$ \\
\hline O1B &. $\mathrm{H} 10 \mathrm{C}$ & 2.4461 & C10D & . C7B_C & $3.48(3)$ \\
\hline
\end{tabular}




\begin{tabular}{|c|c|c|c|c|c|}
\hline O1B &. $\mathrm{H} 10 \mathrm{~A}$ & 2.6173 & $\mathrm{C} 1 \mathrm{~A}$ & .H8DB_a & 2.7553 \\
\hline $\mathrm{C} 1 \mathrm{~A}$ & .H9BA_a & 2.7884 & $\mathrm{H} 4 \mathrm{AA}$ & . C7A_9 & 3.0288 \\
\hline $\mathrm{C} 1 \mathrm{~B}$ & . H9CB_b & 2.7698 & H5AA & .H5BA_1 & 2.3636 \\
\hline $\mathrm{C} 2 \mathrm{~A}$ & .H9BA_a & 2.9842 & H5AA & $.02 \mathrm{~A}$ & 2.2632 \\
\hline $\mathrm{C} 2 \mathrm{~A}$ & .H8DB_a & 2.8727 & $\mathrm{H} 8 \mathrm{CA}$ & . $\mathrm{C} 2 \mathrm{~B} \_\mathrm{j}$ & 3.0158 \\
\hline $\mathrm{C} 2 \mathrm{~B}$ & .H8CA_e & 3.0158 & $\mathrm{H} 8 \mathrm{CA}$ & . H10A & 2.4579 \\
\hline C3A & . H2BA_f & 2.9759 & $\mathrm{H} 8 \mathrm{CA}$ & . C3B_j & 2.9331 \\
\hline $\mathrm{C} 3 \mathrm{~B}$ & .H8CA_e & 2.9331 & $\mathrm{H} 8 \mathrm{CB}$ & $.0 \_\mathrm{k}$ & 2.4552 \\
\hline C4B & .H8AB_h & 3.0550 & $\mathrm{H} 9 \mathrm{CA}$ & . V_k & 3.5279 \\
\hline $\mathrm{C} 6 \mathrm{~A}$ & .H3BA_j & 3.0316 & $\mathrm{H} 9 \mathrm{CA}$ & . C7B_k & 3.0989 \\
\hline $\mathrm{C} 6 \mathrm{~A}$ & . H4AA_i & 3.0891 & H9CA & $.0 \_k$ & 2.8794 \\
\hline $\mathrm{C} 6 \mathrm{~B}$ & .H9CB_b & 3.0963 & H9CB & $.01 B \_k$ & 2.8858 \\
\hline C7A & . H9CB & 2.9914 & H9CB &.$C 7 A$ & 2.9914 \\
\hline C7A & . H9AA & 2.9060 & H9CB & . C1B_k & 2.7698 \\
\hline C7A & .H3BA_j & 2.8893 & H9CB & . $\mathrm{C} 6 \mathrm{~B} \_\mathrm{k}$ & 3.0963 \\
\hline C7A & . H4AA_i & 3.0288 & H8AA & . O_k & 2.8669 \\
\hline C7B & . H9BA & 2.8735 & H8AA & .H3AA_i & 2.3248 \\
\hline C7B & .H9CA_b & 3.0989 & H8AA & $\cdot V_{-} k$ & 3.5063 \\
\hline C7B & .H10G_a & 3.0395 & H8AB & . O_k & 2.6138 \\
\hline C7B & .H10D_a & 2.9509 & H8AB & . C4B_l & 3.0550 \\
\hline C7B & . H9DB & 2.9998 & H10A & . $\mathrm{H} 8 \mathrm{CA}$ & 2.4579 \\
\hline C8A & .H3AA_i & 3.0706 & H10A & $.01 B$ & 2.6173 \\
\hline C9A & . H2AA_d & 3.0604 & $\mathrm{H} 10 \mathrm{~A}$ & .H2AA_d & 2.5133 \\
\hline $\mathrm{H} 2 \mathrm{AA}$ & . C9A_f & 3.0604 & $\mathrm{H} 10 \mathrm{C}$ & $.01 B$ & 2.4461 \\
\hline $\mathrm{H} 2 \mathrm{AA}$ &. $\mathrm{H} 10 \mathrm{C} \_f$ & 2.4870 & $\mathrm{H} 10 \mathrm{C}$ & . H2AA_d & 2.4870 \\
\hline $\mathrm{H} 2 \mathrm{AA}$ &. $\mathrm{H} 10 \mathrm{~A} \_\mathrm{f}$ & 2.5133 & H10D & . N1B_C & 2.8628 \\
\hline H3AA & . C8A_9 & 3.0706 & H10D & . C7B_C & 2.9509 \\
\hline H3AA & .H8AA_g & 2.3248 & $\mathrm{H} 10 \mathrm{E}$ & $.01 \mathrm{~A}$ & 2.3985 \\
\hline
\end{tabular}




\begin{tabular}{|c|c|c|c|c|c|}
\hline $\mathrm{H} 4 \mathrm{AA}$ & . C6A_g & 3.0891 & $\mathrm{H} 10 \mathrm{~F}$ & . H8BB & 2.5192 \\
\hline H1 OF & $.01 \mathrm{~A}$ & 2.8659 & $\mathrm{H} 8 \mathrm{DB}$ & . $\mathrm{C} 1 \mathrm{~A} \_\mathrm{C}$ & 2.7553 \\
\hline H10G & $.01 \mathrm{~A}$ & 2.6494 & H8DB & . $\mathrm{C} 2 \mathrm{~A} \_\mathrm{C}$ & 2.8727 \\
\hline H10G & . C7B_C & 3.0395 & H9DB & . O_a & 2.4582 \\
\hline $\mathrm{H} 10 \mathrm{H}$ & .0 & 2.6621 & H9DB &. $\mathrm{C} 7 \mathrm{~B}$ & 2.9998 \\
\hline H9AA &.$C 7 A$ & 2.9060 & H8BB & . O_a & 2.3668 \\
\hline H9AB & $.0 \_\mathrm{k}$ & 2.8979 & H8BB &. $\mathrm{H} 10 \mathrm{~F}$ & 2.5192 \\
\hline $\mathrm{H} 2 \mathrm{BA}$ & . C3A_d & 2.9759 & H9BA & . $\mathrm{C} 2 \mathrm{~A} \_\mathrm{C}$ & 2.9842 \\
\hline H3 BA & . C7A_e & 2.8893 & H9BA &. $\mathrm{C} 7 \mathrm{~B}$ & 2.8735 \\
\hline H3 BA & . C6A_e & 3.0316 & H9BA & . V_C & 3.4836 \\
\hline H5BA & $.02 B$ & 2.2680 & H9BA & $.01 A_{-} \mathrm{C}$ & 2.5194 \\
\hline H5BA & .H5AA_h & 2.3636 & H9BA & . $\mathrm{C} 1 \mathrm{~A}_{-} \mathrm{C}$ & 2.7884 \\
\hline H8DA & . O_a & 2.8004 & H9BB & . V_C & 3.4741 \\
\hline
\end{tabular}




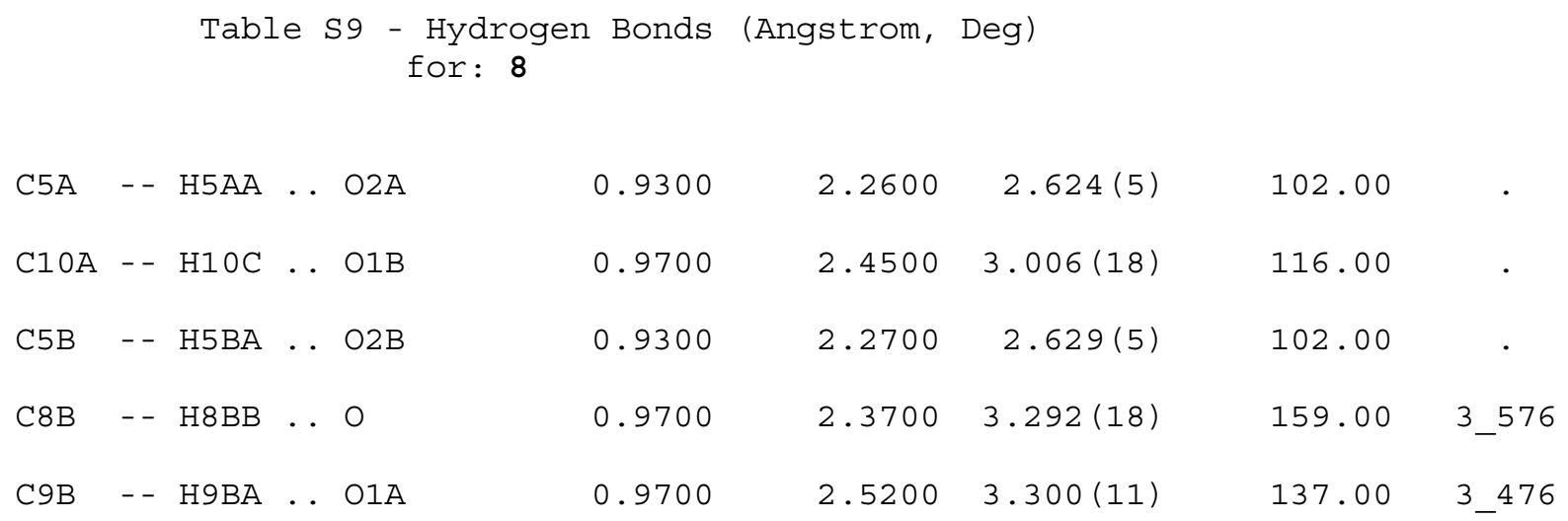


Translation of Symmetry Code to Equiv.Pos

\begin{tabular}{|c|c|c|}
\hline & & 1 \\
\hline & 5.00 & $=-x, 1 / 2+y, 1 / 2-z$ \\
\hline & 3476.00 & $=-1 / 2+x, 5 / 2-y, 1-z$ \\
\hline & \pm .00 & $=1 / 2-x, 2-y,-1 / 2+z$ \\
\hline & & $=1-x$ \\
\hline & 0 & $=1 / 2-x, 2-$ \\
\hline & 3566.00 & $=1 / 2+x, 3 / 2-y, 1-z$ \\
\hline & 1565.00 & $=x, 1+y, z$ \\
\hline & 3466.00 & $=-1 / 2+x, 3 / 2-y, 1-z$ \\
\hline & 4645.00 & $=1-x,-1 / 2+y, 1 / 2-z$ \\
\hline & .00 & $-x,-1 / 2+y, 1 / 2-z$ \\
\hline & 5.00 & $x,-1+y$, \\
\hline
\end{tabular}


Table S1 - Crystal Data and Details of the Structure Determination
for: 9 Crystal Data

Formula

Formula Weight

Crystal system

Space group

$a, b, c$ [Angstrom]

alpha, beta, gamma [deg]

$\mathrm{V} \quad$ [Ang**3]

Z

$\mathrm{D}(\mathrm{calc}) \quad[\mathrm{g} / \mathrm{cm} * \star 3]$

$\mathrm{Mu}(\mathrm{MoKa}) \quad[/ \mathrm{mm}]$

$F(000)$

Crystal size [mm]
C22 H23 Mn N2 O6

466.36

Monoclinic

$\mathrm{P} 21 / \mathrm{C}$

(No. 14)

$14.134(8)$

$9.851(10)$

$15.474(15)$

$90 \quad 107.872(17)$

90

$2051(3)$

4

1.510

0.687

968

$0.00 \times 0.00 \times 0.00$

Data Collection

Temperature ( $\mathrm{K})$

Radiation [Angstrom]

MoKa

0.71073

Theta Min-Max [Deg]

$2.5, \quad 28.4$

Dataset

$-18: 18 ;-12: 11 ;-20: 17$

Tot., Uniq. Data, R(int)

$9132 ，$

4406 ,

0.051

Observed data [I $>2.0$ sigma(I)]

2914

Refinement

Nref, Npar

$4406, \quad 280$

R， WR2， S

$0.0720,0.2185,1.04$

$\mathrm{W}=1 /\left[\backslash \mathrm{S}^{\wedge} 2^{\wedge}\left(\mathrm{FO} \mathrm{O}^{\wedge} 2^{\wedge}\right)+(0.1347 \mathrm{P})^{\wedge} 2^{\wedge}\right]$

where $\mathrm{P}=\left(\mathrm{FO} \mathrm{O}^{\wedge} 2^{\wedge}+2 \mathrm{FC}^{\wedge} 2^{\wedge}\right) / 3$

Max. and Av. Shift/Error

$0.00,0.00$

Min. and Max. Resd. Dens. [e/Ang^3]

$-1.53,1.06$ 
Table S2 - Final Coordinates and Equivalent Isotropic Displacement Parameters of the non-Hydrogen atoms

for: 9

\begin{tabular}{|c|c|c|c|c|}
\hline Atom & $\mathrm{x}$ & Y & $z$ & $\mathrm{U}($ eq $) \quad\left[\mathrm{Ang}^{\wedge} 2\right]$ \\
\hline--- & --- & --- & --- & ---------- \\
\hline Mn & $0.25600(5)$ & $0.62735(5)$ & $0.20844(4)$ & $0.0305(2)$ \\
\hline $\mathrm{O} 1$ & $0.3432(3)$ & $0.5182(4)$ & $0.3368(2)$ & $0.0530(12)$ \\
\hline $\mathrm{O} A \mathrm{~A}$ & $0.2870(2)$ & $0.7997(3)$ & $0.25940(19)$ & $0.0356(9)$ \\
\hline $\mathrm{O} B \mathrm{~B}$ & $0.2252(2)$ & $0.4586(3)$ & $0.1528(2)$ & $0.0356(9)$ \\
\hline $\mathrm{O} 2$ & $0.1879(3)$ & $0.5743(3)$ & $0.3077(2)$ & $0.0526(11)$ \\
\hline $\mathrm{O} 2 \mathrm{~A}$ & $0.0043(3)$ & $0.8737(3)$ & $0.0706(2)$ & $0.0506(11)$ \\
\hline $\mathrm{O} 2 \mathrm{~B}$ & $0.4886(2)$ & $0.5476(3)$ & $0.0922(2)$ & $0.0407(10)$ \\
\hline N1A & $0.1350(3)$ & $0.7179(3)$ & $0.1101(2)$ & $0.0357(11)$ \\
\hline N1B & $0.3635(3)$ & $0.6446(3)$ & $0.1420(2)$ & $0.0327(10)$ \\
\hline $\mathrm{C} 1$ & $0.2638(4)$ & $0.5142(4)$ & $0.3574(3)$ & $0.0430(16)$ \\
\hline $\mathrm{C} 1 \mathrm{~A}$ & $0.2193(3)$ & $0.8823(3)$ & $0.2740(3)$ & $0.0308(11)$ \\
\hline $\mathrm{C} 1 \mathrm{~B}$ & $0.2967(3)$ & $0.3682(3)$ & $0.1580(3)$ & $0.0281(11)$ \\
\hline $\mathrm{C} 2$ & $0.2581(6)$ & $0.4370(5)$ & $0.4382(4)$ & $0.068(2)$ \\
\hline $\mathrm{C} 2 \mathrm{~A}$ & $0.2474(4)$ & $0.9647(4)$ & $0.3517(3)$ & $0.0406(14)$ \\
\hline $\mathrm{C} 2 \mathrm{~B}$ & $0.2794(4)$ & $0.2317(4)$ & $0.1764(3)$ & $0.0351(13)$ \\
\hline C3A & $0.1816(5)$ & $1.0530(4)$ & $0.3709(3)$ & $0.0524(18)$ \\
\hline C3B & $0.3499(4)$ & $0.1323(4)$ & $0.1792(3)$ & $0.0363(13)$ \\
\hline $\mathrm{C} 4 \mathrm{~A}$ & $0.0851(5)$ & $1.0639(5)$ & $0.3133(4)$ & $0.0580(19)$ \\
\hline C4B & $0.4381(4)$ & $0.1633(4)$ & $0.1650(3)$ & $0.0371(13)$ \\
\hline C5A & $0.0556(4)$ & $0.9865(5)$ & $0.2362(3)$ & $0.0477(16)$ \\
\hline C5B & $0.4590(3)$ & $0.2980(4)$ & $0.1495(3)$ & $0.0331(12)$ \\
\hline $\mathrm{C} 6 \mathrm{~A}$ & $0.1204(4)$ & $0.8949(4)$ & $0.2144(3)$ & $0.0337(11)$ \\
\hline C6B & $0.3893(3)$ & $0.4011(3)$ & $0.1456(3)$ & $0.0276(11)$ \\
\hline C7A & $0.0879(3)$ & $0.8205(4)$ & $0.1283(3)$ & $0.0341(13)$ \\
\hline C7B & $0.4123(3)$ & $0.5421(4)$ & $0.1264(2)$ & $0.0290(11)$ \\
\hline
\end{tabular}




$\begin{array}{lcccc}\text { C8A } & -0.0400(4) & 0.8035(5) & -0.0142(3) & 0.0556(17) \\ \text { C8B } & 0.5262(4) & 0.6820(4) & 0.0800(3) & 0.0417(16) \\ \text { C9A } & 0.0357(5) & 0.7499(5) & -0.0519(3) & 0.0613(19) \\ \text { C9B } & 0.4422(4) & 0.7791(4) & 0.0462(3) & 0.0464(16) \\ \text { C10A } & 0.1044(4) & 0.6567(5) & 0.0182(3) & 0.0520(17) \\ \text { C10B } & 0.3853(4) & 0.7829(4) & 0.1150(3) & 0.0425(15)\end{array}$

$\mathrm{U}($ eq) $=1 / 3$ of the trace of the orthogonalized $U$ Tensor 
Table S3 - Hydrogen Atom Positions and Isotropic Displacement Parameters

for : 9

\begin{tabular}{|c|c|c|c|c|c|}
\hline Atom & $\mathrm{x}$ & Y & z & U (iso) & {$\left[\mathrm{Ang}^{\wedge} 2\right]$} \\
\hline---- & --- & --- & --- & ----- & ----- \\
\hline $\mathrm{H} 2 \mathrm{AA}$ & 0.31200 & 0.95930 & 0.39090 & & 0.0490 \\
\hline H3AА & 0.20190 & 1.10610 & 0.42300 & & 0.0630 \\
\hline $\mathrm{H} 2 \mathrm{~A}$ & 0.30730 & 0.47070 & 0.49150 & & 0.1020 \\
\hline $\mathrm{H} 2 \mathrm{~B}$ & 0.27010 & 0.34250 & 0.43050 & & 0.1020 \\
\hline $\mathrm{H} 2 \mathrm{C}$ & 0.19320 & 0.44790 & 0.44490 & & 0.1020 \\
\hline $\mathrm{H} 4 \mathrm{AA}$ & 0.04060 & 1.12340 & 0.32690 & & 0.0690 \\
\hline H5AA & -0.00910 & 0.99500 & 0.19740 & & 0.0570 \\
\hline H8AA & -0.08300 & 0.86550 & -0.05750 & & 0.0670 \\
\hline H8AB & -0.08060 & 0.72930 & -0.00430 & & 0.0670 \\
\hline H9AА & 0.07350 & 0.82400 & -0.06640 & & 0.0730 \\
\hline H9AB & 0.00420 & 0.69980 & -0.10730 & & 0.0730 \\
\hline $\mathrm{H} 8 \mathrm{C}$ & 0.56350 & 0.67670 & 0.03690 & & 0.0500 \\
\hline H8D & 0.57070 & 0.71400 & 0.13750 & & 0.0500 \\
\hline $\mathrm{H} 2 \mathrm{BA}$ & 0.22020 & 0.20810 & 0.18680 & & 0.0420 \\
\hline Н3 ВА & 0.33680 & 0.04290 & 0.19100 & & 0.0430 \\
\hline $\mathrm{H} 10 \mathrm{~A}$ & 0.07070 & 0.57140 & 0.01970 & & 0.0630 \\
\hline $\mathrm{H} 10 \mathrm{~B}$ & 0.16310 & 0.63740 & 0.00040 & & 0.0630 \\
\hline $\mathrm{H} 4 \mathrm{BA}$ & 0.48390 & 0.09520 & 0.16550 & & 0.0450 \\
\hline H5BA & 0.51990 & 0.31970 & 0.14180 & & 0.0400 \\
\hline $\mathrm{H} 10 \mathrm{BA}$ & 0.42400 & 0.83210 & 0.16850 & & 0.0510 \\
\hline $\mathrm{H} 10 \mathrm{BB}$ & 0.32330 & 0.83140 & 0.08910 & & 0.0510 \\
\hline H9BA & 0.39890 & 0.74980 & -0.01240 & & 0.0550 \\
\hline H9BB & 0.46740 & 0.86880 & 0.03940 & & 0.0550 \\
\hline
\end{tabular}

The Temperature Factor has the Form of $\operatorname{Exp}(-\mathrm{T})$ Where $\mathrm{T}=8 *(\mathrm{Pi} * 2) * \mathrm{U} *(\mathrm{Sin}($ Theta $) /$ Lambda $) * * 2$ for Isotropic Atoms 


\begin{tabular}{|c|c|c|c|c|c|c|}
\hline Atom & $\mathrm{U}(1,1)$ or & $\mathrm{U}(2,2)$ & $\mathrm{U}(3,3)$ & $\mathrm{U}(2,3)$ & $\mathrm{U}(1,3)$ & $\mathrm{U}(1,2)$ \\
\hline$-\ldots$ & ----- & ----- & ---- & ----- & ----- & ----- \\
\hline Mn & $0.0287(4)$ & $0.0254(3)$ & $0.0341(4)$ & $-0.0015(2)$ & $0.0046(3)$ & $0.0029(2)$ \\
\hline $\mathrm{O} 1$ & $0.046(2)$ & $0.060(2)$ & $0.054(2)$ & $-0.0130(16)$ & $0.0168(19)$ & $-0.0127(17)$ \\
\hline O1A & $0.0296(16)$ & $0.0297(13)$ & $0.0438(17)$ & $-0.0084(11)$ & $0.0058(14)$ & $0.0012(12)$ \\
\hline $1 B$ & $0.0269(16)$ & $0.0280(13)$ & $0.0453(18)$ & $-0.0056(11)$ & $0.0012(15)$ & $0.0010(12)$ \\
\hline $\mathrm{O} 2$ & $0.052(2)$ & $0.0471(18)$ & $0.052(2)$ & $0.0037(15)$ & $0.006(2)$ & $-0.0017(17)$ \\
\hline $\mathrm{O} 2 \mathrm{~A}$ & $0.043(2)$ & $0.0548(19)$ & $0.041(2)$ & $0.0008(14)$ & $-0.0063(18)$ & $0.0193(16)$ \\
\hline $\mathrm{O} 2 \mathrm{~B}$ & $0.049(2)$ & $0.0311(14)$ & $0.0486(18)$ & $0.0040(12)$ & $0.0246(17)$ & $0.0016(13)$ \\
\hline N1A & $0.041(2)$ & $0.0301(16)$ & $0.0315(19)$ & $-0.0003(13)$ & $0.0045(18)$ & $0.0030(15)$ \\
\hline N1B & $0.040(2)$ & $0.0229(15)$ & $0.0304(19)$ & $-0.0017(12)$ & $0.0039(17)$ & $0.0029(14)$ \\
\hline $\mathrm{C} 1$ & $0.052(3)$ & $0.039(2)$ & $0.035(3)$ & $-0.0023(17)$ & $0.009(3)$ & $-0.009(2)$ \\
\hline $\mathrm{C} 1 \mathrm{~A}$ & $0.035(2)$ & $0.0233(16)$ & $0.035(2)$ & $0.0008(14)$ & $0.012(2)$ & $-0.0001(16)$ \\
\hline $\mathrm{C} 1 \mathrm{~B}$ & $0.027(2)$ & $0.0274(17)$ & $0.025(2)$ & $-0.0044(13)$ & $0.0006(18)$ & $0.0017(16)$ \\
\hline $\mathrm{C} 2$ & $0.091(5)$ & $0.063(3)$ & $0.047(3)$ & $0.011(2)$ & $0.018(3)$ & $-0.010(3)$ \\
\hline $\mathrm{C} 2 \mathrm{~A}$ & $0.047(3)$ & $0.035(2)$ & $0.036(2)$ & $-0.0028(17)$ & $0.007(2)$ & $-0.0028(19$ \\
\hline $\mathrm{C} 2 \mathrm{~B}$ & $0.040(3)$ & $0.0290(18)$ & $0.031(2)$ & $-0.0027(15)$ & $0.003(2)$ & $-0.0068(18)$ \\
\hline C3A & $0.078(4)$ & $0.038(2)$ & $0.049(3)$ & $-0.009(2)$ & $0.031(3)$ & $0.007(2)$ \\
\hline C3B & $0.047(3)$ & $0.0228(17)$ & $0.034(2)$ & $-0.0009(14)$ & $0.005(2)$ & $0.0017(17)$ \\
\hline $\mathrm{C} 4 \mathrm{~A}$ & $0.073(4)$ & $0.051(3)$ & $0.055(3)$ & $-0.004(2)$ & $0.027(3)$ & $0.022(3)$ \\
\hline$C 4 B$ & $0.048(3)$ & $0.0280(18)$ & $0.030(2)$ & $-0.0002(15)$ & $0.004(2)$ & $0.0111(18)$ \\
\hline C5A & $0.046(3)$ & $0.048(2)$ & $0.050(3)$ & $0.005(2)$ & $0.016(3)$ & $0.017(2)$ \\
\hline C5B & $0.032(2)$ & $0.038(2)$ & $0.026(2)$ & $-0.0001(15)$ & $0.004(2)$ & $0.0039(17)$ \\
\hline $\mathrm{C} 6 \mathrm{~A}$ & $0.038(2)$ & $0.0285(18)$ & $0.035(2)$ & $0.0048(15)$ & $0.012(2)$ & $0.0067(17)$ \\
\hline $\mathrm{C} 6 \mathrm{~B}$ & $0.033(2)$ & $0.0226(16)$ & $0.023(2)$ & $-0.0012(13)$ & $0.0023(19)$ & $0.0029(15)$ \\
\hline C7A & $0.035(3)$ & $0.0313(18)$ & $0.033(2)$ & $0.0062(16)$ & $0.006(2)$ & $0.0019(18)$ \\
\hline C7B & $0.031(2)$ & $0.0289(17)$ & $0.0199(19)$ & $0.0000(14)$ & $-0.0027(18)$ & $0.0020(16)$ \\
\hline $\mathrm{C} 8$ & $0.044(3)$ & $0.063(3)$ & $0.042(3)$ & $0.003(2)$ & $-0.013(3)$ & $0.006(3)$ \\
\hline
\end{tabular}




$\begin{array}{lcccccc}\text { C8B } & 0.043(3) & 0.044(2) & 0.041(3) & 0.0045(19) & 0.017(2) & -0.009(2) \\ \text { C9A } & 0.073(4) & 0.060(3) & 0.035(3) & -0.001(2) & -0.007(3) & 0.009(3) \\ \text { C9B } & 0.064(3) & 0.035(2) & 0.039(3) & 0.0061(17) & 0.014(3) & -0.001(2) \\ \text { C10A } & 0.056(3) & 0.049(3) & 0.041(3) & -0.010(2) & 0.000(3) & 0.009(2) \\ \text { C10B } & 0.060(3) & 0.0263(19) & 0.039(3) & 0.0039(16) & 0.012(2) & 0.0025(19)\end{array}$

The Temperature Factor has the Form of $\operatorname{Exp}(-T)$ Where $T=8 *(P i * * 2) * U *(\operatorname{Sin}($ Theta $) /$ Lambda $) * * 2$ for Isotropic Atoms $T=2 *(P i * * 2) * \operatorname{Sumij}(h(i) * h(j) * U(i, j) * A s t a r(i) * A s t a r(j))$, for Anisotropic Atoms. Astar(i) are Reciprocal Axial Lengths and $h(i)$ are the Reflection Indices. 

Table S5 - Bond Distances (Angstrom)
for: 9

\begin{tabular}{|c|c|c|c|c|c|}
\hline Mn & -01 & $2.262(4)$ & $\mathrm{C} 4 \mathrm{~A}$ & $-\mathrm{C} 5 \mathrm{~A}$ & $1.369(7)$ \\
\hline Mn & $-01 A$ & $1.867(4)$ & $\mathrm{C} 4 \mathrm{~B}$ & $-C 5 B$ & $1.396(6)$ \\
\hline Mn & $-01 B$ & $1.861(4)$ & C5A & $-\mathrm{C} 6 \mathrm{~A}$ & $1.399(8)$ \\
\hline Mn & -02 & $2.113(4)$ & C5B & $-C 6 B$ & $1.403(6)$ \\
\hline Mn & $-\mathrm{N} 1 \mathrm{~A}$ & $2.107(4)$ & $\mathrm{C} 6 \mathrm{~A}$ & $-C 7 A$ & $1.465(6)$ \\
\hline Mn & $-\mathrm{N} 1 \mathrm{~B}$ & $2.086(5)$ & $\mathrm{C} 6 \mathrm{~B}$ & $-C 7 B$ & $1.477(5)$ \\
\hline $\mathrm{O} 1$ & $-\mathrm{C} 1$ & $1.258(7)$ & C8B & $-C 9 B$ & $1.489(7)$ \\
\hline $\mathrm{O} 1 \mathrm{~A}$ & $-C 1 A$ & $1.327(5)$ & C9A & $-\mathrm{C} 10 \mathrm{~A}$ & $1.522(7)$ \\
\hline $\mathrm{O} 1 \mathrm{~B}$ & $-\mathrm{C} 1 \mathrm{~B}$ & $1.331(5)$ & $\mathrm{C} 9 \mathrm{~B}$ & $-\mathrm{C} 10 \mathrm{~B}$ & $1.519(8)$ \\
\hline $\mathrm{O} 2$ & $-\mathrm{C} 1$ & $1.260(6)$ & $\mathrm{C} 2$ & $-\mathrm{H} 2 \mathrm{~A}$ & 0.9602 \\
\hline $\mathrm{O} 2 \mathrm{~A}$ & $-C 7 A$ & $1.349(6)$ & $\mathrm{C} 2$ & $-\mathrm{H} 2 \mathrm{~B}$ & 0.9602 \\
\hline $\mathrm{O} 2 \mathrm{~A}$ & $-C 8 A$ & $1.445(6)$ & $\mathrm{C} 2$ & $-\mathrm{H} 2 \mathrm{C}$ & 0.9603 \\
\hline $\mathrm{O} 2 \mathrm{~B}$ & $-C 7 B$ & $1.340(5)$ & $\mathrm{C} 2 \mathrm{~A}$ & $-\mathrm{H} 2 \mathrm{AA}$ & 0.9298 \\
\hline $\mathrm{O} 2 \mathrm{~B}$ & $-C 8 B$ & $1.460(5)$ & $\mathrm{C} 2 \mathrm{~B}$ & $-\mathrm{H} 2 \mathrm{BA}$ & 0.9288 \\
\hline $\mathrm{N} 1 \mathrm{~A}$ & $-C 7 A$ & $1.288(6)$ & C3A & $-\mathrm{H} 3 \mathrm{AA}$ & 0.9295 \\
\hline N1A & $-\mathrm{C} 10 \mathrm{~A}$ & $1.482(6)$ & C $3 B$ & $-\mathrm{H} 3 \mathrm{BA}$ & 0.9295 \\
\hline N1B & $-C 7 B$ & $1.287(6)$ & $C 4 \mathrm{~A}$ & $-\mathrm{H} 4 \mathrm{AA}$ & 0.9299 \\
\hline N1B & $-\mathrm{C} 10 \mathrm{~B}$ & $1.485(5)$ & $\mathrm{C} 4 \mathrm{~B}$ & $-\mathrm{H} 4 \mathrm{BA}$ & 0.9306 \\
\hline $\mathrm{C} 1$ & $-\mathrm{C} 2$ & $1.487(8)$ & C5A & $-\mathrm{H} 5 \mathrm{AA}$ & 0.9310 \\
\hline $\mathrm{C} 1 \mathrm{~A}$ & $-\mathrm{C} 2 \mathrm{~A}$ & $1.403(6)$ & C5B & $-\mathrm{H} 5 \mathrm{BA}$ & 0.9294 \\
\hline $\mathrm{C} 1 \mathrm{~A}$ & $-C 6 A$ & $1.424(7)$ & C8A & $-\mathrm{H} 8 \mathrm{AA}$ & 0.9703 \\
\hline $\mathrm{C} 1 \mathrm{~B}$ & $-C 2 B$ & $1.411(5)$ & C8A & $-\mathrm{H} 8 \mathrm{AB}$ & 0.9700 \\
\hline $\mathrm{C} 1 \mathrm{~B}$ & $-\mathrm{C} 6 \mathrm{~B}$ & $1.417(6)$ & C8B & $-\mathrm{H} 8 \mathrm{C}$ & 0.9702 \\
\hline $\mathrm{C} 2 \mathrm{~A}$ & $-C 3 A$ & $1.371(8)$ & C8B & $-H 8 D$ & 0.9712 \\
\hline $\mathrm{C} 2 \mathrm{~B}$ & $-C 3 B$ & $1.388(7)$ & C9A & $-\mathrm{H} 9 \mathrm{AA}$ & 0.9707 \\
\hline C3A & $-C 4 A$ & $1.386(9)$ & C9A & $-\mathrm{H} 9 \mathrm{AB}$ & 0.9708 \\
\hline C3B & $-C 4 B$ & $1.365(8)$ & C9B & $-\mathrm{H} 9 \mathrm{BA}$ & 0.9693 \\
\hline
\end{tabular}




$\begin{array}{llllll}\mathrm{C} 9 \mathrm{~B} & -\mathrm{H} 9 \mathrm{BB} & 0.9704 & \mathrm{C} 10 \mathrm{~B} & -\mathrm{H} 10 \mathrm{BA} & 0.9703 \\ \mathrm{C} 10 \mathrm{~A} & -\mathrm{H} 10 \mathrm{~A} & 0.9697 & \mathrm{C} 10 \mathrm{~B} & -\mathrm{H} 10 \mathrm{BB} & 0.9702 \\ \mathrm{C} 10 \mathrm{~A} & -\mathrm{H} 10 \mathrm{~B} & 0.9699 & & & \end{array}$




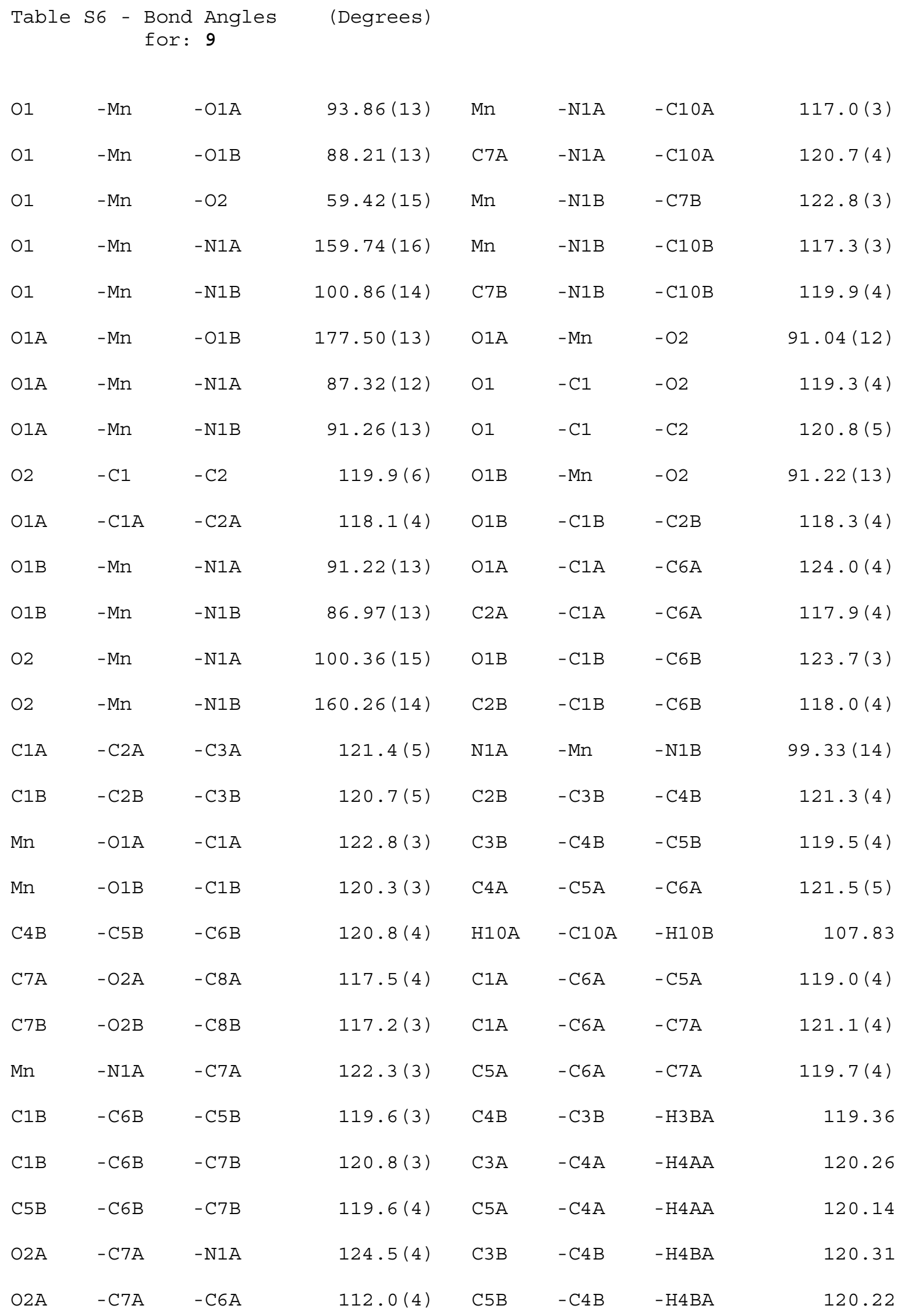




\begin{tabular}{|c|c|c|c|c|c|c|c|}
\hline $\mathrm{N} 1 \mathrm{~A}$ & $-C 7 A$ & $-\mathrm{C} 6 \mathrm{~A}$ & $123.5(4)$ & $\mathrm{C} 4 \mathrm{~A}$ & $-\mathrm{C} 5 \mathrm{~A}$ & $-\mathrm{H} 5 \mathrm{AA}$ & 119.22 \\
\hline $\mathrm{O} 2 \mathrm{~B}$ & $-C 7 B$ & $-\mathrm{N} 1 \mathrm{~B}$ & $125.8(4)$ & $\mathrm{C} 6 \mathrm{~A}$ & $-\mathrm{C} 5 \mathrm{~A}$ & $-\mathrm{H} 5 \mathrm{AA}$ & 119.26 \\
\hline $\mathrm{O} 2 \mathrm{~B}$ & $-C 7 B$ & $-C 6 B$ & $111.8(3)$ & $\mathrm{C} 4 \mathrm{~B}$ & $-\mathrm{C} 5 \mathrm{~B}$ & $-\mathrm{H} 5 \mathrm{BA}$ & 119.59 \\
\hline $\mathrm{N} 1 \mathrm{~B}$ & $-C 7 B$ & $-\mathrm{C} 6 \mathrm{~B}$ & $122.4(4)$ & $\mathrm{C} 6 \mathrm{~B}$ & $-\mathrm{C} 5 \mathrm{~B}$ & $-\mathrm{H} 5 \mathrm{BA}$ & 119.58 \\
\hline $\mathrm{O} 2 \mathrm{~A}$ & $-C 8 A$ & $-C 9 A$ & $111.6(5)$ & $\mathrm{O} 2 \mathrm{~A}$ & $-C 8 A$ & $-\mathrm{H} 8 \mathrm{AA}$ & 109.32 \\
\hline $\mathrm{O} 2 \mathrm{~B}$ & $-C 8 B$ & $-C 9 B$ & $110.2(4)$ & $\mathrm{O} 2 \mathrm{~A}$ & $-C 8 A$ & $-\mathrm{H} 8 \mathrm{AB}$ & 109.25 \\
\hline $\mathrm{C} 8 \mathrm{~A}$ & $-\mathrm{C} 9 \mathrm{~A}$ & $-\mathrm{C} 10 \mathrm{~A}$ & $108.5(4)$ & C9A & $-C 8 A$ & $-\mathrm{H} 8 \mathrm{AA}$ & 109.33 \\
\hline $\mathrm{C} 8 \mathrm{~B}$ & $-C 9 B$ & $-\mathrm{C} 10 \mathrm{~B}$ & $107.8(4)$ & C9A & $-C 8 A$ & $-\mathrm{H} 8 \mathrm{AB}$ & 109.33 \\
\hline $\mathrm{N} 1 \mathrm{~A}$ & $-\mathrm{C} 10 \mathrm{~A}$ & $-C 9 A$ & $111.9(4)$ & H8AA & $-C 8 A$ & $-\mathrm{H} 8 \mathrm{AB}$ & 107.93 \\
\hline N1B & $-\mathrm{C} 10 \mathrm{~B}$ & $-C 9 B$ & $112.0(3)$ & $\mathrm{O} 2 \mathrm{~B}$ & $-C 8 B$ & $-\mathrm{H} 8 \mathrm{C}$ & 109.64 \\
\hline $\mathrm{C} 1$ & $-\mathrm{C} 2$ & $-\mathrm{H} 2 \mathrm{~A}$ & 109.49 & $\mathrm{O} 2 \mathrm{~B}$ & $-C 8 B$ & $-\mathrm{H} 8 \mathrm{D}$ & 109.57 \\
\hline $\mathrm{C} 1$ & $-\mathrm{C} 2$ & $-\mathrm{H} 2 \mathrm{~B}$ & 109.53 & $\mathrm{C} 9 \mathrm{~B}$ & $-C 8 B$ & $-\mathrm{H} 8 \mathrm{C}$ & 109.64 \\
\hline $\mathrm{C} 1$ & $-\mathrm{C} 2$ & $-\mathrm{H} 2 \mathrm{C}$ & 109.50 & $\mathrm{C} 9 \mathrm{~B}$ & $-C 8 B$ & $-\mathrm{H} 8 \mathrm{D}$ & 109.62 \\
\hline $\mathrm{H} 2 \mathrm{~A}$ & $-\mathrm{C} 2$ & $-\mathrm{H} 2 \mathrm{~B}$ & 109.44 & $\mathrm{H} 8 \mathrm{C}$ & $-C 8 B$ & $-\mathrm{H} 8 \mathrm{D}$ & 108.15 \\
\hline $\mathrm{H} 2 \mathrm{~A}$ & $-\mathrm{C} 2$ & $-\mathrm{H} 2 \mathrm{C}$ & 109.43 & $\mathrm{C} 8 \mathrm{~A}$ & $-\mathrm{C} 9 \mathrm{~A}$ & $-\mathrm{H} 9 \mathrm{AA}$ & 110.00 \\
\hline $\mathrm{H} 2 \mathrm{~B}$ & $-\mathrm{C} 2$ & $-\mathrm{H} 2 \mathrm{C}$ & 109.44 & C8A & $-\mathrm{C} 9 \mathrm{~A}$ & $-\mathrm{H} 9 \mathrm{AB}$ & 109.98 \\
\hline $\mathrm{C} 1 \mathrm{~A}$ & $-\mathrm{C} 2 \mathrm{~A}$ & $-\mathrm{H} 2 \mathrm{AA}$ & 119.27 & $\mathrm{C} 10 \mathrm{~A}$ & $-\mathrm{C} 9 \mathrm{~A}$ & $-\mathrm{H} 9 \mathrm{AA}$ & 110.02 \\
\hline C3A & $-\mathrm{C} 2 \mathrm{~A}$ & $-\mathrm{H} 2 \mathrm{AA}$ & 119.38 & $\mathrm{C} 10 \mathrm{~A}$ & $-\mathrm{C} 9 \mathrm{~A}$ & $-\mathrm{H} 9 \mathrm{AB}$ & 110.01 \\
\hline $\mathrm{C} 1 \mathrm{~B}$ & $-\mathrm{C} 2 \mathrm{~B}$ & $-\mathrm{H} 2 \mathrm{BA}$ & 119.64 & H9AA & $-\mathrm{C} 9 \mathrm{~A}$ & $-\mathrm{H} 9 \mathrm{AB}$ & 108.30 \\
\hline C3B & $-\mathrm{C} 2 \mathrm{~B}$ & $-\mathrm{H} 2 \mathrm{BA}$ & 119.62 & $\mathrm{C} 8 \mathrm{~B}$ & $-C 9 B$ & $-\mathrm{H} 9 \mathrm{BA}$ & 110.11 \\
\hline $\mathrm{C} 2 \mathrm{~A}$ & $-C 3 A$ & $-\mathrm{H} 3 \mathrm{AA}$ & 119.72 & $\mathrm{C} 8 \mathrm{~B}$ & $-C 9 B$ & $-\mathrm{H} 9 \mathrm{BB}$ & 110.09 \\
\hline $\mathrm{C} 4 \mathrm{~A}$ & $-C 3 A$ & $-\mathrm{H} 3 \mathrm{AA}$ & 119.68 & $\mathrm{C} 10 \mathrm{~B}$ & $-C 9 B$ & $-\mathrm{H} 9 \mathrm{BA}$ & 110.18 \\
\hline $\mathrm{C} 2 \mathrm{~B}$ & $-C 3 B$ & $-\mathrm{H} 3 \mathrm{BA}$ & 119.35 & $\mathrm{C} 10 \mathrm{~B}$ & $-C 9 B$ & $-\mathrm{H} 9 \mathrm{BB}$ & 110.15 \\
\hline H9BA & $-C 9 B$ & $-\mathrm{H} 9 \mathrm{BB}$ & 108.49 & $\mathrm{~N} 1 \mathrm{~B}$ & $-\mathrm{C} 10 \mathrm{~B}$ & $-\mathrm{H} 10 \mathrm{BA}$ & 109.24 \\
\hline $\mathrm{N} 1 \mathrm{~A}$ & $-C 10 A$ & $-\mathrm{H} 10 \mathrm{~A}$ & 109.30 & $\mathrm{~N} 1 \mathrm{~B}$ & $-\mathrm{C} 10 \mathrm{~B}$ & $-\mathrm{H} 10 \mathrm{BB}$ & 109.21 \\
\hline N1A & $-\mathrm{C} 10 \mathrm{~A}$ & $-\mathrm{H} 10 \mathrm{~B}$ & 109.22 & C9B & $-\mathrm{C} 10 \mathrm{~B}$ & $-\mathrm{H} 10 \mathrm{BA}$ & 109.22 \\
\hline C9A & $-\mathrm{C} 10 \mathrm{~A}$ & $-\mathrm{H} 10 \mathrm{~A}$ & 109.22 & C9B & $-\mathrm{C} 10 \mathrm{~B}$ & $-\mathrm{H} 10 \mathrm{BB}$ & 109.15 \\
\hline C9A & $-\mathrm{C} 10 \mathrm{~A}$ & $-\mathrm{H} 10 \mathrm{~B}$ & 109.28 & $\mathrm{H} 10 \mathrm{BA}$ & $-\mathrm{C} 10 \mathrm{~B}$ & - H1 0BB & 107.92 \\
\hline
\end{tabular}









\begin{tabular}{|c|c|c|c|c|}
\hline Mn & $-01 \mathrm{~A}$ & $-C 1 A$ & $-\mathrm{C} 2 \mathrm{~A}$ & $144.1(3)$ \\
\hline Mn & $-01 \mathrm{~A}$ & $-C 1 A$ & $-C 6 A$ & $-37.5(5)$ \\
\hline Mn & $-01 B$ & $-\mathrm{C} 1 \mathrm{~B}$ & $-C 2 B$ & $136.2(3)$ \\
\hline Mn & $-01 B$ & $-C 1 B$ & $-C 6 B$ & $-44.1(5)$ \\
\hline C8A & $-02 \mathrm{~A}$ & $-C 7 A$ & $-\mathrm{N} 1 \mathrm{~A}$ & $5.6(7)$ \\
\hline $\mathrm{C} 8 \mathrm{~A}$ & $-02 \mathrm{~A}$ & $-C 7 A$ & $-\mathrm{C} 6 \mathrm{~A}$ & $-175.4(4)$ \\
\hline C7A & $-02 \mathrm{~A}$ & $-C 8 A$ & $-\mathrm{C} 9 \mathrm{~A}$ & $-38.5(6)$ \\
\hline C8B & $-\mathrm{O} 2 \mathrm{~B}$ & $-C 7 B$ & $-N 1 B$ & $6.5(5)$ \\
\hline C8B & $-02 \mathrm{~B}$ & $-C 7 B$ & $-C 6 B$ & $-173.2(3)$ \\
\hline C7B & $-\mathrm{O} 2 \mathrm{~B}$ & $-C 8 B$ & $-C 9 B$ & $-39.5(5)$ \\
\hline Mn & $-\mathrm{N} 1 \mathrm{~A}$ & $-\mathrm{C} 10 \mathrm{~A}$ & - C9A & $-165.4(4)$ \\
\hline C7A & $-\mathrm{N} 1 \mathrm{~A}$ & $-\mathrm{C} 10 \mathrm{~A}$ & - C9A & $15.7(7)$ \\
\hline $\mathrm{Mn}$ & $-\mathrm{N} 1 \mathrm{~A}$ & $-C 7 A$ & $-\mathrm{C} 6 \mathrm{~A}$ & $7.9(6)$ \\
\hline $\mathrm{C} 10 \mathrm{~A}$ & $-\mathrm{N} 1 \mathrm{~A}$ & $-C 7 A$ & $-02 \mathrm{~A}$ & $5.7(7)$ \\
\hline Mn & $-\mathrm{N} 1 \mathrm{~A}$ & $-C 7 A$ & $-02 \mathrm{~A}$ & $-173.2(3)$ \\
\hline $\mathrm{C} 10 \mathrm{~A}$ & $-\mathrm{N} 1 \mathrm{~A}$ & $-C 7 A$ & $-\mathrm{C} 6 \mathrm{~A}$ & $-173.2(4)$ \\
\hline Mn & $-N 1 B$ & $-C 7 B$ & $-02 \mathrm{~B}$ & $-173.1(3)$ \\
\hline Mn & $-\mathrm{N} 1 \mathrm{~B}$ & $-C 7 B$ & $-C 6 B$ & $6.5(5)$ \\
\hline $\mathrm{C} 10 \mathrm{~B}$ & $-\mathrm{N} 1 \mathrm{~B}$ & $-\mathrm{C} 7 \mathrm{~B}$ & $-\mathrm{O} 2 \mathrm{~B}$ & $4.8(5)$ \\
\hline $\mathrm{C} 10 \mathrm{~B}$ & $-\mathrm{N} 1 \mathrm{~B}$ & $-C 7 B$ & $-\mathrm{C} 6 \mathrm{~B}$ & $-175.5(4)$ \\
\hline Mn & $-N 1 B$ & $-\mathrm{C} 10 \mathrm{~B}$ & $-C 9 B$ & $-164.5(3)$ \\
\hline C7B & $-\mathrm{N} 1 \mathrm{~B}$ & $-\mathrm{C} 10 \mathrm{~B}$ & $-C 9 B$ & $17.5(6)$ \\
\hline $\mathrm{C} 6 \mathrm{~A}$ & $-\mathrm{C} 1 \mathrm{~A}$ & $-\mathrm{C} 2 \mathrm{~A}$ & $-C 3 A$ & $1.1(6)$ \\
\hline $\mathrm{O} 1 \mathrm{~A}$ & $-\mathrm{C} 1 \mathrm{~A}$ & $-\mathrm{C} 6 \mathrm{~A}$ & $-\mathrm{C} 5 \mathrm{~A}$ & $-179.3(4)$ \\
\hline $\mathrm{O} 1 \mathrm{~A}$ & $-\mathrm{C} 1 \mathrm{~A}$ & $-\mathrm{C} 2 \mathrm{~A}$ & $-C 3 A$ & $179.6(4)$ \\
\hline $\mathrm{C} 2 \mathrm{~A}$ & $-\mathrm{C} 1 \mathrm{~A}$ & $-C 6 A$ & $-C 7 A$ & $175.0(4)$ \\
\hline O1A & $-\mathrm{C} 1 \mathrm{~A}$ & $-\mathrm{C} 6 \mathrm{~A}$ & $-C 7 A$ & $-3.4(6)$ \\
\hline $\mathrm{C} 2 \mathrm{~A}$ & $-\mathrm{C} 1$ & $-C 6 A$ & $-\mathrm{C} 5 \mathrm{~A}$ & $-0.9(6)$ \\
\hline
\end{tabular}




\begin{tabular}{|c|c|c|c|c|}
\hline $\mathrm{O} 1 \mathrm{~B}$ & $-\mathrm{C} 1 \mathrm{~B}$ & $-\mathrm{C} 2 \mathrm{~B}$ & $-C 3 B$ & $177.6(4)$ \\
\hline $\mathrm{C} 6 \mathrm{~B}$ & $-C 1 B$ & $-\mathrm{C} 2 \mathrm{~B}$ & $-C 3 B$ & $-2.1(6)$ \\
\hline O1B & $-\mathrm{C} 1 \mathrm{~B}$ & $-\mathrm{C} 6 \mathrm{~B}$ & $-C 5 B$ & $-178.0(4)$ \\
\hline $\mathrm{O} 1 \mathrm{~B}$ & $-\mathrm{C} 1 \mathrm{~B}$ & $-C 6 B$ & $-C 7 B$ & $0.5(6)$ \\
\hline $\mathrm{C} 2 \mathrm{~B}$ & $-\mathrm{C} 1 \mathrm{~B}$ & $-\mathrm{C} 6 \mathrm{~B}$ & $-C 5 B$ & $1.7(6)$ \\
\hline $\mathrm{C} 2 \mathrm{~B}$ & $-\mathrm{C} 1 \mathrm{~B}$ & $-\mathrm{C} 6 \mathrm{~B}$ & $-C 7 B$ & $-179.8(4)$ \\
\hline $\mathrm{C} 1 \mathrm{~A}$ & $-\mathrm{C} 2 \mathrm{~A}$ & $-C 3 A$ & $-C 4 A$ & $-0.4(7)$ \\
\hline $\mathrm{C} 1 \mathrm{~B}$ & $-\mathrm{C} 2 \mathrm{~B}$ & $-C 3 B$ & $-C 4 B$ & $0.5(7)$ \\
\hline $\mathrm{C} 2 \mathrm{~A}$ & $-C 3 A$ & $-\mathrm{C} 4 \mathrm{~A}$ & $-C 5 A$ & $-0.5(8)$ \\
\hline $\mathrm{C} 2 \mathrm{~B}$ & $-C 3 B$ & $-C 4 B$ & $-C 5 B$ & $1.6(7)$ \\
\hline C3A & $-C 4 A$ & $-\mathrm{C} 5 \mathrm{~A}$ & $-C 6 \mathrm{~A}$ & $0.8(8)$ \\
\hline C3B & $-C 4 B$ & $-C 5 B$ & $-C 6 B$ & $-2.0(7)$ \\
\hline $\mathrm{C} 4 \mathrm{~A}$ & $-C 5 A$ & $-C 6 A$ & $-C 1 A$ & $0.0(7)$ \\
\hline $\mathrm{C} 4 \mathrm{~A}$ & $-C 5 A$ & $-\mathrm{C} 6 \mathrm{~A}$ & $-C 7 A$ & $-176.0(5)$ \\
\hline $\mathrm{C} 4 \mathrm{~B}$ & $-C 5 B$ & $-\mathrm{C} 6 \mathrm{~B}$ & $-\mathrm{C} 1 \mathrm{~B}$ & $0.3(7)$ \\
\hline $\mathrm{C} 4 \mathrm{~B}$ & $-C 5 B$ & $-\mathrm{C} 6 \mathrm{~B}$ & $-C 7 B$ & $-178.2(4)$ \\
\hline $\mathrm{C} 1 \mathrm{~A}$ & $-C 6 A$ & $-C 7 A$ & $-\mathrm{N} 1 \mathrm{~A}$ & $17.0(7)$ \\
\hline $\mathrm{C} 5 \mathrm{~A}$ & $-C 6 A$ & $-C 7 A$ & $-02 \mathrm{~A}$ & $13.9(6)$ \\
\hline C5A & $-C 6 A$ & $-C 7 A$ & $-\mathrm{N} 1 \mathrm{~A}$ & $-167.1(4)$ \\
\hline $\mathrm{C} 1 \mathrm{~A}$ & $-C 6 A$ & $-C 7 A$ & $-02 \mathrm{~A}$ & $-162.0(4)$ \\
\hline $\mathrm{C} 1 \mathrm{~B}$ & $-\mathrm{C} 6 \mathrm{~B}$ & $-C 7 B$ & $-02 B$ & $-162.6(4)$ \\
\hline $\mathrm{C} 1 \mathrm{~B}$ & $-C 6 B$ & $-C 7 B$ & $-\mathrm{N} 1 \mathrm{~B}$ & $17.7(6)$ \\
\hline C5B & $-\mathrm{C} 6 \mathrm{~B}$ & $-C 7 B$ & $-02 B$ & $15.9(5)$ \\
\hline C5B & $-\mathrm{C} 6 \mathrm{~B}$ & $-C 7 B$ & $-N 1 B$ & $-163.8(4)$ \\
\hline $\mathrm{O} 2 \mathrm{~A}$ & $-C 8 A$ & $-C 9 A$ & $-\mathrm{C} 10 \mathrm{~A}$ & $57.1(5)$ \\
\hline $\mathrm{O} 2 \mathrm{~B}$ & $-C 8 B$ & $-C 9 B$ & $-\mathrm{C} 10 \mathrm{~B}$ & $58.6(4)$ \\
\hline C8A & $-C 9 A$ & $-\mathrm{C} 10 \mathrm{~A}$ & $-\mathrm{N} 1 \mathrm{~A}$ & $-45.8(6)$ \\
\hline C8. & $-\mathrm{C} 9 \mathrm{~B}$ & $-\mathrm{C} 10 \mathrm{~B}$ & $-\mathrm{N} 1 \mathrm{~B}$ & $-47.9(5)$ \\
\hline
\end{tabular}




\begin{tabular}{|c|c|c|c|c|c|}
\hline O1 &. $\mathrm{C} 6 \mathrm{~B}$ & $3.419(6)$ & C8A & $.02 A_{-} e$ & $3.376(7)$ \\
\hline $\mathrm{O} 1$ & . $C 4 B_{-}$ & $3.413(8)$ & C8A & . $\mathrm{C}_{2} \mathrm{~B}_{-} \mathrm{i}$ & $3.558(8)$ \\
\hline $\mathrm{O} 2$ & . C5A_C & $3.409(8)$ & $\mathrm{C} 10 \mathrm{~A}$ & . C3A_k & $3.490(8)$ \\
\hline $\mathrm{O} 2 \mathrm{~A}$ & . C8A_e & $3.376(7)$ & $\mathrm{C} 1 \mathrm{~A}$ & .H3BA_b & 2.8670 \\
\hline $\mathrm{O} 2 \mathrm{~B}$ & $.02 B \_9$ & $3.113(5)$ & $\mathrm{C} 2 \mathrm{~A}$ &. $\mathrm{H} 10 \mathrm{~B} \_\mathrm{h}$ & 3.0673 \\
\hline O1 & . H4BA_a & 2.5694 & $\mathrm{C} 2 \mathrm{~B}$ & .H8AA_i & 2.9787 \\
\hline O1A & . H10BA & 2.7389 & $\mathrm{C} 2 \mathrm{~B}$ & .H8D_f & 3.0112 \\
\hline O1A & . $\mathrm{H} 10 \mathrm{BB}$ & 2.8522 & C3A &. $\mathrm{H} 10 \mathrm{~B} \_\mathrm{h}$ & 2.8149 \\
\hline O1A & .H3BA_b & 2.7951 & C3B &.$H 8 D_{-} \mathrm{f}$ & 2.8282 \\
\hline O1A & . H5BA_a & 2.6952 & C3B & . H2A_j & 2.9588 \\
\hline O1B & . H10B & 2.8572 & $\mathrm{C} 4 \mathrm{~B}$ &. $\mathrm{H} 2 \mathrm{~A} \_j$ & 3.0531 \\
\hline O1B & . H10A & 2.7358 & C5B & . H10BA_f & 2.8143 \\
\hline $\mathrm{O} 2$ & .H5AA_C & 2.6231 & C5B & . H8C_9 & 2.8142 \\
\hline $\mathrm{O} 2 \mathrm{~A}$ & . H2C_d & 2.8237 & C7A & .H4AA_C & 2.8848 \\
\hline $\mathrm{O} 2 \mathrm{~A}$ & . H5AA & 2.3541 & C7A & . H9AA & 2.9569 \\
\hline $\mathrm{O} 2 \mathrm{~A}$ & .H8AA_e & 2.8315 & C7B & . H9BA & 2.9301 \\
\hline $\mathrm{O} 2 \mathrm{~B}$ & . H5BA & 2.3699 & C8A & . $\mathrm{H} 2 \mathrm{C} \_\mathrm{d}$ & 3.0475 \\
\hline $\mathrm{O} 2 \mathrm{~B}$ & .H2AA_f & 2.8837 & $\mathrm{C} 8 \mathrm{~A}$ & . $\mathrm{H} 2 \mathrm{BA} \_i$ & 3.0754 \\
\hline $\mathrm{O} 2 \mathrm{~B}$ & . H8C_g & 2.9176 & C9B & . $\mathrm{H} 2 \mathrm{~A} \_\mathrm{k}$ & 3.0718 \\
\hline $\mathrm{N} 1 \mathrm{~B}$ & . C4B_a & $3.417(7)$ & $\mathrm{C} 10 \mathrm{~B}$ & . H3BA_b & 2.9846 \\
\hline $\mathrm{C} 2 \mathrm{~B}$ & . C8A_i & $3.558(8)$ & $\mathrm{C} 10 \mathrm{~B}$ & . $\mathrm{H} 2 \mathrm{~A} \_\mathrm{k}$ & 3.0767 \\
\hline C3A & .C10A_h & $3.490(8)$ & $\mathrm{H} 2 \mathrm{AA}$ & $.02 B \_a$ & 2.8837 \\
\hline C4B & . C7B_f & $3.482(7)$ & H3AA & . $\mathrm{H} 2 \mathrm{~B} \_\mathrm{b}$ & 2.5094 \\
\hline C4B &.$N 1 B \_f$ & $3.417(7)$ & $\mathrm{H} 2 \mathrm{~A}$ &. $\mathrm{C}_{3} \mathrm{~B}_{-} 1$ & 2.9588 \\
\hline $\mathrm{C} 4 \mathrm{~B}$ & $.01 \_\mathrm{f}$ & $3.413(8)$ & $\mathrm{H} 2 \mathrm{~A}$ & . $\mathrm{C}_{4} \mathrm{~B}_{-} 1$ & 3.0531 \\
\hline
\end{tabular}




\begin{tabular}{|c|c|c|c|c|c|}
\hline C5A & $.02 \_d$ & $3.409(8)$ & $\mathrm{H} 2 \mathrm{~A}$ & . $C 9 B_{-} h$ & 3.0718 \\
\hline C6B & .01 & $3.419(6)$ & $\mathrm{H} 2 \mathrm{~A}$ & . $\mathrm{C} 10 \mathrm{~B} \_\mathrm{h}$ & 3.0767 \\
\hline C7B & . C4B_a & $3.482(7)$ & $\mathrm{H} 2 \mathrm{~A}$ & .H10BB_h & 2.4337 \\
\hline $\mathrm{H} 2 \mathrm{~B}$ & .H3AA_m & 2.5094 & H3BA & . $\mathrm{C} 10 \mathrm{~B} \_\mathrm{m}$ & 2.9846 \\
\hline $\mathrm{H} 2 \mathrm{C}$ & $.02 A_{-} \mathrm{C}$ & 2.8237 & H3BA & .H10BA_m & 2.4932 \\
\hline $\mathrm{H} 2 \mathrm{C}$ &.$C 8 A_{-} C$ & 3.0475 & H3BA & .H10BB_m & 2.5844 \\
\hline $\mathrm{H} 4 \mathrm{AA}$ & . C7A_d & 2.8848 & $\mathrm{H} 10 \mathrm{~A}$ & $.01 B$ & 2.7358 \\
\hline H5AA & $.02 \mathrm{~A}$ & 2.3541 & $\mathrm{H} 10 \mathrm{~A}$ &. $\mathrm{H} 8 \mathrm{AB}$ & 2.5774 \\
\hline H5AA & $.02 \_d$ & 2.6231 & $\mathrm{H} 10 \mathrm{~A}$ & .H1OA_i & 2.3658 \\
\hline H8AA & $.02 A_{-} e$ & 2.8315 & $\mathrm{H} 10 \mathrm{~B}$ & $.01 B$ & 2.8572 \\
\hline H8AA & . $\mathrm{C} 2 \mathrm{~B} \_\mathrm{i}$ & 2.9787 & $\mathrm{H} 10 \mathrm{~B}$ & . $\mathrm{C} 2 \mathrm{~A} \mathrm{k}$ & 3.0673 \\
\hline H8AA & . H2BA i & 2.4306 & $\mathrm{H} 10 \mathrm{~B}$ &.$C 3 A \mathrm{k}$ & 2.8149 \\
\hline $\mathrm{H} 8 \mathrm{AB}$ & . H10A & 2.5774 & $\mathrm{H} 4 \mathrm{BA}$ & $.01 \mathrm{f}$ & 2.5694 \\
\hline H9AA &.$C 7 A$ & 2.9569 & H5BA & $.02 \mathrm{~B}$ & 2.3699 \\
\hline $\mathrm{H} 8 \mathrm{C}$ & $.02 B \_g$ & 2.9176 & H5BA & $.01 A_{f} \mathrm{f}$ & 2.6952 \\
\hline $\mathrm{H} 8 \mathrm{C}$ & . C5B_g & 2.8142 & H10BA & $.01 \mathrm{~A}$ & 2.7389 \\
\hline H8D & . H10BA & 2.5482 & H10BA &. $\mathrm{H} 8 \mathrm{D}$ & 2.5482 \\
\hline $\mathrm{H} 8 \mathrm{D}$ & . $\mathrm{C} 2 \mathrm{~B} \_\mathrm{a}$ & 3.0112 & H10BA & . H3BA_b & 2.4932 \\
\hline H8D & . C3B_a & 2.8282 & H10BA & . C5B_a & 2.8143 \\
\hline $\mathrm{H} 2 \mathrm{BA}$ & . C8A i & 3.0754 & $\mathrm{H} 10 \mathrm{BB}$ & $.01 \mathrm{~A}$ & 2.8522 \\
\hline $\mathrm{H} 2 \mathrm{BA}$ & .H8AA_i & 2.4306 & $\mathrm{H} 10 \mathrm{BB}$ & .H3BA_b & 2.5844 \\
\hline H3 BA & $.01 A_{m}$ & 2.7951 & $\mathrm{H} 10 \mathrm{BB}$ & . H2A_k & 2.4337 \\
\hline H3 BA & . $\mathrm{C} 1 \mathrm{~A}$ m & 2.8670 & H9BA &. $\mathrm{C} 7 \mathrm{~B}$ & 2.9301 \\
\hline
\end{tabular}


Table s9 - Hydrogen Bonds (Angstrom, Deg) for: 9

\begin{tabular}{cccccccc}
$\mathrm{C} 5 \mathrm{~A}-\mathrm{H} 5 \mathrm{AA} \ldots \mathrm{O} 2 \mathrm{~A}$ & 0.9300 & 2.3500 & $2.681(6)$ & 100.00 \\
$\mathrm{C} 4 \mathrm{~B}-\mathrm{H} 4 \mathrm{BA} \ldots \mathrm{O}$ & 0.9300 & 2.5700 & $3.413(8)$ & 151.00 & $2-645$ \\
& \multicolumn{2}{c}{$-20-$} &
\end{tabular}

Translation of Symmetry Code to Equiv.Pos

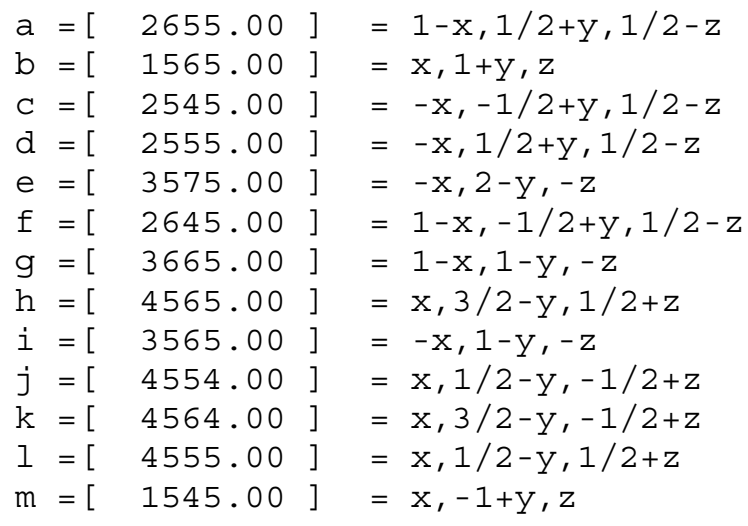

